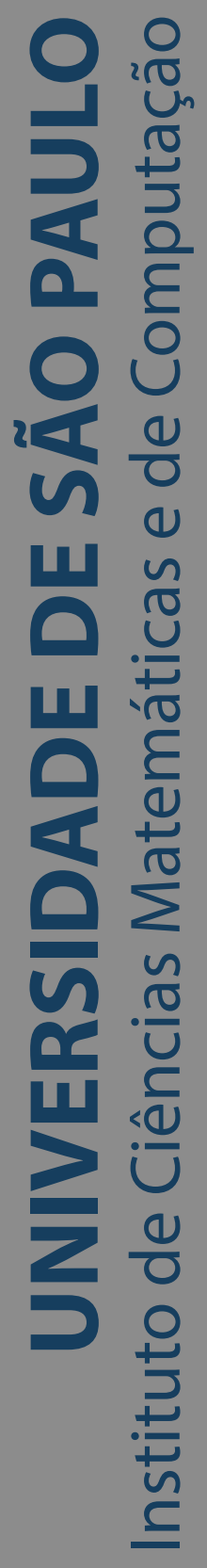

\title{
Periodic solutions of measure and neutral functional
} differential equations

\section{Márcia Richtielle da Silva}

Tese de Doutorado do Programa de Pós-Graduação em Matemática (PPG-Mat) 

SERVIÇO DE PÓS-GRADUAÇÃO DO ICMC-USP

Data de Depósito:

Assinatura:

\section{Márcia Richtielle da Silva}

\section{Periodic solutions of measure and neutral functional differential equations}

\footnotetext{
Thesis submitted to the Instituto de Ciências Matemáticas e de Computação - ICMC-USP - in accordance with the requirements of the Mathematics Graduate Program, for the degree of Doctor in Science. FINAL VERSION
}

Concentration Area: Mathematics

Advisor: Prof. Dr. Everaldo de Mello Bonotto Co-advisor: Profa. Dra. Suzete Maria Silva Afonso 
Ficha catalográfica elaborada pela Biblioteca Prof. Achille Bassi e Seção Técnica de Informática, ICMC/USP, com os dados inseridos pelo(a) autor(a)

Silva, Márcia Richtielle da
P58p Periodic solutions of measure and neutral functional differential equations / Márcia Richtielle da Silva; orientador Everaldo de Mello Bonotto; coorientador Suzete Maria Silva Afonso. -São Carlos, 2021.

$114 \mathrm{p}$.

Tese (Doutorado - Programa de Pós-Graduação em Matemática) -- Instituto de Ciências Matemáticas e de Computação, Universidade de São Paulo, 2021.

1. measure functional differential equations. 2 . neutral functional differential equations. 3 . impulsive differential equations. 4. periodic solutions. 5. fixed-point theorems. I. Bonotto, Everaldo de Mello, orient. II. Afonso, Suzete Maria Silva, coorient. III. Título. 


\title{
Márcia Richtielle da Silva
}

\section{Soluções periódicas de equações diferenciais funcionais neutras e em medida}

\author{
Tese apresentada ao Instituto de Ciências \\ Matemáticas e de Computação - ICMC-USP, \\ como parte dos requisitos para obtenção do título \\ de Doutora em Ciências - Matemática. VERSÃO \\ REVISADA \\ Área de Concentração: Matemática \\ Orientador: Prof. Dr. Everaldo de Mello Bonotto \\ Coorientadora: Profa. Dra. Suzete Maria Silva Afonso
}

USP - São Carlos

Novembro de 2021 

This work is dedicated to my mother Lourdes.

I dedicate my deep admiration and gratitude to you! 

Without a shadow of a doubt, this Ph.D thesis is the materialization of a collective dream... Therefore, it has a lot of meaning, not only for me, but also for all those who directly or indirectly took part in this dream.

At first, I thank to energy, to the supreme consciousness of unconditional love which we are immersed. I thank all the divine forces, the sacred Orixás who govern me, all my spiritual family, all guardians, for so many teachings, directions, so much light and so much love.

I thank my dear advisor Everaldo de Mello Bonotto, for his excellent supervision, always very generous and with admirable humility. I am immensely grateful to you for the opportunity to work with you. Without a doubt, this was a very enriching experience for my professional and personal development! Many thanks for all the patience and lightness with which you took this journey. And for the valuable contribution and dedication for this Ph.D thesis to become a reality. I extend my thanks to my dear co-advisor Suzete Afonso who, since my undergraduate studies, always believed in me and supported me in this journey. Many thanks for all the incentive, friendship, and for the valuable contribution to this work. I really don't have words to express my gratitude to you both! It was a great privilege to share this journey with you!

I especially thank my mother, Lourdes de F. da Silva, for always staying by my side, encouraging me and always wishing me the best. You inspire me with your willpower, your empowerment, with that energy of overcoming and joy that excites everyone. I also thank my dear grandmother, Clotildes Maria da Silva, for the affectionate lap that always cradled and supported me with so much love. To both of you, my dears, I thank you for every prayer, for every vibration of light, love and positivity that you have sent me during this journey. To my brother, Mário Renato da S. Álves, I am grateful for all the help and support you have so lovingly made available to me and our family in all these years that I have been away from home. I thank all the family members who cheered, supported and cheered for everything to work out.

To my love, José Maria da Silva, my partner at all times. For all the times he remained patiently by my side, listening to me, encouraging me, cheering me up, making my walk lighter. To you, my eternal gratitude! I love you!

I also want to thank my spiritual leader and friend, Herica Ponce, for the reception, guidance, care and for all the love she always had, at all times, while I was in the doctorate. To my dear friend Anita Oliveira, for her strength, affection, friendship and for all the incredible moments of sharing. I would like to extend my thanks to the entire family at the Centro de 
Umbanda Cabocla Yara. It's a great honor for me to be part of this great family, which does so much good in the lives of so many people, just as it did in my life! I would not have enough strength to continue this doctorate, if I did not the support of this house, its spirituality and of the dear brothers and sisters of saints, who so lovingly welcomed me to São Carlos.

I can't fail to thank the good, old and new friends who were present, even at a distance, transmitting a lot of affection and messages of support. In particular to Fernanda Andrade, Tiago Pacifico, Isadora Machado, Letícia Rocha, Beatriz Litoldo, Maya Onishi, Diego Mesquita, Cristina Sacilotto, Larissa Soares, Lilian Santos, Silvia Flamini, Izuara Beckmann and all the other friends that I had the honor of sharing so many experiences in these last few years, thank you very much!

Finally, I thank to CAPES for the financial support through the process PROEX10537140/D, without which it would be impossible to dedicate myself exclusively to my studies. 
"A dream you dream alone is only is only a dream that we dream alone, but a dream you dream together is reality."

(Raul Seixas) 



\section{RESUMO}

SILVA, M. R. Soluções periódicas de equações diferenciais funcionais neutras e em medida. 2021. 115 p. Tese (Doutorado em Ciências - Matemática) - Instituto de Ciências Matemáticas e de Computação, Universidade de São Paulo, São Carlos - SP, 2021.

Neste trabalho, investigamos a existência e a unicidade de soluções periódicas para duas classes de equações diferenciais funcionais. Primeiramente, consideramos as equações diferenciais funcionais em medida da forma:

$$
x(t)=x(0)+\int_{0}^{t} f\left(s, x_{s}\right) d s+\int_{0}^{t} g\left(s, x_{s}\right) d u(s),
$$

definida para todo $t \in \mathbb{R}$ e com condições adequadas para as funções $f, g$ e $u$. As integrais do lado direito da equação existem nos sentidos de Perron e Perron-Stieltjes, respectivamente. Utilizando o teorema de transversalidade topológica, exibimos condições suficientes para garantir a existência de soluções periódicas para esse tipo de equação. Como consequência dos resultados obtidos, estudamos a existência de soluções periódicas para uma classe de equações diferenciais funcionais com impulsos.

Além disso, apresentamos o estudo de periodicidade para as soluções da seguinte classe de equações diferenciais funcionais neutras:

$$
\frac{d}{d t}\left(x(t)-A\left(t, x_{t}\right)\right)=f\left(t, x_{t}\right)
$$

definidas quase sempre em $\mathbb{R}$ e com condições adequadas para as funções $A$ e $f$. Neste caso, para garantirmos a existência de soluções periódicas, aplicamos uma variação do teorema de ponto fixo para aplicações condensadas. Como consequência, obtemos a existência e a unicidade de soluções periódicas para uma classe de equações diferenciais funcionais neutras com impulsos. Algumas aplicações são apresentadas com a finalidade de assegurar a aplicabilidade da teoria apresentada.

Os resultados apresentados neste trabalho deram origem aos seguintes artigos:

(1) Periodic solutions of measure functional differential equations. Veja (AFONSO; BONOTTO; SILVA, a).

(2) Periodic solutions of neutral functional differential equations. Veja (AFONSO; BONOTTO; SILVA, b).

Palavras-chave: equações diferenciais funcionais em medida, equações diferenciais funcionais neutras, equações diferenciais impulsivas, soluções periódicas, teoremas de ponto fixo. 



\section{ABSTRACT}

SILVA, M. R. Periodic solutions of measure and neutral functional differential equations. 2021. 115 p. Tese (Doutorado em Ciências - Matemática) - Instituto de Ciências Matemáticas e de Computação, Universidade de São Paulo, São Carlos - SP, 2021.

In this work, we investigate the existence and uniqueness of periodic solutions for two classes of functional differential equations. At first, we consider measure functional differential equations of type:

$$
x(t)=x(0)+\int_{0}^{t} f\left(s, x_{s}\right) d s+\int_{0}^{t} g\left(s, x_{s}\right) d u(s),
$$

defined for every $t \in \mathbb{R}$, under suitable assumptions on $f, g$ and $u$. The integrals on the right-hand side of the equation exist in the senses of Perron and Perron-Stieltjes, respectively. Using a topological transversality theorem, we exhibit sufficient conditions to guarantee the existence of periodic solutions for this type of equation. As a consequence of the obtained results, we study the existence and uniqueness of periodic solutions for a class of functional differential equations with impulses.

In addition, we present a periodicity study for the solutions of the following class of neutral functional differential equations:

$$
\frac{d}{d t}\left(x(t)-A\left(t, x_{t}\right)\right)=f\left(t, x_{t}\right),
$$

defined almost everywhere in $\mathbb{R}$, under suitable assumptions on $A$ and $f$. In this case, in order to guarantee the existence of periodic solutions, we apply a fixed-point theorem variation for condensing maps. As a consequence, we obtain the existence of periodic solutions for a class of impulsive neutral functional differential equations.

Some applications are presented to illustrate the theory.

The new results presented in this work gave rise to the following articles:

(1) Periodic solutions of measure functional differential equations. See (AFONSO; BONOTTO; SILVA, a).

(2) Periodic solutions of neutral functional differential equations. See (AFONSO; BONOTTO; SILVA, b).

Keywords: measure functional differential equations, neutral functional differential equations, impulsive differential equations, periodic solutions, fixed-point theorems. 

INTRODUCTION .................... 17

PRELIMINARES . . . . . . . . . . . . . . . . 25

2.1 Topological transversality theory . . . . . . . . . . . . . 25

$2.2 \quad$ A Fixed-point theorem for condensing maps . . . . . . . . . . . 28

$2.3 \quad$ Regulated functions . . . . . . . . . . . . . . . . 31

$2.4 \quad$ The integrals of Perron and Perron-Stieltjes . . . . . . . . . . 33

2.4.1 The Fundamental Theorem of Calculus . . . . . . . . . . . . . . . . . 36

2.4.2 The Mean Value Theorem . . . . . . . . . . . . . . . 40

2.4.3 Final comments . . . . . . . . . . . . . . . . . . 41

3 MEASURE FUNCTIONAL INTEGRAL EQUATIONS . . . . . . 43

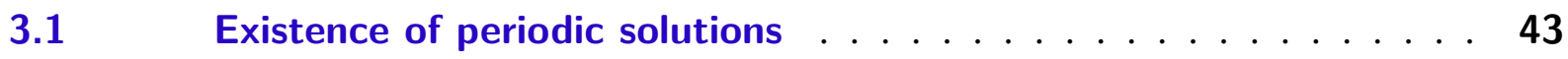

$3.2 \quad$ Priori bounds of the periodic solution $\ldots \ldots \ldots \ldots$

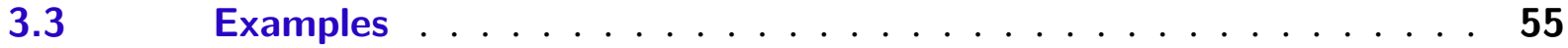

4 IMPULSIVE FUNCTIONAL DIFFERENTIAL EQUATIONS . . . . . 65

$4.1 \quad$ Existence of periodic solutions $\ldots \ldots \ldots \ldots$

$4.2 \quad$ An example . . . . . . . . . . . . . . . . . . 69

5 NEUTRAL FUNCTIONAL DIFFERENTIAL EQUATIONS $\ldots 75$

$5.1 \quad$ Existence of periodic solutions $\ldots \ldots \ldots \ldots$

$5.2 \quad$ Examples ....................... 83

6 NEUTRAL FUNCTIONAL DIFFERENTIAL EQUATIONS WITH IMPULSES ......................... . . 95

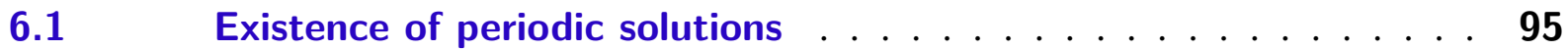

$6.2 \quad$ Examples .......................... 101

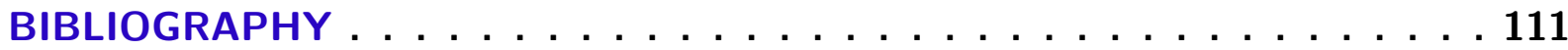



CHAPTER

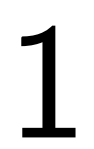

INTRODUCTION

Israelsson and Johnsson proposed in (ISRAELSSON; JOHNSSON, 1967) a study of a model for the geotropic circumnutations of a sunflower. They investigated the helical movements of the growing sunflower tip. The study is based on the lateral growth hormone (auxin) transport, which arises when a gravitational force component acts on the plant. The effect of auxin accumulation in the plant does not occur immediately. There is a time delay between stimulus and response, and then oscillations or circumnutations should appear in the sunflower plant. In (SOMOLINOS, 1978), the author proposes the following equation

$$
x^{\prime \prime}+\frac{a}{r} x^{\prime}+\frac{b}{r} \sin (x(t-r))=0
$$

to describe the movement of the sunflower, where $r$ denotes the geotropic reaction time, $a$ and $b$ are positive constants and $x$ is the angle that the stem makes with a plumb line. This equation is called the "sunflower equation".

Many modeling problems include hereditary effects on the system, that is, the future states of an evolution process depend not only on the present, but also on the past history. An important class of equations that can describe this type of phenomenon is the class of functional differential equations of retarded type. According to J. K. Hale, in the first chapter of the book (ARINO; HBID; DADS, 2002), the theory of functional differential equations have been studied for at least 200 years. Especially in the last 50 years, these equations have been extensively studied by many researchers mainly due to their numerous applications in various areas of science, such as in physical, biological, economic phenomena, among others.

A functional differential equation can be represented by

$$
\dot{x}(t)=f\left(t, x_{t}\right),
$$

where $f:[\sigma-r, \sigma+\omega] \times C\left([-r, 0], \mathbb{R}^{n}\right) \rightarrow \mathbb{R}^{n}$ is a function, $\sigma \in \mathbb{R}, \omega \geq 0, r \geq 0(\omega$ and $r$ may 
be $\infty)$, and for each $t \in[\sigma, \sigma+\omega]$, the map

$$
\begin{aligned}
x_{t}:[-r, 0] & \rightarrow \mathbb{R}^{n} \\
\theta & \mapsto x(t+\theta)
\end{aligned},
$$

called "memory function", is continuous on $[-r, 0]$. The equation (1.1) represents an ordinary differential equation when $r=0$. If $r>0$, the equation (1.1) is known as retarded functional differential equation (RFDE for short). However, when $r=\infty$, equation (1.1) is known as a functional differential equation with infinite delay.

Differential difference equations

$$
\dot{x}(t)=f\left(t, x(t), x\left(t-\tau_{1}(t)\right), \ldots, x\left(t-\tau_{n}(t)\right)\right),
$$

with bounded variable delays $0 \leq \tau_{j}(t) \leq r, j=1, \ldots, n, t \in \mathbb{R}$, are examples of RFDEs. Integrodifferential equations

$$
\dot{x}(t)=\int_{-r}^{0} g(t, s, x(t+s)) d s,
$$

with distributed delay also represent RFDEs. For more details, see (HALE, 1977).

When a functional differential equation involves the derivative of functionals of the past history, this equation is called neutral functional differential equation (neutral FDE for short) and it can be represented by

$$
\dot{x}(t)=f\left(t, x_{t}, \dot{x}_{t}\right) .
$$

The basis of the theory of neutral functional differential equations can be found in (HALE, 1977). These equations cope very well with the description of phenomena in physics, biotechnology, economics and population dynamics. Some qualitative analysis of the periodicity and stability of solutions of neutral functional differential equations were approached in (DIB; MAROUN; RAFFOUL, 2005), (GOPALSAMY, 1992), (GOPALSAMY; HE; WEN, 1991), (HALE, 1977), (ISLAM; RAFFOUL, 2007), (KE, 1990), (LIU; TAKEUCHI, 2007), (STAMOVA; STAMOV, 2016) among others. For illustration purposes, we mention the following neutral FDE

$$
\frac{d}{d t}[x(t)-c x(t-\tau)]=-h(x(t))+h(x(t-\sigma)),
$$

where $h$ is a locally Lipschitz continuous function and increasing, which was investigated in (KRISZTIN; WU, 1996). The neutral FDE (1.4) has been used to model problems of population growth, spread of epidemics, and the dynamics of capital stocks (see (COOKE; KAPLAN, 1976), (COOKE; YORKE, 1973), (BURTON, 2004)).

On the other hand, note that the equivalent integral form of equation (1.1) is given by

$$
x(t)=x(\sigma)+\int_{\sigma}^{t} f\left(s, x_{s}\right) d s, \quad t \in[\sigma, \sigma+\omega] .
$$

A more general form of the integral equation (1.5) is given by

$$
x(t)=x(\sigma)+\int_{\sigma}^{t} f\left(s, x_{s}\right) d g(s), \quad t \in[\sigma, \sigma+\omega],
$$


where the integral on the right-hand side of (1.6) can be considered in the sense of RiemannStieltjes, Lebesgue-Stieltjes or Perron-Stieltjes with respect to a function $g$. The integral equation (1.6) is a generalization of the equation (1.5) and is called a measure functional differential equation (measure FDE for short). The theory of measure differential equations was introduced in (SCHMAEDEKE, 1965) by W. Schmaedeke in the control theory. These equations cope very well with the description of phenomena whose evolution is interrupted by abrupt changes of state, see (DAS; SHARMA, 1971) and (DAS; SHARMA, 1972). It was in (FEDERSON; MESQUITA; SLAVÍK, 2012) that the measure FDE (1.6) was considered with the integral on the right-hand side in the sense of Perron-Stieltjes (or Kurzweil-Henstock-Stieltjes) with respect to a function $g$. The measure FDE (1.6) is an extension of measure differential equations studied consistently, e. g., in (DAS; SHARMA, 1972), (PANDIT; DEO, 1982), (SCHWABIK, 1992), and (SHARMA, 1972).

Motivated by the study of measure FDEs and neutral FDEs and because of their wide applications, we propose in this work a study of the theory of periodic solutions for a class of measure FDEs and a class of neutral FDEs.

In the next lines, we describe the summary of each chapter presented in this work.

Chapter 2 is devoted to present the basic definitions and fundamental results which are used to obtain the main results. In Section 2.1, we present the main tools from the topological transversality theory. In Theorem 2.1.8, we exhibit a variant fixed-point theorem of the nonlinear alternative of Leray-Schauder Principle. In Section 2.2, we state a fixed-point theorem for condensing maps. Section 2.3 concerns the theory of regulated functions. Some auxiliary results are presented and, in Theorem 2.3.7, we exhibit an Arzelá-Ascoli-type theorem for regulated functions taking values in $\mathbb{R}^{n}$. Lastly, in Section 2.4, we describe the theory of integrals in the senses of Perron and Perron-Stieltjes. Sufficient conditions for the existence of a Perron-Stieltjes integral is established in 2.4.5. The change of variable theorem in the context of Perron-Stieltjes is stated in Theorem 2.4.9. Subsection 2.4.1 deals with the Fundamental Theorem of Calculus for Perron integrals. A version of the Fundamental Theorem of Calculus is stated in 2.4.12. A second version is presented in Theorem 2.4.19 and in Corollary 2.4.20. In Subsection 2.4.2, we discuss the Mean Value Theorem for real valued functions that are integrable in the Perron sense, see Theorem 2.4.23. In Subsection 2.4.3, we make some comments on the generality of the Perron integral.

In Chapter 3, we start by presenting our contribution to the field of differential equations. This chapter concerns the study of periodic solutions for measure FDEs of type:

$$
x(t)=x(0)+\int_{0}^{t} f\left(s, x_{s}\right) d s+\int_{0}^{t} g\left(s, x_{s}\right) d u(s), \quad \text { for all } t \in \mathbb{R},
$$

where $f, g: \mathbb{R} \times G_{T}^{-}\left(\mathbb{R}, \mathbb{R}^{n}\right) \rightarrow \mathbb{R}^{n}$ are $T$-periodic functions with respect to the first variable, $T>0$, and $G_{T}^{-}\left(\mathbb{R}, \mathbb{R}^{n}\right)$ denotes the space of all $T$-periodic regulated left-continuous functions $\phi: \mathbb{R} \rightarrow \mathbb{R}^{n}$. As far as we know, there are few results in the literature on periodicity for measure 
differential equations. In (MESQUITA, 2020), the author studied the theory of periodicity for the following measure differential equation

$$
x(t)=x(0)+\int_{0}^{t} b(s) x(s) d g(s), \quad \text { for all } t \in[0, T],
$$

which is a linear measure differential equation. In (AP.SILVA, 2021), the author investigated the existence of periodic solutions for the following measure differential equation

$$
x(t)=x\left(t_{0}\right)+\int_{t_{0}}^{t} f(s, x(s)) d s+\int_{t_{0}}^{t} g(s, x(s)) d u(s), \quad \text { for all } \quad t \in J \subset \mathbb{R} .
$$

Motivated by Jianhong Wu, Huaxing Xia and Bo Zhang in (WU; XIA; ZHANG, 1999), who applied the topological transversality theorem to neutral functional differential equations, by T. A. Burton and Bo Zhang (BURTON; ZHANG., 1991) and by T. A. Burton, P. W. Eloe and M. N. Islam in (BURTON; ISLAM., 1992), who also applied this theory to integrodifferential differential equations with infinite delay, we establish in this work more general conditions to guarantee the existence of periodic solutions for the measure FDE (1.7). Using the nonlinear alternative of Leray-Schauder Principle type, Theorem 2.1.8, we establish in Theorem 3.1.9 sufficient conditions to obtain the existence of $T$-periodic solutions for the measure FDE (1.7). In Theorem 3.1.10, we establish sufficient conditions for the uniqueness of $T$-periodic solutions of the measure FDE (1.7). One of the requirements of the Theorem 3.1.9 is to find a limiting factor for all periodic solutions of a family of measure FDEs

$$
x(t)=\lambda x(0)+\int_{0}^{t} \lambda f\left(s, x_{s}\right) d s+\int_{0}^{t} \lambda g\left(s, x_{s}\right) d u(s), \quad \lambda \in(0,1],
$$

defined for every $t \in \mathbb{R}$. Thus, in Section 3.2, we exhibit in Propositions 3.2.2, 3.2.4, 3.2.5, 3.2.6 and 3.2.7, sufficient conditions to obtain a priori bound for all the periodic solutions of (1.8). In Section 3.3, we present some examples to illustrate the theory. Under additional assumptions, we investigate the existence of periodic solutions for the following equations:

$$
x(t)=x(0)-\int_{0}^{t}\left(\frac{n+\cos ^{2}(n s)}{a_{n}+\sin (n s)}\right) x^{2 m}(s-r) d s+\int_{0}^{t} \sin (s) d u(s)
$$

and

$$
x(t)=x(0)+\int_{0}^{t} a(s) b\left(x\left(s-r_{1}\right)\right) d s+\int_{0}^{t}\left(\int_{-\infty}^{s} K(s, \xi) x(\xi) d \xi\right) d s+\int_{0}^{t} p\left(s, x\left(s-r_{2}\right)\right) d u(s),
$$

see Example 3.3.1 and Example 3.3.2, respectively.

The theory of impulsive differential equations is very important to describe the evolution of systems where the continuous development of a process is interrupted by abrupt changes of state called "impulses". From the point of view of applications, the interest of impulsive differential equations lies in the fact that for many natural phenomena, deterministic models more realistic are often described by equations that involve impulses. There are many applications especially, in the areas of pharmacokinetics, chemical technology, medicine, control areas, finance, 
engineering, physics, biology, among others. See for instance (BACAER; ABDURAHMAN, 2008), (CHIU; JIMÉNEZ, 2010), (DONG; HU; ZHAO, 2018), (KAUFMAN; VENTURA; KASS, 2005), (STAMOVA; STAMOV, 2021) and (FARIA; GADOTTI; OLIVEIRA, 2012).

Due to the importance of the study of impulsive differential equations, we dedicate Chapter 4 to the study of periodic solutions for the following impulsive functional differential equation (impulsive FDE for short) of type

$$
\left\{\begin{array}{l}
\dot{x}(t)=F\left(t, x_{t}\right), \\
\Delta^{+} x\left(t_{k}\right)=I_{k}\left(x\left(t_{k}\right)\right),
\end{array} \quad t \neq t_{k}, \quad k \in \mathbb{Z}\right.
$$

where the moments of impulse effects $\left\{\ldots, t_{-k}, \ldots, t_{-1}, t_{0}, t_{1}, \ldots, t_{k}, \ldots\right\} \subset \mathbb{R}$ satisfy

$$
\ldots<t_{-k}<\ldots<t_{-2}<t_{-1}<t_{0}<t_{1}<t_{2}<\ldots<t_{k}<\ldots
$$

and $\lim _{k \rightarrow \pm \infty} t_{k}= \pm \infty$

It is well known that a function $x$ is a solution of the impulsive FDE (1.9) if and only if $x$ satisfies the following integral equation

$$
x(t)=x(0)+\int_{0}^{t} F\left(s, x_{s}\right) d s+\Gamma(t), \quad t \in \mathbb{R},
$$

where

$$
\Gamma(t)= \begin{cases}\sum_{0<t_{k}<t} I_{k}\left(x\left(t_{k}\right)\right), & \text { if } t \geq 0 \\ -\sum_{t \leq t_{k}<0} I_{k}\left(x\left(t_{k}\right)\right), & \text { if } t<0 .\end{cases}
$$

In Lemma 4.1.2, we prove that it is possible to obtain a correspondence result between the solutions of the integral equation (1.10) and the solutions of a corresponding measure FDE. Through this correspondence result, we establish in Theorem 4.1.5 sufficient conditions for the existence of $T$-periodic solutions of the impulsive FDE (1.9). In Section 4.2, we study the existence of periodic solutions for an impulsive nonlinear Volterra integrodifferential equation with delay of type

$$
\left\{\begin{array}{l}
\dot{x}(t)=a(t) h(x(t))+\int_{t-r}^{t} B(t, s) g(x(s)) d s+p(t), \quad t \neq t_{k}, k \in \mathbb{Z}, \\
x\left(t_{k}^{+}\right)-x\left(t_{k}\right)=b_{k}
\end{array}\right.
$$

under suitable assumptions on $a, h, B, g$ and $p$.

In Section 5, we develop a theory of periodicity for solutions of the following class of NFDEs:

$$
\frac{d}{d t}\left(x(t)-A\left(t, x_{t}\right)\right)=f\left(t, x_{t}\right),
$$

almost everywhere in $\mathbb{R}$, where $A, f: \mathbb{R} \times C_{T}\left(\mathbb{R}, \mathbb{R}^{n}\right) \rightarrow \mathbb{R}^{n}$ are $T$-periodic functions with respect to the first variable, where $C_{T}\left(\mathbb{R}, \mathbb{R}^{n}\right)$ denotes the space of all $T$-periodic continuous functions defined on $\mathbb{R}$ and taking values in $\mathbb{R}^{n}$. In general, the classes of NFDEs presented in the literature 
consider the neutral term $A$ such that, the map $t \mapsto A(t, \cdot)$ is continuous on $[0, T]$ and differentiable on $\mathbb{R}$. Further, it is also considered that the function $f$ is Lipschtiz. In this work, we consider a more general case, we assume that the map $t \mapsto A(t, \cdot)$ has a finite number of discontinuities on $[0, T]$ and $t \mapsto A\left(t, x_{t}\right)$ is differentiable almost everywhere on $\mathbb{R}$, whenever $x \in C_{T}\left(\mathbb{R}, \mathbb{R}^{n}\right)$ is differentiable almost everywhere on $\mathbb{R}$. Moreover, we assume that the mapping $t \mapsto f\left(t, x_{t}\right)$ is locally Perron integrable over $\mathbb{R}$ for every $x \in C_{T}\left(\mathbb{R}, \mathbb{R}^{n}\right)$. Under these conditions, we exhibit in Theorem 5.1.9 sufficient conditions to obtain the existence of $T$-periodic solutions for NFDEs of type (1.12). This result is achieved by using a fixed-point theorem for condensing maps established by D. O'regan, see Theorem 2.2.8. Theorem 5.1.10 concerns the uniqueness of a $T$-periodic solution of the NFDE (1.12). We end this chapter, with Section 5.2, by presenting some examples inspired by (ISLAM; RAFFOUL, 2007), (KE, 1990), and (WU; XIA; ZHANG, 1999) to show the effectiveness of the obtained results. We investigate the existence of periodic solutions for the following neutral delay differential equation

$$
\frac{d}{d t}(x(t)-q(t) x(t-r))=a(t) x(t)+b(t) x(t-r)+p(t),
$$

presented in Example 5.2.1, for the perturbed Van Der Pol equation

$$
z^{\prime \prime}-\varepsilon_{2}\left(1-z^{2}\right) z^{\prime}+z-\varepsilon_{1} \frac{d}{d t} g(t, z(t-r))=0,
$$

presented in Example 5.2.2 and for the neutral Volterra integro-differential equation with infinite delay

$$
\frac{d}{d t}\left[x(t)-\int_{-\infty}^{t} D(t, s) x(s) d s\right]=a(t) x^{k}(t)+\int_{-\infty}^{t} C(t, s) x(s) d s+p(t),
$$

presented in Example 5.2.3.

Lastly, in Chapter 6, we apply the results obtained in Chapter 5 to establish the existence and uniqueness of periodic solutions for a class of impulsive NFDEs of type

$$
\left\{\begin{array}{l}
\frac{d}{d t}\left(x(t)-B\left(t, x_{t}\right)\right)=g\left(t, x_{t}\right), \quad t \neq t_{k}, \quad k \in \mathbb{Z}, \\
x\left(t_{k}^{+}\right)-x\left(t_{k}\right)=b_{k} x\left(t_{k}\right), \quad k \in \mathbb{Z},
\end{array}\right.
$$

where $B, g: \mathbb{R} \times \mathscr{F}\left(\mathbb{R}, \mathbb{R}^{n}\right) \rightarrow \mathbb{R}^{n}$ are $T$-periodic functions with respect to the first variable, the moments of impulse effects satisfy $\ldots<t_{-k}<\ldots<t_{-2}<t_{-1}<0<t_{1}<\ldots<t_{k}<\ldots$, $\lim _{k \rightarrow \pm \infty} t_{k}= \pm \infty$, and $\left\{b_{k}\right\}_{k \in \mathbb{Z}} \subset(-1, \infty), k \in \mathbb{Z}$. The space $\mathscr{F}\left(\mathbb{R}, \mathbb{R}^{n}\right)$ denotes the set of all functions $x: \mathbb{R} \rightarrow \mathbb{R}^{n}$ such that $x \in C\left(\left(t_{j}, t_{j+1}\right], \mathbb{R}^{n}\right)$ and the lateral limit $x\left(t_{j}^{+}\right)$exists for all $j \in \mathbb{Z}$.

In Section 6.1, motivated by the techniques developed in (LI; KOU; DUAN, 2009), (YAN; ZHAO, 1998), (ZHAO; FENG; DING, 2015) and (FURTADO, 2012), we obtain sufficient conditions to guarantee the existence and uniqueness of periodic solutions for the impulsive system (1.13), making use of a non-impulsive equation conveniently associated with this impulsive problem. See Theorems 6.1.8 and 6.1.9. 
In Section 6.2, we present some examples. In Example 6.2.1, we study the existence of periodic solutions for the Lasota-Wazewska equation type

$$
\left\{\begin{array}{l}
\frac{d}{d t}(x(t)-C(t) x(t-r))=-A(t) x(t)+\sum_{j=1}^{n} d_{j}(t) e^{-\gamma_{j}(t) x_{j}(t-\tau)}, t \neq t_{k}, k \in \mathbb{Z}, \\
x\left(t_{k}^{+}\right)-x\left(t_{k}\right)=\frac{2}{\left(4+(-1)^{k+1}+1\right)} \cos (k \pi) x\left(t_{k}\right), \quad k \in \mathbb{Z},
\end{array}\right.
$$

and in Example 6.2.2, we study the existence of periodic solutions to the following impulsive neural networks differential equation with infinity delay

$$
\left\{\begin{array}{l}
\frac{d}{d t}(x(t)-P(t) x(t-r(t)))=-C(t) x(t-\sigma(t))+A(t) h(x(t-\tau(t))) \\
+\int_{-\infty}^{t} K(t-s) g(x(s)) d s+J(t), \quad t \neq t_{k}, \quad k \in \mathbb{Z} \\
x\left(t_{k}^{+}\right)-x\left(t_{k}\right)=b_{k} x\left(t_{k}\right), \quad k \in \mathbb{Z} .
\end{array}\right.
$$



CHAPTER

In this chapter, we present the fundamental tools that we will make use to obtain the main results. In Section 2.1, we recall some concepts from the topological transversality theory. Section 2.2 concerns with a version of a fixed-point theorem of Krasnoselskii type. In Section 2.3, we exhibit some results from the theory of regulated functions. Lastly, in Section 2.4, we present the concepts and basic properties of the integrals of Perron and Perron-Stieltjes.

\subsection{Topological transversality theory}

This section deals with some important tools from the theory of topological transversality. We shall present some basic definitions and results which will be useful to develop the theory presented in the next chapters. The reader may consult (GRANAS; DUGUNDJI, 2013) to obtain more details.

Let $E$ be a Banach space and $Y \subset E$ be a convex subset. Let $X \subset Y$ be a subset and $A \subset X$ be a subset such that $A$ is closed in $X$.

Definition 2.1.1. A map $T: Y \subseteq E \rightarrow E$ is said to be completely continuous if it is continuous on $Y$ and compact, that is, $T$ maps any bounded subset of $Y$ into a relatively compact subset of $E$.

Definition 2.1.2. A map $F: X \rightarrow Y$ has a fixed point on $A$ if there is $x \in A$ such that $F(x)=x$. The map $F$ is fixed point free on $A$ if $F(x) \neq x$ for all $x \in A$.

We denote by $\mathscr{K}_{A}(X, Y)$ the set of all completely continuous maps from $X$ to $Y$ which are fixed point free on $A$.

Definition 2.1.3. A map $F \in \mathscr{K}_{A}(X, Y)$ is said to be essencial when every map $G \in \mathscr{K}_{A}(X, Y)$ that satisfies $\left.F\right|_{A}=\left.G\right|_{A}$ has a fixed point in $X$. 
Example 2.1.4. Let $X \subset Y$ be a closed subset and $A=\partial X$ (the boundary of $X$ in $Y$ ). For each fixed $p \in X \backslash A$, the constant map

$$
\begin{aligned}
f: X & \rightarrow Y \\
x & \mapsto p
\end{aligned}
$$

is an essential map in $\mathscr{K}_{A}(X, Y)$. In fact, let $G \in \mathscr{K}_{A}(X, Y)$ be such that $\left.f\right|_{A}=\left.G\right|_{A}$. In order to show that $G$ has a fixed point, let $G^{*}: Y \rightarrow Y$ be an extension of the function $G$, given by

$$
G^{*}(x)= \begin{cases}G(x), & x \in X, \\ p, & x \in Y \backslash X .\end{cases}
$$

By construction, $G^{*}$ is a continuous map. Besides that, since $\overline{G^{*}(Y)}=\overline{G(X) \cup\{p\}}=\overline{G(X)} \cup\{p\}$ and $G$ is a compact map, it follows that $G^{*}$ is a compact map. By Schauder's Fixed Point Theorem, $G^{*}$ has a fixed point $x^{*} \in Y$. Since $f$ has no fixed point on $A$, we conclude that $x^{*} \in X \backslash A$. Thus,

$$
x^{*}=G^{*}\left(x^{*}\right)=G\left(x^{*}\right),
$$

that is, $x^{*}$ is a fixed poinf of $G$. Therefore, $f$ is an essential map in $\mathscr{K}_{A}(X, Y)$.

According to Definition 2.1.3, every essential map admits at least one fixed point in $X \backslash A$. In this way, it is interesting to determine when a map in $\mathscr{K}_{A}(X, Y)$ is essential. Sufficient and necessary conditions can be established via homotopy.

Definition 2.1.5. Two maps $F$ and $G$ in $\mathscr{K}_{A}(X, Y)$ are called homotopic in $\mathscr{K}_{A}(X, Y)$, if there exists a completely continuous map $H:[0,1] \times X \rightarrow Y$ such that $H_{\lambda}:=H(\lambda, \cdot): X \rightarrow Y$ belongs to $\mathscr{K}_{A}(X, Y)$ for each $\lambda \in[0,1], H_{0}=F$ and $H_{1}=G$. In this case, we say that $F$ and $G$ are homotopic by the homotopy $H$.

Theorem 2.1.6. (GRANAS; DUGUNDJI, 2013, Lemma 4.6) Let $F \in \mathscr{K}_{A}(X, Y)$. Then the following conditions are equivalent:

(i) $F$ is not essential;

(ii) there is $G \in \mathscr{K}_{A}(X, Y)$ which is fixed point free on $X$ such that $F$ and $G$ are homotopic in $\mathscr{K}_{A}(X, Y)$

(iii) there is $F^{*} \in \mathscr{K}_{A}(X, Y)$ which is fixed point free on $X$ such that $F$ and $F^{*}$ are homotopic in $\mathscr{K}_{A}(X, Y)$ by a homotopy $H^{*}$ satisfying $\left.H^{*}\right|_{[0,1] \times A}=\left.F\right|_{A}$.

Proof. $(i) \Rightarrow($ ii $)$ Since $F$ is not essential, there exists a map $G \in \mathscr{K}_{A}(X, Y)$ which is fixed point free on $X$, with $\left.F\right|_{A}=\left.G\right|_{A}$. Now, we define $H:[0,1] \times X \rightarrow Y$ by

$$
H(\lambda, x)=\lambda G(x)+(1-\lambda) F(x),
$$

which is a completely continuous homotopy between $F$ and $G$. Since

$$
H(\lambda, x)=\lambda G(x)+(1-\lambda) F(x)=\lambda F(x)+(1-\lambda) F(x)=F(x) \neq x
$$


for all $x \in A$, we have $H(\lambda, \cdot) \in \mathscr{K}_{A}(X, Y)$ for each $\lambda \in[0,1]$.

$($ ii $) \Rightarrow($ iii $)$ Let $H:[0,1] \times X \rightarrow Y$ be a completely continuous homotopy between $G$ and $F$ such that $H(0, x)=G(x), H(1, x)=F(x)$, and $H(\lambda, \cdot)$ is fixed point free on $A$ for each $\lambda \in[0,1]$. We have two cases to consider:

Case 1) $H(\lambda, x) \neq x$ for all $\lambda \in[0,1]$ and for all $x \in X$.

In this case, consider the following constant map defined on $A$

$$
\begin{aligned}
f: A & \rightarrow \mathbb{R} \\
x & \mapsto 1
\end{aligned}
$$

Since $A$ is closed in $X$, we may use the Tietze's Theorem to find a continuous extension $\alpha: X \rightarrow[0,1]$ of $f$. Define $F^{*}(x)=H(\alpha(x), x), x \in X$. Note that $F^{*}$ is a completely continuous map and it is also fixed point free on $X$.

Now, let us show that $F^{*}$ and $F$ are homotopic in $\mathscr{K}_{A}(X, Y)$. Indeed, consider the completely continuous homotopic map $H^{*}:[0,1] \times X \rightarrow Y$ given by

$$
H^{*}(\lambda, x)=H((1-\lambda)+\lambda \alpha(x), x) \text {. }
$$

Then $H^{*}(0, x)=H(1, x)=F(x)$ and $H^{*}(1, x)=H(\alpha(x), x)=F^{*}(x)$, for all $x \in X$. Since $\alpha(x)=$ 1 for all $x \in A$, we have

$$
H^{*}(\lambda, x)=H(1, x)=F(x) \neq x,
$$

for all $\lambda \in[0,1]$ and $x \in A$. Hence, $H^{*}(\lambda, \cdot) \in \mathscr{K}_{A}(X, Y)$ for each $\lambda \in[0,1]$. Furthermore, the relation (2.1) implies that $\left.H^{*}\right|_{[0,1] \times A}=\left.F\right|_{A}$.

Case 2) There is $x_{0} \in X$ such that $H\left(\lambda, x_{0}\right)=x_{0}$ for some $\lambda \in[0,1]$.

Consider the set

$$
B=\{x \in X: H(\lambda, x)=x \text {, for some } \lambda \in[0,1]\}
$$

Note that $B$ is a nonempty closed subset of $X$ since $H(\lambda, \cdot)$ is continuous on $X$. Besides that, $A \cap B=\emptyset$ because $H(\lambda, \cdot)$ is fixed point free in $A$ for every $\lambda \in[0,1]$. Hence, there is an Urysohn function $\alpha: X \rightarrow[0,1]$ with

$$
\left.\alpha\right|_{A}=1 \quad \text { and }\left.\quad \alpha\right|_{B}=0 .
$$

Define $F^{*}: X \rightarrow Y$ by $F^{*}(x)=H(\alpha(x), x), x \in X$, which is a completely continuous map and it is also a fixed point free on $X$. Indeed, if $F^{*}(x)=H(\alpha(x), x)=x$ then $x \in B$. Thus, $\alpha(x)=0$ which implies $x=H(0, x)=G(x)$ and this contradicts the assumption that $G$ is fixed point free on $X$.

Now, let us show that $F^{*}$ and $F$ are homotopic in $\mathscr{K}_{A}(X, Y)$. For this, consider the completely continuous homotopic

$$
H^{*}(\lambda, x)=H((1-\lambda)+\lambda \alpha(x), x), \quad \lambda \in[0,1], x \in X
$$


Note that $H^{*}(0, x)=H(1, x)=F(x)$ and $H^{*}(1, x)=H(\alpha(x), x)=F^{*}(x)$, for all $x \in X$. Since $\alpha(x)=1$ for all $x \in A$, we conclude that

$$
H^{*}(\lambda, x)=H(1, x)=F(x) \neq x
$$

for all $\lambda \in[0,1]$ and $x \in A$. Thus, $H^{*}(\lambda, \cdot) \in \mathscr{K}_{A}(X, Y)$ for each $\lambda \in[0,1]$, and $\left.H^{*}\right|_{[0,1] \times A}=\left.F\right|_{A}$.

$(i i i) \Rightarrow(i)$ By hypothesis there is $F^{*} \in \mathscr{K}_{A}(X, Y)$, with $F^{*}(x) \neq x$ for all $x \in X$, and a homotopy $H^{*}(\lambda, \cdot) \in \mathscr{K}_{A}(X, Y)$, for each $\lambda \in[0,1]$, such that $H_{0}^{*}=F, H_{1}^{*}=F^{*}$ and $\left.H^{*}\right|_{[0,1] \times A}=$ $\left.F\right|_{A}$. Hence, we have $\left.F^{*}\right|_{A}=\left.H_{1}^{*}\right|_{A}=\left.F\right|_{A}$ with $F^{*}(x) \neq x$, for all $x \in X$. This implies that $F$ is not an essential map.

Theorem 2.1.7 gives a characterization of essential maps via homotopy. The proof is an immediate consequence of Theorem 2.1.6.

Theorem 2.1.7. (GRANAS; DUGUNDJI, 2013, Theorem 4.7) Let $F$ and $G$ be homotopic in $\mathscr{K}_{A}(X, Y)$. Then $F$ is essential if and only if $G$ is essential.

We end this section by presenting a consequence of Theorem 2.1.7, which is a variant of the nonlinear alternative of Leray-Schauder Principle (see Theorems 5.1 and 5.2 in (GRANAS; DUGUNDJI, 2013)).

Theorem 2.1.8. (WU; XIA; ZHANG, 1999, Theorem 2.2) Let $X \subset Y$ be closed, $p \in X \backslash \partial X$ and $N: X \rightarrow Y$ be a completely continuous map. If $H:[0,1] \times X \rightarrow Y$ is a completely continuous homotopy such that $H_{0} \equiv p$ and $H_{1} \equiv N$, then either

(i) $N$ has a fixed point in $X \backslash \partial X$; or

(ii) there are $x \in \partial X$ and $\lambda \in(0,1]$ such that $x=H(\lambda, x)$.

Proof. Assume that (i) does not hold. If $N$ has a fixed point in $\partial X$, then (ii) holds for $\lambda=1$ and we get the result. Otherwise, $N$ is fixed point free on $X$. In this case, if (ii) does not hold, then $H(\lambda, \cdot) \in \mathscr{K}_{\partial X}(X, Y)$ for all $\lambda \in[0,1]$. Since $H_{0} \equiv p$ and $H_{1} \equiv N$ are homotopic in $\mathscr{K}_{\partial X}(X, Y)$ and the constant map $X \ni x \mapsto p$ is essential in $\mathscr{K}_{\partial X}(X, Y)$ (see Example 2.1.4), it follows from Theorem 2.1.7 that $N$ is essential. Therefore, $N$ must have a fixed point in $X$. But this is a contradiction. Therefore, condition (ii) holds.

\subsection{A Fixed-point theorem for condensing maps}

This section concerns some important tools from the fixed-point theory for the sum of two operators, established by D. O'regan in (O'REGAN, 1996). As stated in (O'REGAN, 1996), this is a variation of the fixed-point theorem (nonlinear alternative for condensing maps) that 
extends a theorem of Krasnoselskii. The reader may consult (ZEIDLER, 1986, Theorem 11.B) for more details.

Let $E$ be a Banach space and $\Omega_{E}$ be a family of bounded subsets of $E$.

Definition 2.2.1. The Kuratowski measure of non-compactness is a map

$$
\alpha: \Omega_{E} \rightarrow[0, \infty)
$$

defined by

$$
\alpha(X)=\inf \left\{\varepsilon>0: X \subseteq \bigcup_{i=1}^{n} X_{i} \text { and } \operatorname{diam}\left(X_{i}\right)<\varepsilon\right\}
$$

for each $X$ in $\Omega_{E}$.

Let's recall some properties of the Kuratowski measure of non-compactness. Given $A, B \in \Omega_{E}$, we have:

(i) $\alpha(A)=0$ if and only if $\bar{A}$ is compact;

(ii) if $A \subseteq B$ then $\alpha(A) \leq \alpha(B)$;

(iii) $\alpha(A \cup B)=\max \{\alpha(A), \alpha(B)\}$;

(iv) $\alpha(r A)=|r| \alpha(A)$, for $r \in \mathbb{R}$;

(v) $\alpha(A+B) \leq \alpha(A)+\alpha(B)$.

The proofs of these properties and other details can be found in (ZEIDLER, 1986, Chapter 11).

Definition 2.2.2. Let $Y \subset E$ be a subset. A set $U$ is called relatively open in $Y$, if there is an open set $V$ in $E$ such that $U=V \cap Y$.

Let $E_{1}$ and $E_{2}$ be two Banach spaces and let $F: Y \subseteq E_{1} \rightarrow E_{2}$ be a continuous map which maps bounded sets into bounded sets.

Definition 2.2.3. The map $F$ is called $\alpha$-Lipschitzian if there is a constant $k \geq 0$ such that

$$
\alpha(F(X)) \leq k \alpha(X), \quad \text { for all bounded set } \quad X \subseteq Y \text {. }
$$

Definition 2.2.4. The map $F$ is called condensing if $\alpha(F(X))<\alpha(X)$ for all bounded subset $X \subseteq Y$ with $\alpha(X) \neq 0$.

Remark 2.2.5. Note that if $F$ is $\alpha$-Lipschitzian with $0 \leq k<1$ then, $F$ is a condensing map. Moreover, every compact map is also a $\alpha$-Lipschitz map with $k=0$. Hence, every compact map is a condensing map. 
Definition 2.2.6. The map $F$ is called nonlinear contraction, if there exists a continuous nondecreasing function $\phi:[0, \infty) \rightarrow[0, \infty)$ satisfying $\phi(z)<z$ for all $z>0$, such that

$$
\|F(x)-F(y)\| \leq \phi(\|x-y\|), \quad \text { for all } \quad x, y \in Y .
$$

The next theorem gives us a nonlinear alternative for condensing maps.

Theorem 2.2.7. (GRANAS; DUGUNDJI, 2013, Chapter 6-subsection 9-(C.7))(AGARWAL; MEEHAN; O'REGAN, 2001, Theorem 5.6) Let $E$ be a Banach space and $C \subseteq E$ be a closed and convex subset. Assume that $U$ is a relatively open subset of $C$ with $0 \in U, F(\bar{U})$ is bounded and $F: \bar{U} \rightarrow C$ is a condensing continuous map. Then either,

(i) $F$ has a fixed point in $\bar{U}$; or

(ii) there are $u \in \partial U$ and $\lambda \in(0,1)$ such that $u=\lambda F(u)$.

A special case of Theorem 2.2.7 is presented in the next result.

Theorem 2.2.8. (O'REGAN, 1996, Theorem 2.1) Let $E$ be a Banach space and $C \subset E$ be a closed and convex subset. Let $U$ be an open set in $C$. Assume that $0 \in U, F(\bar{U})$ is bounded and $F: \bar{U} \rightarrow C$ is given by $F=F_{1}+F_{2}$, where $F_{1}: \bar{U} \rightarrow C$ is a completely continuous map and $F_{2}: \bar{U} \rightarrow C$ is a nonlinear contraction map. Then either,

(i) $F$ has a fixed point in $\bar{U}$; or

(ii) there are $u \in \partial U$ and $\lambda \in(0,1)$ such that $u=\lambda F(u)$.

Proof. The result follows as a consequence of Theorem 2.2.7. In fact, it is enough to show that $F: \bar{U} \rightarrow C$ is a condensing map. Let $A$ be a bounded subset of $\bar{U}$ with $\alpha(A)>0$. Then,

$$
\alpha(F(A)) \leq \alpha\left(F_{1}(A)\right)+\alpha\left(F_{2}(A)\right)=\alpha\left(F_{2}(A)\right),
$$

since $F_{1}$ maps bounded sets into relatively compact sets (and, hence, $\alpha\left(F_{1}(A)\right)=0$ ). Now, we claim that

$$
\alpha\left(F_{2}(A)\right) \leq \phi(\alpha(A))
$$

holds, where $\phi$ is the continuous nondecreasing function that comes from nonlinear contraction condition of $F_{2}$. Indeed, given $\varepsilon>0$, there are subsets $A_{1}, \ldots, A_{n}$ in $E$ such that

$$
A \subseteq \bigcup_{i=1}^{n} A_{i} \quad \text { and } \quad \operatorname{diam}\left(A_{i}\right)<\alpha(A)+\varepsilon
$$

for each $i=1, \ldots, n$. Note that

$$
F_{2}(A) \subseteq \bigcup_{i=1}^{n} F_{2}\left(A_{i}\right)
$$


Let $i_{0} \in\{1, \ldots, n\}$ be fixed but arbitrary. If $w_{0}, w_{1} \in F_{2}\left(A_{i_{0}}\right)$, then there exist $x_{0}, x_{1} \in A_{i_{0}}$ such that $F_{2}\left(x_{0}\right)=w_{0}$ and $F_{2}\left(x_{1}\right)=w_{1}$. Since $\phi$ is nondecreasing and $F_{2}$ is a nonlinear contraction map, we obtain

$$
\left\|w_{0}-w_{1}\right\|=\left\|F_{2}\left(x_{0}\right)-F_{2}\left(x_{1}\right)\right\| \leq \phi\left(\left\|x_{0}-x_{1}\right\|\right) \leq \phi(\alpha(A)+\varepsilon) .
$$

Consequently, $\operatorname{diam}\left(F\left(A_{i}\right)\right) \leq \phi(\alpha(A)+\varepsilon)$ for every $i=1, \ldots, n$, as $i_{0}$ was taken arbitrary. From the definition of the Kuratowski measure of non-compactness, we get

$$
\alpha\left(F_{2}(A)\right) \leq \phi(\alpha(A)+\varepsilon)
$$

By the arbitrariness of $\varepsilon>0$, the inequality (2.3) holds. Therefore, by (2.2), (2.3) and the property of $\phi$, we have

$$
\alpha(F(A)) \leq \phi(\alpha(A))<\alpha(A),
$$

which proves that $F$ is a condensing map. The proof is complete.

\subsection{Regulated functions}

In this section, we present the concept of regulated functions and some of its properties.

Let $E$ be a Banach space endowed with a norm $\|\cdot\|$ and $a, b \in \mathbb{R}$, with $a<b$.

Definition 2.3.1. A function $f:[a, b] \rightarrow E$ is called regulated if the lateral limits,

$$
f\left(t^{-}\right)=\lim _{s \rightarrow t^{-}} f(s), \quad t \in(a, b], \quad \text { and } \quad f\left(t^{+}\right)=\lim _{s \rightarrow t^{+}} f(s), \quad t \in[a, b),
$$

exist. The set of all regulated functions $f:[a, b] \rightarrow E$ is denoted by $G([a, b], E)$.

Theorem 2.3.2. (HÖNIG, 1975, Theorem I.3.6) The space $G([a, b], E)$ equipped with the usual supremum norm $|f|_{\infty}=\sup _{t \in[a, b]}\|f(t)\|$ is a Banach space.

The space of all regulated functions which are left-continuous is denoted by $G^{-}([a, b], E)$. Since $G^{-}([a, b], E)$ is a closed subspace of $G([a, b], E)$, the space $G^{-}([a, b], E)$ is also Banach when equipped with the supremum norm $|\cdot|_{\infty}$. By $G^{-}(\mathbb{R}, E)$, we denote the space of all functions $f: \mathbb{R} \rightarrow E$ such that the restriction $\left.f\right|_{[a, b]}$ belongs to $G^{-}([a, b], E)$, with $a<b$.

Let $T>0$. The vector space of all $T$-periodic left-continuous regulated functions is represented by

$$
G_{T}^{-}(\mathbb{R}, E)=\left\{f \in G^{-}(\mathbb{R}, E): f \text { is } T \text {-periodic }\right\}
$$

and it is a Banach space with the norm $|f|_{T}=\sup _{t \in[0, T]}\|f(t)\|$.

In what follows, we present some properties of equiregulated sets. Equiregulated sets are very important to characterize compact sets in $G\left([a, b], \mathbb{R}^{n}\right)$, see Theorems 2.3.6 and 2.3.7. 
Definition 2.3.3. A subset $\mathscr{A} \subset G([a, b], E)$ is called equiregulated if, for every $\varepsilon>0$ and every $\sigma \in[a, b]$, there exists $\delta>0$ such that:

(i) if $x \in \mathscr{A}, t^{\prime} \in[a, b]$ and $\sigma-\delta<t^{\prime}<\sigma$, then $\left\|x\left(\sigma^{-}\right)-x\left(t^{\prime}\right)\right\|<\varepsilon$;

(ii) if $x \in \mathscr{A}, t^{\prime \prime} \in[a, b]$ and $\sigma<t^{\prime \prime}<\sigma+\delta$, then $\left\|x\left(t^{\prime \prime}\right)-x\left(\sigma^{+}\right)\right\|<\varepsilon$.

The next theorem gives us a characterization of the class of equiregulated functions. This result was first established by D. Franková in the case that $E=\mathbb{R}^{n}$, see (FRANKOVÁ, 2019, Proposition 3.2).

Theorem 2.3.4. (BONOTTO; FEDERSON; MESQUITA, 2021, Theorem 1.11) A subset $\mathscr{A} \subset$ $G([a, b], E)$ is equiregulated if and only if for every $\varepsilon>0$, there exists a division $a=t_{0}<t_{1}<$ $\ldots<t_{n}=b$ of $[a, b]$ such that

$$
\left\|x\left(t^{\prime}\right)-x(t)\right\| \leq \varepsilon
$$

for every $x \in \mathscr{A}$ and $\left[t, t^{\prime}\right] \subset\left(t_{j-1}, t_{j}\right)$, for $j=1,2, \ldots, n$.

Theorem 2.3.5 describes a property of equiregulated sets $\mathscr{A}$ of $G([a, b], E)$. This result was first established by D. Franková in (FRANKOVÁ, 1991, Lemma 2.2) when $E=\mathbb{R}^{n}$.

Theorem 2.3.5. (BONOTTO; FEDERSON; MESQUITA, 2021, Theorem 1.12) Assume that a set $\mathscr{A} \subset G([a, b], E)$ is equiregulated and, for all $t \in[a, b]$, there is a number $\gamma_{t}$ such that

$$
\left\|x(t)-x\left(t^{-}\right)\right\| \leq \gamma_{t}, \quad t \in(a, b] \quad \text { and } \quad\left\|x\left(t^{+}\right)-x(t)\right\| \leq \gamma_{t}, \quad t \in[a, b) .
$$

Then there is $K>0$ such that, for every $x \in \mathscr{A}$

$$
\|x(t)-x(a)\| \leq K, \quad t \in[a, b] .
$$

When $E=\mathbb{R}^{n}$, it is possible to characterize compact sets in $G\left([a, b], \mathbb{R}^{n}\right)$. Theorem 2.3.6 describes necessary and sufficient conditions for a subset of $G\left([a, b], \mathbb{R}^{n}\right)$ to be relatively compact.

Theorem 2.3.6. (FRANKOVÁ, 1991, Proposition 2.3) A subset $\mathscr{A} \subset G\left([a, b], \mathbb{R}^{n}\right)$ is relatively compact if and only if it is equiregulated and, for all $t \in[a, b]$, there is a number $\gamma_{t}$ such that

$$
\left\|x(t)-x\left(t^{-}\right)\right\| \leq \gamma_{t} \text { for } t \in(a, b], \quad\left\|x\left(t^{+}\right)-x(t)\right\| \leq \gamma_{t} \text { for } t \in[a, b),
$$

and there exists $\alpha>0$ such that $\|x(a)\| \leq \alpha$, for any $x \in \mathscr{A}$.

Theorem 2.3.7 is an Arzelá-Ascoli-type theorem for regulated functions on $[a, b]$ taking values in $\mathbb{R}^{n}$.

Theorem 2.3.7. (FRANKOVÁ, 2019, Corollary 4.3) A set $\mathscr{A} \subset G\left([a, b], \mathbb{R}^{n}\right)$ is relatively compact in $G\left([a, b], \mathbb{R}^{n}\right)$ if and only if $\mathscr{A}$ is equiregulated and, for every $t \in[a, b]$, the set $\{x(t): x \in \mathscr{A}\}$ is bounded. 
Proof. If $\mathscr{A}$ is relatively compact, then by Theorem 2.3.6, $\mathscr{A}$ is equiregulated, condition (2.6) holds and $\|x(a)\| \leq \alpha$ for all $x \in \mathscr{A}$. Moreover, by Theorem 2.3.5, there is $K>0$ such that

$$
\|x(t)-x(a)\| \leq K
$$

for all $x \in \mathscr{A}$ and all $t \in[a, b]$. Thus,

$$
\|x(t)\| \leq\|x(t)-x(a)\|+\|x(a)\| \leq K+\alpha
$$

for all $x \in \mathscr{A}$ and all $t \in[a, b]$. Therefore, $\{x(t): x \in \mathscr{A}\}$ is bounded.

Reciprocally, assume that $\mathscr{A}$ is equiregulated and, for each $t \in[a, b]$, there exists $\beta_{t}>0$ such that $\|x(t)\| \leq \beta_{t}$ for all $x \in \mathscr{A}$. Let $t \in(a, b)$ be given. Since $\mathscr{A}$ is equiregulated, there is $\delta>0$ such that, for each $x \in \mathscr{A}$, we have

$$
\begin{array}{cc}
\left\|x(\tau)-x\left(b^{-}\right)\right\| \leq 1, & \tau \in(b-\delta, b), \\
\left\|x(\tau)-x\left(t^{-}\right)\right\| \leq 1, & \tau \in(t-\delta, t), \\
\left\|x\left(a^{+}\right)-x(\tau)\right\| \leq 1, & \tau \in(a, a+\delta), \\
\left\|x\left(t^{+}\right)-x(\tau)\right\| \leq 1, & \tau \in(t, t+\delta) .
\end{array}
$$

Let $\tau_{1} \in(t-\delta, t), \tau_{2} \in(t, t+\delta), \tau_{b} \in(b-\delta, b)$ and $\tau_{a} \in(a, a+\delta)$ be fixed. Then

$$
\begin{gathered}
\left\|x(t)-x\left(t^{-}\right)\right\| \leq\|x(t)\|+\left\|x\left(\tau_{1}\right)\right\|+\left\|x\left(\tau_{1}\right)-x\left(t^{-}\right)\right\| \leq \beta_{t}+\beta_{\tau_{1}}+1, \\
\left\|x\left(t^{+}\right)-x(t)\right\| \leq\left\|x\left(t^{+}\right)-x\left(\tau_{2}\right)\right\|+\left\|x\left(\tau_{2}\right)\right\|+\|x(t)\| \leq 1+\beta_{\tau_{2}}+\beta_{t}, \\
\left\|x(b)-x\left(b^{-}\right)\right\| \leq\|x(b)\|+\left\|x\left(\tau_{b}\right)\right\|+\left\|x\left(\tau_{b}\right)-x\left(b^{-}\right)\right\| \leq \beta_{b}+\beta_{\tau_{b}}+1,
\end{gathered}
$$

and

$$
\left\|x\left(a^{+}\right)-x(a)\right\| \leq\left\|x\left(a^{+}\right)-x\left(\tau_{a}\right)\right\|+\left\|x\left(\tau_{a}\right)\right\|+\|x(a)\| \leq 1+\beta_{\tau_{a}}+\beta_{a} .
$$

Set $\gamma_{a}=1+\beta_{\tau_{a}}+\beta_{a}, \gamma_{b}=\beta_{b}+\beta_{\tau_{b}}+1$ and $\gamma_{t}=1+\beta_{t}+\max \left\{\beta_{\tau_{1}}, \beta_{\tau_{2}}\right\}$, for $t \in(a, b)$. In conclusion, $\|x(a)\| \leq \beta_{a}$ for all $x \in \mathscr{A}$ and condition (2.6) holds. Hence, by Theorem 2.3.6, $\mathscr{A}$ is relatively compact.

\subsection{The integrals of Perron and Perron-Stieltjes}

In this section, we present basic concepts of integration in the senses of Perron and PerronStieltjes. For more details, the reader may consult the references (BARTLE, 2001; BONOTTO; FEDERSON; MESQUITA, 2021; HENSTOCK, 1991; SCHWABIK, 1992; SCHWABIK, 1996; YEE et al., 2000).

Definition 2.4.1. A tagged division of a compact interval $[a, b] \subset \mathbb{R}$ is a finite collection of point-interval pairs $d=\left\{\left(\tau_{i},\left[s_{i-1}, s_{i}\right]\right)\right\}_{i=1}^{|d|}$, where $a=s_{0} \leq s_{1} \leq \ldots \leq s_{|d|}=b$ is a division of $[a, b], \tau_{i} \in\left[s_{i-1}, s_{i}\right]$, and $|d|$ denotes the number of intervals in which $d$ is divided. 
Definition 2.4.2. A gauge on $[a, b]$ is any strictly positive function $\delta:[a, b] \rightarrow(0, \infty)$.

Definition 2.4.3. Given a gauge $\delta:[a, b] \rightarrow(0, \infty)$, we say that a tagged division

$$
d=\left\{\left(\tau_{i},\left[s_{i-1}, s_{i}\right]\right)\right\}_{i=1}^{|d|}
$$

is $\delta$-fine whenever $\left[s_{i-1}, s_{i}\right] \subset\left(\tau_{i}-\delta\left(\tau_{i}\right), \tau_{i}+\delta\left(\tau_{i}\right)\right)$ for every $i=1,2, \ldots,|d|$.

Let $E$ be a Banach space endowed with a norm $\|\cdot\|$ and $a, b \in \mathbb{R}$, with $a<b$. Now, we can state the concept of the Perron-Stieltjes integral for functions defined on $[a, b]$ and taking values in $E$.

Definition 2.4.4. A function $f:[a, b] \rightarrow E$ is called Perron-Stieltjes integrable over $[a, b]$ with respect to a function $u:[a, b] \rightarrow \mathbb{R}$ if there is $L \in E$ such that, for all $\varepsilon>0$, there exists a gauge $\delta:[a, b] \rightarrow(0, \infty)$ such that whenever $d=\left\{\left(\tau_{i},\left[s_{i-1}, s_{i}\right]\right)\right\}_{i=1}^{|d|}$ is a $\delta$-fine tagged division of $[a, b]$, then

$$
\left\|\sum_{i=1}^{|d|} f\left(\tau_{i}\right)\left(u\left(s_{i}\right)-u\left(s_{i-1}\right)\right)-L\right\|<\varepsilon .
$$

In this case, we denote $\int_{a}^{b} f(s) d u(s)=L$.

The Perron-Stieltjes integral satisfies the standard properties as linearity, additivity over nonoverlapping intervals, etc. See (BONOTTO; FEDERSON; MESQUITA, 2021) for more details.

Theorem 2.4.5 provides us sufficient conditions for the existence of a Perron-Stieltjes integral and, moreover, it shows that the indefinite integral is regulated. This result is a consequence of (SCHWABIK, 1992, Corollary 1.34) and (SCHWABIK, 1992, Theorem 1.16) for the $n$-dimensional case.

Theorem 2.4.5. (BONOTTO; FEDERSON; MESQUITA, 2021, Corollary 2.10) Let $f:[a, b] \rightarrow$ $E$ and $g:[a, b] \rightarrow \mathbb{R}$ be functions such that $f$ is regulated on $[a, b]$ and $g$ is of bounded variation in $[a, b]$. Then, the Perron-Stieltjes integral $\int_{a}^{b} f(t) d g(t)$ exists. Moreover, the functions

$$
h(t)=\int_{a}^{t} f(s) d g(s) \text { and } k(t)=\int_{t}^{b} f(s) d g(s)
$$

are regulated on $[a, b]$ and satisfy

$$
\begin{aligned}
& h\left(t^{+}\right)=h(t)+f(t) \Delta^{+} g(t) \quad \text { and } \quad k\left(t^{+}\right)=k(t)-f(t) \Delta^{+} g(t), \quad t \in[a, b) \text {, } \\
& h\left(t^{-}\right)=h(t)-f(t) \Delta^{-} g(t) \quad \text { and } \quad k\left(t^{-}\right)=k(t)+f(t) \Delta^{-} g(t), \quad t \in(a, b],
\end{aligned}
$$

where $\Delta^{+} g(t)=g\left(t^{+}\right)-g(t)$ and $\Delta^{-} g(t)=g(t)-g\left(t^{-}\right)$.

Remark 2.4.6. If $u(s)=s, s \in[a, b]$, in Definition 2.4.4, then the function $f:[a, b] \rightarrow E$ is said to be Perron integrable over $[a, b]$. The Perron integral is equivalent to the integral of Kurzweil-Henstock for functions taking values in a finite dimensional space, see (YEE et al., 2000, Theorem 3.1.3). 
Remark 2.4.7. As presented in (BARTLE, 2001; YEE et al., 2000), the Perron-Stieltjes (Perron) integral can be extended to unbounded intervals.

In what follows, we present some properties of the Perron-Stieltjes and Perron integrals that we will make use in the development of this work.

Theorem 2.4.8 exhibits sufficient conditions to estimate the value of a Perron integral. A proof of it can be found in (SCHWABIK, 1992, Theorem 1.35) for the $n$-dimensional case. For functions taking values in Banach spaces, a proof of Theorem 2.4.8 can be obtained as a consequence of (BONOTTO; FEDERSON; MESQUITA, 2021, Theorem 2.15).

Theorem 2.4.8. Let $f:[a, b] \rightarrow E$ be Perron integrable over $[a, b]$. If $V:[a, b] \rightarrow \mathbb{R}$ is Perron integrable over $[a, b]$ and if there exists a gauge $\theta$ on $[a, b]$ such that

$$
|t-\tau|\|f(\tau) t-f(\tau) \tau\| \leq(t-\tau)[V(\tau) t-V(\tau) \tau],
$$

para todo $t \in(\tau-\theta(\tau), \tau+\theta(\tau))$, then the inequality

$$
\left\|\int_{a}^{b} f(s) d s\right\| \leq \int_{a}^{b} V(s) d s
$$

holds.

Next, we exhibit a change of variable theorem for the Perron-Stieltjes integral. This result is a consequence of (BONOTTO; FEDERSON; MESQUITA, 2021, Theorem 2.18).

Theorem 2.4.9. Let $-\infty<c<d<\infty$. Assume that $\phi:[c, d] \rightarrow \mathbb{R}$ is a continuous strictly monotone function. Let $g:[\phi(c), \phi(d)] \rightarrow E$ and $u:[\phi(c), \phi(d)] \rightarrow \mathbb{R}$ be given functions. If one of the integrals $\int_{\phi(c)}^{\phi(d)} g(s) d u(s)$ or $\int_{c}^{d} g(\phi(s)) d u(\phi(s))$ exists, then the other also exists and the equality

$$
\int_{\phi(c)}^{\phi(d)} g(s) d u(s)=\int_{c}^{d} g(\phi(s)) d u(\phi(s))
$$

holds.

In Lemma 2.4.10, we present an auxiliary result which will be essential in Chapter 4, to exhibit a correspondence between an impulsive FDE and its associated measure FDE.

Lemma 2.4.10. (BONOTTO; FEDERSON; MESQUITA, 2021, Lemma 3.5) Let $a \leq r_{1}<$ $r_{2}<\ldots<r_{m} \leq b$, with $m \in \mathbb{N}$. Consider a pair of functions $g:[a, b] \rightarrow E$ and $u:[a, b] \rightarrow \mathbb{R}$, where $u$ is regulated, left-continuous on $[a, b]$ and continuous at $r_{1}, \ldots, r_{m}$. Let $\tilde{g}:[a, b] \rightarrow E$ and $\tilde{u}:[a, b] \rightarrow \mathbb{R}$ be such that $\tilde{g}(t)=g(t)$ for every $t \in[a, b] \backslash\left\{r_{1}, \ldots, r_{m}\right\}$ and $\tilde{u}-u$ is constant on each of the intervals $\left[a, r_{1}\right],\left(r_{1}, r_{2}\right], \ldots,\left(r_{m-1}, r_{m}\right],\left(r_{m}, b\right]$. Then, the Perron-Stieltjes integral $\int_{a}^{b} \tilde{g}(s) d \tilde{u}(s)$ exists, if and only if the Perron-Stieltjes integral $\int_{a}^{b} g(s) d u(s)$ exists. In this case, we have

$$
\int_{a}^{b} \tilde{g}(s) d \tilde{u}(s)=\int_{a}^{b} g(s) d u(s)+\sum_{\substack{k \in\{1, \ldots, m\} \\ r_{k}<b}} \tilde{g}\left(r_{k}\right) \Delta^{+} \tilde{u}\left(r_{k}\right)
$$


where $\Delta^{+} \tilde{u}\left(r_{k}\right)=\tilde{u}\left(r_{k}^{+}\right)-\tilde{u}\left(r_{k}\right), k=1,2, \ldots, m$.

\subsubsection{The Fundamental Theorem of Calculus}

This subsection deals with the Fundamental Theorem of Calculus for Perron integrals. We start by presenting an auxiliary result called the Straddle Lemma.

Lemma 2.4.11. (Straddle) (BONOTTO; FEDERSON; MESQUITA, 2021, Lemma 1.86) Let $f:[a, b] \rightarrow E$ be differentiable at a point $t \in[a, b]$. Given $\varepsilon>0$, there is $\delta_{\varepsilon}(t)>0$ such that if $u, v \in[a, b]$ satisfy

$$
t-\delta_{\varepsilon}(t) \leq u \leq t \leq v \leq t+\delta_{\varepsilon}(t)
$$

then

$$
\left\|f(v)-f(u)-f^{\prime}(t)(v-u)\right\| \leq \varepsilon(v-u) .
$$

In Theorem 2.4.12, we present a version of the Fundamental Theorem of Calculus for Perron integrals. This result is concerned with the integration of derivatives. Its proof is based on the proof for the one-dimensional case found in (BARTLE, 2001, Section 4, Theorems 4.7 and 4.11). The reader also may consult (YEE et al., 2000) for the case that $E=\mathbb{R}^{n}$.

Theorem 2.4.12. If $f:[a, b] \rightarrow E$ is continuous on $[a, b]$ and differentiable almost everywhere on $[a, b]$, then $f^{\prime}$ is Perron integrable over $[a, b]$ and

$$
\int_{a}^{b} f^{\prime}(s) d s=f(b)-f(a)
$$

Proof. Let $C=\left\{c_{k}\right\}_{k \in \mathbb{N}}$ be the subset of $[a, b]$, with measure equals to zero, such that $f$ is differentiable on $[a, b] \backslash C$. In this case, we can consider $f^{\prime}\left(c_{k}\right)=0$ for every $k \in \mathbb{N}$ (otherwise, we may consider a function $g: \mathbb{R} \rightarrow E$ such that $g(s)=f(s)$ for all $s \in[a, b], g^{\prime}(s)=f^{\prime}(s)$ for every $s \in[a, b] \backslash C$, and $g^{\prime}\left(c_{k}\right)=0$ for every $k \in \mathbb{N}$, consequently, $g^{\prime}$ is Perron integrable over $[a, b]$ and $\left.\int_{a}^{b} g^{\prime}(s) d s=\int_{a}^{b} f^{\prime}(s) d s\right)$.

The next step is to define a gauge on $[a, b]$. If $t \in[a, b] \backslash C$, by using the Straddle Lemma, given $\varepsilon>0$, let $\delta_{\varepsilon}(t)$ be as in Lemma 2.4.11. On the other hand, if $t \in C$ then $t=c_{k}$ for some $k \in \mathbb{N}$. Since $f$ is continuous on $[a, b]$, we can choose $\delta_{\varepsilon}\left(c_{k}\right)>0$ such that if $\left|x-c_{k}\right| \leq \delta_{\varepsilon}\left(c_{k}\right)$ then

$$
\left\|f(x)-f\left(c_{k}\right)\right\| \leq \frac{\varepsilon}{2^{k+2}} .
$$

Hence, a gauge on $[a, b]$ is given by

$$
\delta(t)= \begin{cases}\delta_{\varepsilon}(t), & \text { if } t \in[a, b] \backslash C, \\ \delta_{\mathcal{\varepsilon}}\left(c_{k}\right), & \text { if } t=c_{k}, k \in \mathbb{N} .\end{cases}
$$

Let $d=\left\{\left(\tau_{i},\left[s_{i-1}, s_{i}\right]\right)\right\}_{i=1}^{|d|}$ be a $\delta$-fine tagged division of $[a, b]$. Note that if $\tau_{i} \in[a, b] \backslash C$ for some $i \in\{1, \ldots,|d|\}$ then, by Lemma 2.4.11, we get

$$
\left\|f\left(s_{i}\right)-f\left(s_{i-1}\right)-f^{\prime}\left(\tau_{i}\right)\left(s_{i}-s_{i-1}\right)\right\| \leq \frac{\varepsilon}{2(b-a)}\left(s_{i}-s_{i-1}\right)
$$


since $\left[s_{i-1}, s_{i}\right] \subset\left(\tau_{i}-\delta\left(\tau_{i}\right), \tau_{i}+\delta\left(\tau_{i}\right)\right)$. Consequently, using (2.8), we obtain

$$
\sum_{\tau_{i} \in[a, b] \backslash C}\left\|f\left(s_{i}\right)-f\left(s_{i-1}\right)-f^{\prime}\left(\tau_{i}\right)\left(s_{i}-s_{i-1}\right)\right\| \leq \sum_{i=1}^{|d|} \frac{\varepsilon}{2(b-a)}\left(s_{i}-s_{i-1}\right)=\frac{\varepsilon}{2} .
$$

Now, if $\tau_{i}=c_{k}$, for some $k$, then using (2.7), we have

$$
\begin{aligned}
\| f\left(s_{i}\right)-f\left(s_{i-1}\right) & -f^{\prime}\left(c_{k}\right)\left(s_{i}-s_{i-1}\right) \| \leq \\
& \leq\left\|f\left(s_{i}\right)-f\left(c_{k}\right)\right\|+\left\|f\left(c_{k}\right)-f\left(s_{i-1}\right)\right\|+\left\|f^{\prime}\left(c_{k}\right)\left(s_{i}-s_{i-1}\right)\right\| \\
& \leq \frac{\varepsilon}{2^{k+2}}+\frac{\varepsilon}{2^{k+2}}+0=\frac{\varepsilon}{2^{k+1}},
\end{aligned}
$$

once $\left[s_{i-1}, s_{i}\right] \subset\left(\tau_{i}-\delta\left(\tau_{i}\right), \tau_{i}+\delta\left(\tau_{i}\right)\right)$, for all $i \in\{1, \ldots,|d|\}$. Hence,

$$
\sum_{\tau_{i} \in C}\left\|f\left(s_{i}\right)-f\left(s_{i-1}\right)-f^{\prime}\left(\tau_{i}\right)\left(s_{i}-s_{i-1}\right)\right\| \leq \sum_{k=1}^{|d|} \frac{\varepsilon}{2^{k+1}} \leq \sum_{k=1}^{\infty} \frac{\varepsilon}{2^{k+1}}=\frac{\varepsilon}{2}
$$

Thus, using the fact that $f(b)-f(a)=\sum_{i=1}^{|d|}\left[f\left(s_{i}\right)-f\left(s_{i-1}\right)\right],(2.9)$ and (2.10), we obtain

$$
\begin{aligned}
& \left\|f(b)-f(a)-\sum_{i=1}^{|d|} f^{\prime}\left(\tau_{i}\right)\left(s_{i}-s_{i-1}\right)\right\|= \\
& =\left\|\sum_{i=1}^{|d|}\left[f\left(s_{i}\right)-f\left(s_{i-1}\right)\right]-\sum_{i=1}^{|d|} f^{\prime}\left(\tau_{i}\right)\left(s_{i}-s_{i-1}\right)\right\| \\
& \leq \sum_{i=1}^{|d|}\left\|f\left(s_{i}\right)-f\left(s_{i-1}\right)-f^{\prime}\left(\tau_{i}\right)\left(s_{i}-s_{i-1}\right)\right\| \\
& =\sum_{\tau_{i} \in[a, b] \backslash C}\left\|f\left(s_{i}\right)-f\left(s_{i-1}\right)-f^{\prime}\left(\tau_{i}\right)\left(s_{i}-s_{i-1}\right)\right\| \\
& \quad+\sum_{\tau_{i} \in C}\left\|f\left(s_{i}\right)-f\left(s_{i-1}\right)-f^{\prime}\left(\tau_{i}\right)\left(s_{i}-s_{i-1}\right)\right\| \\
& \leq \frac{\varepsilon}{2}+\frac{\varepsilon}{2}=\varepsilon,
\end{aligned}
$$

whenever $d=\left\{\left(\tau_{i},\left[s_{i-1}, s_{i}\right]\right)\right\}_{i=1}^{|d|}$ is a $\delta$-fine tagged division of $[a, b]$. Therefore, by the arbitrariness of $\varepsilon>0$, we conclude the desired result.

According to Theorem 2.4.5, we can state the following result.

Theorem 2.4.13. If $f:[a, b] \rightarrow E$ is Perron integrable over $[a, b]$ and $F(x)=\int_{a}^{x} f(s) d s, a \leq x \leq b$, then $F \in C([a, b], E)$.

Another version of the Fundamental Theorem of Calculus says that if $F$ is an indefinite integral of $f$ on $[a, b]$, then $F$ is almost everywhere differentiable on $[a, b]$, and $F^{\prime}(x)=f(x)$ for almost everywhere on $[a, b]$. However, this result is known for functions taking values in finite dimensional spaces. 
In what follows, we prove this second version of the Fundamental Theorem of Calculus for functions taking values in $\mathbb{R}^{n}$. In order to do that, we state the Saks-Henstock Lemma (that holds for general Banach spaces) and the Vitali Covering Theorem which will be useful to prove Theorem 2.4.20.

Lemma 2.4.14. (Saks-Henstock) (BONOTTO; FEDERSON; MESQUITA, 2021, Lemma 1.45) Let $f:[a, b] \rightarrow E$ be a Perron-Stieltjes integrable function over $[a, b]$ with respect to a function $u:[a, b] \rightarrow \mathbb{R}$. Given $\varepsilon>0$, let $\delta:[a, b] \rightarrow(0, \infty)$ be a gauge on $[a, b]$ such that for every $\delta$-fine tagged division $d=\left\{\left(\tau_{i},\left[s_{i-1}, s_{i}\right]\right)\right\}_{i=1}^{|d|}$ of $[a, b]$,

$$
\left\|\mid \sum_{i=1}^{|d|} f\left(\tau_{i}\right)\left(u\left(s_{i}\right)-u\left(s_{i-1}\right)\right)-\int_{a}^{b} f(s) d u(s)\right\|<\varepsilon .
$$

Then, if $a \leq \alpha_{1} \leq \xi_{1} \leq \beta_{1} \leq \alpha_{2} \leq \xi_{2} \leq \beta_{2} \leq \ldots \leq \alpha_{p} \leq \xi_{p} \leq \beta_{p}=b$ represents a $\delta$-fine tagged division of $[a, b]$ then

$$
\left\|\sum_{i=1}^{p}\left[f\left(\xi_{i}\right)\left(u\left(\alpha_{i}\right)-u\left(\beta_{i}\right)\right)-\int_{\alpha_{i}}^{\beta_{i}} f(s) d u(s)\right]\right\| \leq \varepsilon .
$$

Corollary 2.4.15. With the hypotheses of Lemma 2.4.14, if $f:[a, b] \rightarrow \mathbb{R}$ is a real valued function and $a \leq \alpha_{1} \leq \xi_{1} \leq \beta_{1} \leq \alpha_{2} \leq \xi_{2} \leq \beta_{2} \leq \ldots \leq \alpha_{p} \leq \xi_{p} \leq \beta_{p}=b$ represents a $\delta$-fine tagged division of $[a, b]$ then

$$
\sum_{i=1}^{p}\left|f\left(\xi_{i}\right)\left(u\left(\alpha_{i}\right)-u\left(\beta_{i}\right)\right)-\int_{\alpha_{i}}^{\beta_{i}} f(s) d u(s)\right| \leq 2 \varepsilon .
$$

Definition 2.4.16. Let $I \subseteq[a, b]$ and $\mathscr{F}$ be a collection of nondegenerate closed subintervals in $[a-1, b+1]$. The collection $\mathscr{F}$ is called a Vitali covering for $I$ if, for every $x \in I$ and every $\varepsilon>0$ there is an interval $J \in \mathscr{F}$ such that $x \in J$ and $0<\ell(J)<\varepsilon$, where $\ell(J)$ denotes the length of the interval $J$.

Example 2.4.17. A countable Vitali covering for $I=[0,1]$ is the collection of all closed balls $\mathscr{F}=\left\{\left[r-\frac{1}{n}, r+\frac{1}{n}\right]: r \in I \cap \mathbb{Q}, n \in \mathbb{N}\right\}$. Indeed, for every $x \in I$ and every $\varepsilon>0$, there is $N \in \mathbb{N}$ such that $\frac{2}{\varepsilon}<N$. Then, taking $J=\left[x-\frac{1}{N}, x+\frac{1}{N}\right]$, we obtain $x \in J$ and $\ell\left(\left[x-\frac{1}{N}, x+\frac{1}{N}\right]\right)=\frac{2}{N}<\varepsilon$.

Theorem 2.4.18. (Vitali Covering Theorem) (BARTLE, 2001, Theorem 5.8) Let $I \subseteq[a, b]$ and let $\mathscr{F}$ be a Vitali covering for $I$. Then, given $\varepsilon>0$ there exist disjoint intervals $I_{1}, \ldots, I_{p}$ from $\mathscr{F}$ and a countable collection of closed intervals $J_{p+1}, J_{p+2}, \ldots$ in $\mathbb{R}$ such that

$$
I-\bigcup_{i=1}^{p} I_{i} \subseteq \bigcup_{i=p+1}^{\infty} J_{i} \text { and } \sum_{i=p+1}^{\infty} \ell\left(J_{i}\right) \leq \varepsilon .
$$

Therefore, it follows that

$$
I \subseteq\left(\bigcup_{i=1}^{p} I_{i}\right) \cup\left(\bigcup_{i=p+1}^{\infty} J_{i}\right)
$$


In Theorem 2.4.19, we consider the second version of the Fundamental Theorem of Calculus for real valued functions.

Theorem 2.4.19. (BARTLE, 2001, Theorem 5.9) If $f:[a, b] \rightarrow \mathbb{R}$ is Perron integrable over $[a, b]$ and $F(x)=\int_{a}^{x} f(s) d s, a \leq x \leq b$, then $F$ is almost everywhere differentiable on $[a, b]$, and $F^{\prime}(x)=f(x)$ for almost everywhere on $[a, b]$.

Proof. We are going to show that there is a set of null measure $C \subset[a, b]$ such that $F^{\prime}(x)=f(x)$, for all $x \in[a, b] \backslash C$. Indeed, let $C_{1}$ denote the set of all points in $x \in[a, b)$ such that the right hand derivative $F_{+}^{\prime}(x)$ either does not exist at $x$ or $F_{+}^{\prime}(x) \neq f(x)$. Analogously, let $C_{2}$ denote the set of all points in $x \in(a, b]$ such that the left hand derivative $F_{-}^{\prime}(x)$ either does not exist at $x$ or $F_{-}^{\prime}(x) \neq f(x)$. Set $C=C_{1} \cup C_{2}$. We claim that $C_{1}$ and $C_{2}$ have null measure. Let us prove that $C_{1}$ has null measure as the proof of $C_{2}$ is analogous. In fact, if the right hand derivative $F_{+}^{\prime}(x)=f(x)$ for some $x \in[a, b)$, then for all $\alpha>0$ there is $\delta_{\alpha, x}>0$ such that if $u \in[a, b]$ and $x<u<x+\delta_{\alpha, x}$, then

$$
\left|\frac{F(u)-F(x)}{u-x}-f(x)\right| \leq \alpha .
$$

Hence, if $x \in C_{1}$ then there is $\alpha(x)>0$ such that, for every $\delta_{1}>0$, one can find $u_{x, \delta_{1}} \in[a, b]$ such that $x<u_{x, \delta_{1}}<x+\delta_{1}$ and

$$
\left|\frac{F\left(u_{x, \delta_{1}}\right)-F(x)}{u_{x, \delta_{1}}-x}-f(x)\right|>\alpha(x),
$$

that is,

$$
\left|F\left(u_{x, \delta_{1}}\right)-F(x)-f(x)\left(u_{x, \delta_{1}}-x\right)\right|>\alpha(x)\left(u_{x, \delta_{1}}-x\right) .
$$

Let $n \in \mathbb{N}$ be fixed and consider the set $I_{n}=\left\{x \in C_{1}: \alpha(x) \geq \frac{1}{n}\right\}$. Since $f$ is Perron integrable over $[a, b]$, given $\varepsilon>0$ there is a gauge $\delta_{\varepsilon}$ on $[a, b]$ such that if $d=\left\{\left(\tau_{i},\left[s_{i-1}, s_{i}\right]\right)\right\}_{i=1}^{|d|}$ is a $\delta_{\varepsilon}$-fine tagged division of $[a, b]$, then

$$
\left|\sum_{i=1}^{|d|} f\left(\tau_{i}\right)\left(x_{i}-x_{i-1}\right)-\int_{a}^{b} f(x) d x\right|<\frac{\varepsilon}{n} .
$$

On the other hand, define a collection

$$
\mathscr{F}_{n}=\left\{\left[x, u_{x, \delta_{1}}\right]: x \in I_{n}, 0<\delta_{1} \leq \delta_{\varepsilon}(x)\right\} .
$$

Note that $\mathscr{F}_{n}$ is a Vitali covering for $I_{n}$, since $\left[x, u_{x, \delta_{1}}\right] \subseteq[a, b] \subset[a-1, b+1]$ for all $x \in I_{n}$, and, for a given $x \in I_{n}$ and $\varepsilon>0$, we can choose $\delta_{1} \in(0, \varepsilon) \cap\left(0, \delta_{\varepsilon}(x)\right]$, which implies that $J=\left[x, u_{x, \delta_{1}}\right] \in \mathscr{F}_{n}, x \in J$ and

$$
\ell(J)=u_{x, \delta_{1}}-x<\delta_{1}<\varepsilon .
$$

Therefore, by Theorem 2.4.18, there are intervals $I_{1}=\left[x_{1}, u_{1}\right], \ldots, I_{p}=\left[x_{p}, u_{p}\right]$ in $\mathscr{F}_{n}$ and closed intervals $J_{p+1}, J_{p+2}, \ldots$ of $\mathbb{R}$ such that

$$
I_{n} \subseteq\left(\bigcup_{i=1}^{p} I_{i}\right) \cup\left(\bigcup_{i=p+1}^{\infty} J_{i}\right) \quad \text { and } \quad \sum_{i=p+1}^{\infty} \ell\left(J_{i}\right) \leq \varepsilon .
$$


Now, by using Corollary 2.4.15, we get

$$
\sum_{i=1}^{p}\left|f\left(x_{i}\right)\left(u_{i}-x_{i}\right)-\left[F\left(u_{i}\right)-F\left(x_{i}\right)\right]\right|=\sum_{i=1}^{p}\left|f\left(x_{i}\right)\left(u_{i}-x_{i}\right)-\int_{x_{i}}^{u_{i}} f(x) d x\right| \leq \frac{2 \varepsilon}{n},
$$

since $\left\{\left(x_{i},\left[x_{i}, u_{i}\right]\right)\right\}_{i=1}^{p}$ is a $\delta_{\varepsilon}$-fine tagged division of $[a, b]$. On the other hand, observe that by (2.11)

$$
\sum_{i=1}^{p}\left|f\left(x_{i}\right)\left(u_{i}-x_{i}\right)-\left[F\left(u_{i}\right)-F\left(x_{i}\right)\right]\right|>\sum_{i=1}^{p} \alpha\left(x_{i}\right)\left(u_{i}-x_{i}\right) \geq \frac{1}{n} \sum_{i=1}^{p}\left(u_{i}-x_{i}\right) .
$$

By (2.13) and (2.14), we conclude that $\frac{1}{n} \sum_{i=1}^{p}\left(u_{i}-x_{i}\right)<\frac{2 \varepsilon}{n}$, that is,

$$
\sum_{i=1}^{p} \ell\left(I_{i}\right)=\sum_{i=1}^{p}\left(u_{i}-x_{i}\right)<2 \varepsilon
$$

Thus, by (2.12), we obtain

$$
\ell\left(I_{n}\right)=\sum_{i=1}^{p} \ell\left(I_{i}\right)+\sum_{i=p+1}^{\infty} \ell\left(J_{i}\right) \leq 3 \varepsilon .
$$

Since $\varepsilon>0$ is arbitrary, it follows that $I_{n}$ is a null set. But, since $C_{1}=\cup_{i=1}^{\infty} I_{i}$, we conclude that $C_{1}$ has null measure.

Analogously, we prove that $C_{2}$ has null measure. Therefore $C=C_{1} \cup C_{2}$ has null measure and $F^{\prime}(x)=f(x)$ for all $[a, b] \backslash C$.

Corollary 2.4.20. If $f:[a, b] \rightarrow \mathbb{R}^{n}$ is Perron integrable over $[a, b]$ and $F(x)=\int_{a}^{x} f(s) d s, a \leq x \leq$ $b$, then $F$ is almost everywhere differentiable on $[a, b]$, and $F^{\prime}(x)=f(x)$ for almost everywhere on $[a, b]$.

Theorem 2.4.21 below is a consequence of (BARTLE, 2001, Corollary 4.9).

Theorem 2.4.21. Let $f:[a, b] \rightarrow \mathbb{R}^{n}$ be Perron integrable over $[a, b]$ and $F(x)=\int_{a}^{x} f(s) d s$, $a \leq x \leq b$. If $f$ is continuous at $c \in[a, b]$ then $F$ is differentiable at $c$ and $F^{\prime}(c)=f(c)$.

\subsubsection{The Mean Value Theorem}

The Mean Value Theorem also holds for Perron integrals as shown in the next result.

Theorem 2.4.22. If $f:[a, b] \rightarrow \mathbb{R}$ is continuous on $[a, b]$ and Perron integrable over $[a, b]$, then there exists $\xi \in(a, b)$ such that $\int_{a}^{b} f(s) d s=f(\xi)(b-a)$.

Proof. Let $F(x)=\int_{a}^{x} f(s) d s$ be defined for $x \in[a, b]$. It follows by Theorem 2.4.21 that $F$ is differentiable on $[a, b]$ and $F^{\prime}(x)=f(x)$ for every $x \in[a, b]$. By the Mean Value Theorem for derivatives there exists $\xi \in(a, b)$ such that $F^{\prime}(\xi)=\frac{F(b)-F(a)}{b-a}$. Then

$$
\frac{1}{b-a} \int_{a}^{b} f(s) d s=\frac{F(b)-F(a)}{b-a}=F^{\prime}(\xi)=f(\xi) .
$$


If $f$ is continuous on $(a, b)$, we also can obtain the Mean Value Theorem for Perron integrals.

Theorem 2.4.23. If $f:[a, b] \rightarrow \mathbb{R}$ is continuous on $(a, b)$ and Perron integrable over $[a, b]$, then there exists $\xi \in(a, b)$ such that

$$
\int_{a}^{b} f(s) d s=f(\xi)(b-a)
$$

Proof. Define $g:[a, b] \rightarrow \mathbb{R}$ by

$$
g(t)= \begin{cases}f\left(a^{+}\right), & \text {if } t=a, \\ f(t), & \text { if } t \in(a, b), \\ f\left(b^{-}\right), & \text {if } t=b .\end{cases}
$$

Thus, $g$ is continuous on $[a, b]$ and Perron integrable over $[a, b]$ by Theorem 2.4.5. Now, according to Theorem 2.4.22, there exists $\xi \in(a, b)$ such that

$$
g(\xi)=\frac{1}{b-a} \int_{a}^{b} g(s) d s .
$$

Since $\int_{a}^{b} f(s) d s=\int_{a}^{b} g(s) d s$ and $g(\xi)=f(\xi)$, we obtain the desired result.

\subsubsection{Final comments}

The Perron integral is more general than the Riemann and the Lebesgue integrals. Several functions that have many discontinuities and/or are of unbounded variation are integrable in the sense of Perron. As presented in (BARTLE, 2001; BONOTTO; FEDERSON; MESQUITA, 2021; YEE et al., 2000), it is known that:

(A) if $f$ is Riemann or Riemann improper integrable then $f$ is Perron integrable;

(B) if $f$ is Lebesgue integrable then $f$ is Perron integrable.

In order to show that the set of Perron integrable functions contains properly the set of Riemann and the set of Lebesgue integrable functions, we exhibit an example of a function that is neither Riemann nor Lesbegue integrable but it is Perron integrable. This illustrates an important point about the theory of Perron integrals that is the non-absolute integrability. For more details, see (KURTZ; SWARTZ, 2004), (BARTLE, 2001).

Example 2.4.24. Let $F:[0,1] \rightarrow \mathbb{R}$ be given by

$$
F(t)= \begin{cases}t^{2} \sin \left(\frac{1}{t^{2}}\right), & \text { if } \quad 0<t \leq 1 \\ 0, & \text { if } t=0\end{cases}
$$


The function $F$ is differentiable on $[0,1]$, with derivative $f:[0,1] \rightarrow \mathbb{R}$ given by

$$
f(t)= \begin{cases}2 t \sin \left(\frac{1}{t}\right)-\cos \left(\frac{1}{t}\right), & \text { if } 0<t \leq 1 \\ 0, & \text { if } t=0\end{cases}
$$

It follows by Theorem 2.4.12 that $f$ is Perron integrable over [0,1], since $F$ is differentiable on $[0,1]$. But, the function $f$ it is not Riemann integrable on $[0,1]$, since $f$ is unbounded on $[0,1]$. Now, let us show that $f$ is not Lebesgue integrable on $[0,1]$. Note that for $a, b \in \mathbb{R}$ with $0<a<b<1, f$ is continuous on $[a, b]$. Then, $f$ is Riemann integrable on $[a, b]$. By the Fundamental Theorem of Calculus for Riemann integrals, we have

$$
\int_{a}^{b} f(s) d s=\int_{a}^{b} F^{\prime}(s) d s=F(b)-F(a)=b^{2} \sin \left(\frac{1}{b^{2}}\right)-a^{2} \sin \left(\frac{1}{a^{2}}\right) .
$$

Setting $a_{k}=\frac{1}{\sqrt{(2 k+1) \pi}}$ and $b_{k}=\sqrt{\frac{2}{(4 k+1) \pi}}, k \in \mathbb{N}$, we get

$$
\begin{aligned}
\int_{a_{k}}^{b_{k}} f(s) d s & =b_{k}^{2} \sin \left(\frac{1}{b_{k}^{2}}\right)-a_{k}^{2} \sin \left(\frac{1}{a_{k}^{2}}\right) \\
& =\frac{2}{(4 k+1) \pi} \sin \left(\frac{\pi}{2}+2 k \pi\right)-\frac{1}{(2 k+1) \pi} \sin (\pi+2 k \pi)=\frac{2}{(4 k+1) \pi} .
\end{aligned}
$$

Since the intervals $\left[a_{k}, b_{k}\right], k \in \mathbb{N}$, are pairwise disjoint and $\bigcup_{k=1}^{\infty}\left[a_{k}, b_{k}\right] \subset[0,1]$, we have

$$
\int_{0}^{1}|f(s)| d s \geq \sum_{k=1}^{\infty} \int_{a_{k}}^{b_{k}}|f(s)| d s \geq \sum_{k=1}^{\infty}\left|\int_{a_{k}}^{b_{k}} f(s) d s\right|=\frac{2}{\pi} \sum_{k=1}^{\infty} \frac{1}{4 k+1}=+\infty .
$$

Therefore, $f$ is not absolutely integrable on $[0,1]$ and this implies that $f$ is not Lesbegue integrable on $[0,1]$. 
CHAPTER

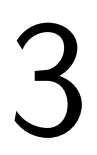

MEASURE FUNCTIONAL INTEGRAL

EQUATIONS

This chapter is dedicated to the study of existence and uniqueness of periodic solutions for measure functional differential equations (measure FDEs) of type:

$$
x(t)=x(0)+\int_{0}^{t} f\left(s, x_{s}\right) d s+\int_{0}^{t} g\left(s, x_{s}\right) d u(s),
$$

defined for every $t \in \mathbb{R}$.

The next definition concerns the concept of a solution of the measure FDE (3.1).

Definition 3.0.1. A function $x: \mathbb{R} \rightarrow \mathbb{R}^{n}$ is said to be a solution of the measure FDE (3.1) if $x \in G^{-}\left(\mathbb{R}, \mathbb{R}^{n}\right)$ and $x$ satisfies (3.1) for every $t \in \mathbb{R}$.

\subsection{Existence of periodic solutions}

In order to study the existence of periodic solutions of the measure FDE (3.1), we shall consider its equivalent form

$$
x(t)=x\left(s_{0}\right)+\int_{s_{0}}^{t} f\left(s, x_{s}\right) d s+\int_{s_{0}}^{t} g\left(s, x_{s}\right) d u(s), \quad t \in \mathbb{R},
$$

where $s_{0} \in[0, T]$ is a point of continuity of $u$ for some $T>0$.

Note that the space $G_{T}^{-}\left(\mathbb{R}, \mathbb{R}^{n}\right)$ is Banach when endowed with the norm

$$
|x|_{T}=\sup _{t \in\left[s_{0}, s_{0}+T\right]}\|x(t)\| .
$$

In what follows, we present the general conditions that we shall consider in this section.

(H1) $u: \mathbb{R} \rightarrow \mathbb{R}$ is a left-continuous regulated function on $\mathbb{R}$, continuous at $s_{0} \in[0, T]$, and there exists $c \in \mathbb{R}$ such that $u(t+T)=u(t)+c$ for all $t \in \mathbb{R}$; 
(H2) $f, g: \mathbb{R} \times G^{-}\left(\mathbb{R}, \mathbb{R}^{n}\right) \rightarrow \mathbb{R}^{n}$ are $T$-periodic functions with respect to the first variable such that, for each $x \in G_{T}^{-}\left(\mathbb{R}, \mathbb{R}^{n}\right)$, the map $t \mapsto f\left(t, x_{t}\right)$ is locally Perron integrable over $\mathbb{R}$ and the map $t \mapsto g\left(t, x_{t}\right)$ is locally Perron-Stieltjes integrable over $\mathbb{R}$ with respect to $u$;

(H3) there exists a function $h \in G^{-}(\mathbb{R}, \mathbb{R})$ continuous at $s_{0}$ such that, for any $\mu>0$, one can find a number $N_{\mu}>0$ satisfying

$$
\left\|\int_{t_{1}}^{t_{2}} f\left(s, x_{s}\right) d s\right\| \leq N_{\mu}\left|h\left(t_{2}\right)-h\left(t_{1}\right)\right|
$$

and

$$
\left\|\int_{t_{1}}^{t_{2}} g\left(s, x_{s}\right) d u(s)\right\| \leq N_{\mu}\left|h\left(t_{2}\right)-h\left(t_{1}\right)\right|
$$

whenever $t_{1}, t_{2} \in\left[s_{0}, s_{0}+T\right]$ and $x \in G_{T}^{-}\left(\mathbb{R}, \mathbb{R}^{n}\right)$ with $|x|_{T} \leq \mu$;

(H4) given $\mu>0$, there exist a non-negative locally Perron integrable function $M_{\mu}: \mathbb{R} \rightarrow \mathbb{R}$ and a non-negative locally Perron-Stieltjes integrable function $L_{\mu}: \mathbb{R} \rightarrow \mathbb{R}$ with respect to $u$ such that, for all $t_{1}, t_{2} \in\left[s_{0}, s_{0}+T\right], t_{1} \leq t_{2}$,

$$
\left\|\int_{t_{1}}^{t_{2}}\left[f\left(s, x_{s}\right)-f\left(s, y_{s}\right)\right] d s\right\| \leq \int_{t_{1}}^{t_{2}} M_{\mu}(s)|x-y|_{T} d s
$$

and

$$
\left\|\int_{t_{1}}^{t_{2}}\left[g\left(s, x_{s}\right)-g\left(s, y_{s}\right)\right] d u(s)\right\| \leq \int_{t_{1}}^{t_{2}} L_{\mu}(s)|x-y|_{T} d u(s),
$$

for all $x, y \in G_{T}^{-}\left(\mathbb{R}, \mathbb{R}^{n}\right)$ such that $|x|_{T} \leq \mu$ and $|y|_{T} \leq \mu$.

Associated with equation (3.2), let us consider the following family of measure functional differential equations

$$
x(t)=\lambda x\left(s_{0}\right)+\int_{s_{0}}^{t} \lambda f\left(s, x_{s}\right) d s+\int_{s_{0}}^{t} \lambda g\left(s, x_{s}\right) d u(s), \quad \lambda \in(0,1]
$$

defined for every $t \in \mathbb{R}$.

The idea is to consider the family of measure FDEs (3.3) and make use of Theorem 2.1.8 to establish sufficient conditions to obtain periodic solutions of the measure FDE (3.2). We start by presenting some auxiliary results.

Lemma 3.1.1. The continuity points of $u$ are continuity points of the solutions of the family of measure FDEs (3.3).

Proof. Let $t_{0} \in \mathbb{R}$ be a point of continuity of $u$ and $x$ be a solution of (3.3) for some $\lambda \in(0,1]$. By Theorem 2.4.5, we have

$$
x\left(t_{0}^{+}\right)=\lambda x\left(s_{0}\right)+\int_{s_{0}}^{t_{0}} \lambda f\left(s, x_{s}\right) d s+\int_{s_{0}}^{t_{0}} \lambda g\left(s, x_{s}\right) d u(s)+\lambda g\left(t_{0}, x_{t_{0}}\right) \Delta^{+} u\left(t_{0}\right)
$$


and

$$
x\left(t_{0}^{-}\right)=\lambda x\left(s_{0}\right)+\int_{s_{0}}^{t_{0}} \lambda f\left(s, x_{s}\right) d s+\int_{s_{0}}^{t_{0}} \lambda g\left(s, x_{s}\right) d u(s)-\lambda g\left(t_{0}, x_{t_{0}}\right) \Delta^{-} u\left(t_{0}\right) .
$$

Now, by (3.3), we get

$$
x\left(t_{0}\right)=\lambda x\left(s_{0}\right)+\int_{s_{0}}^{t_{0}} \lambda f\left(s, x_{s}\right) d s+\int_{s_{0}}^{t_{0}} \lambda g\left(s, x_{s}\right) d u(s) .
$$

Consequently, using (3.4), (3.5) and (3.6), we obtain

$$
x\left(t_{0}^{+}\right)-x\left(t_{0}\right)=\lambda g\left(t_{0}, x_{t_{0}}\right) \Delta^{+} u\left(t_{0}\right)=0
$$

and

$$
x\left(t_{0}^{-}\right)-x\left(t_{0}\right)=-\lambda g\left(t_{0}, x_{t_{0}}\right) \Delta^{-} u\left(t_{0}\right)=0 .
$$

Lemma 3.1.2. Let $K_{\beta}=2 N_{\beta}$ for $\beta>0$ fixed, where $N_{\beta}$ comes from condition (H3). Then

$$
Y_{K_{\beta}}=\left\{x \in G_{T}^{-}\left(\mathbb{R}, \mathbb{R}^{n}\right):\left\|x\left(t_{1}\right)-x\left(t_{2}\right)\right\| \leq K_{\beta}\left|h\left(t_{1}\right)-h\left(t_{2}\right)\right|, t_{1}, t_{2} \in\left(s_{0}, s_{0}+T\right]\right\}
$$

is a nonempty convex closed subset of $G_{T}^{-}\left(\mathbb{R}, \mathbb{R}^{n}\right)$.

Proof. It is not difficult to see that $Y_{K_{\beta}}$ is closed and convex. Let us show that $Y_{K_{\beta}}$ is a nonempty set. Take $x_{0} \in \mathbb{R}^{n}$ with $\left\|x_{0}\right\| \leq K_{\beta}$. Now, define a function $z:\left(s_{0}, s_{0}+T\right] \rightarrow \mathbb{R}^{n}$ by $z(t)=x_{0} h(t)$ and let $x: \mathbb{R} \rightarrow \mathbb{R}^{n}$ be its $T$-periodic extension on $\mathbb{R}$. Thus, for $t_{1}, t_{2} \in\left(s_{0}, s_{0}+T\right]$, we have

$$
\left\|x\left(t_{1}\right)-x\left(t_{2}\right)\right\|=\left\|z\left(t_{1}\right)-z\left(t_{2}\right)\right\|=\left\|x_{0}\right\|\left|h\left(t_{1}\right)-h\left(t_{2}\right)\right| \leq K_{\beta}\left|h\left(t_{1}\right)-h\left(t_{2}\right)\right| .
$$

Hence, $x \in Y_{K_{\beta}}$ and the proof is complete.

According to Lemma 3.1.1, we need to find $T$-periodic solutions of (3.2) which are continuous at $s_{0}$. In this way, let us consider the space $G_{T}^{0-}\left(\mathbb{R}, \mathbb{R}^{n}\right)$ that represents the set of all functions in $G_{T}^{-}\left(\mathbb{R}, \mathbb{R}^{n}\right)$ which are continuous at $s_{0}$. Note that $G_{T}^{0-}\left(\mathbb{R}, \mathbb{R}^{n}\right)$ is a Banach space with the norm $|\cdot|_{T}$.

Now, define the set

$$
X_{\beta}=\left\{x \in Y_{K_{\beta}} \cap G_{T}^{0-}\left(\mathbb{R}, \mathbb{R}^{n}\right):|x|_{T} \leq \beta\right\},
$$

where the set $Y_{K_{\beta}}$ comes from Lemma 3.1.2. Also, we define the map $H:[0,1] \times X_{\beta} \rightarrow Y_{K_{\beta}}$ by

$$
H(\lambda, x)(t)=\lambda x\left(s_{0}\right)+\int_{s_{0}+k T}^{t} \lambda f\left(s, x_{s}\right) d s+\int_{s_{0}+k T}^{t} \lambda g\left(s, x_{s}\right) d u(s),
$$

for all $t \in\left(s_{0}+k T, s_{0}+(k+1) T\right], k \in \mathbb{Z}$.

Claim: $H$ is well-defined. 
In fact, let $x \in X_{\beta}$ and $\lambda \in[0,1]$. By conditions (H1)-(H2) and Theorems 2.4.13 and 2.4.5, the map $H(\lambda, x) \in G^{-}\left(\mathbb{R}, \mathbb{R}^{n}\right)$.

Since $f, g: \mathbb{R} \times G^{-}\left(\mathbb{R}, \mathbb{R}^{n}\right) \rightarrow \mathbb{R}^{n}$ are $T$-periodic functions with respect to the first variable (see condition (H2)) and $u(t+T)=u(t)+c$ for all $t \in \mathbb{R}$ (see condition (H1)), we may use Theorem 2.4.9 to obtain

$$
\begin{aligned}
& \int_{s_{0}+(k+1) T}^{t+T} f\left(s, x_{s}\right) d s+\int_{s_{0}+(k+1) T}^{t+T} g\left(s, x_{s}\right) d u(s)= \\
& =\int_{s_{0}+k T}^{t} f\left(s+T, x_{s+T}\right) d s+\int_{s_{0}+k T}^{t} g\left(s+T, x_{s+T}\right) d u(s+T) \\
& =\int_{s_{0}+k T}^{t} f\left(s+T, x_{s+T}\right) d s+\int_{s_{0}+k T}^{t} g\left(s+T, x_{s+T}\right) d(u(s)+c) \\
& =\int_{s_{0}+k T}^{t} f\left(s, x_{s}\right) d s+\int_{s_{0}+k T}^{t} g\left(s, x_{s}\right) d u(s),
\end{aligned}
$$

for all $t \in \mathbb{R}$ and $k \in \mathbb{Z}$, which implies that $H(\lambda, x)(t)=H(\lambda, x)(t+T)$ for all $t \in \mathbb{R}$.

Lastly, given $t_{1}, t_{2} \in\left(s_{0}, s_{0}+T\right]$, it follows by condition $(\mathrm{H} 3)$ and by the fact that $K_{\beta}=$ $2 N_{\beta}$, that

$$
\begin{aligned}
& \left\|H(\lambda, x)\left(t_{2}\right)-H(\lambda, x)\left(t_{1}\right)\right\| \leq \\
& \leq\left\|\int_{t_{1}}^{t_{2}} \lambda f\left(s, x_{s}\right) d s\right\|+\left\|\int_{t_{1}}^{t_{2}} \lambda g\left(s, x_{s}\right) d u(s)\right\| \\
& \leq 2 N_{\beta}\left|h\left(t_{2}\right)-h\left(t_{1}\right)\right| \\
& =K_{\beta}\left|h\left(t_{2}\right)-h\left(t_{1}\right)\right| .
\end{aligned}
$$

Therefore, $H(\lambda, x) \in Y_{K_{\beta}}$ for all $x \in X_{\beta}$ and $\lambda \in[0,1]$.

Remark 3.1.3. For each $x \in X_{\beta}$ and $\lambda \in[0,1]$, it follows from the previous inequality that $H(\lambda, x): \mathbb{R} \rightarrow \mathbb{R}^{n}$ is continuous at every point of $\mathbb{R}$ for which $h$ is continuous.

As shown in the next lemma, for each $x \in X_{\beta}$, the map $H(\cdot, x):[0,1] \rightarrow Y_{K_{\beta}}$ is continuous.

Lemma 3.1.4. For each $x \in X_{\beta}$, the map $H(\cdot, x):[0,1] \rightarrow Y_{K_{\beta}}$ is continuous at every $\lambda \in[0,1]$.

Proof. Let $x \in X_{\beta}, t \in\left(s_{0}, s_{0}+T\right]$, and $\lambda_{1}, \lambda_{2} \in[0,1]$. By (3.7), we have

$$
\begin{aligned}
& \left\|H\left(\lambda_{2}, x\right)(t)-H\left(\lambda_{1}, x\right)(t)\right\|= \\
& =\left|\lambda_{2}-\lambda_{1}\right|\left\|x\left(s_{0}\right)+\int_{s_{0}}^{t} f\left(s, x_{s}\right) d s+\int_{s_{0}}^{t} g\left(s, x_{s}\right) d u(s)\right\| \\
& =\left|\lambda_{2}-\lambda_{1}\right|\|H(1, x)(t)\| .
\end{aligned}
$$

Since $H(\lambda, x)\left(s_{0}\right)=H(\lambda, x)\left(s_{0}+T\right)$, for all $x \in X_{\beta}$ and $\lambda \in[0,1]$, applying the supremum over $\left[s_{0}, s_{0}+T\right]$, we obtain

$$
\left|H\left(\lambda_{2}, x\right)-H\left(\lambda_{1}, x\right)\right|_{T} \leq\left|\lambda_{2}-\lambda_{1}\right||H(1, x)|_{T}
$$

which concludes the proof. 
Lemma 3.1.5 concerns the continuity of the map $X_{\beta} \ni x \mapsto H(\lambda, x)$.

Lemma 3.1.5. For each $\lambda \in[0,1]$, the map $H(\lambda, \cdot): X_{\beta} \rightarrow Y_{K_{\beta}}$ given in (3.7) is uniformly continuous on $X_{\beta}$.

Proof. Let $\lambda \in[0,1], t \in\left(s_{0}, s_{0}+T\right]$, and $x, y \in X_{\beta}$. Using condition (H4), we obtain

$$
\begin{aligned}
& \|H(\lambda, x)(t)-H(\lambda, y)(t)\| \leq \\
& \leq\left\|x\left(s_{0}\right)-y\left(s_{0}\right)\right\|+\left\|\int_{s_{0}}^{t}\left[f\left(s, x_{s}\right)-f\left(s, y_{s}\right)\right] d s\right\|+\left\|\int_{s_{0}}^{t}\left[g\left(s, x_{s}\right)-g\left(s, y_{s}\right)\right] d u(s)\right\| \\
& \leq|x-y|_{T}+\int_{s_{0}}^{t} M_{\beta}(s)|x-y|_{T} d s+\int_{s_{0}}^{t} L_{\beta}(s)|x-y|_{T} d u(s) \\
& \leq\left(1+\int_{s_{0}}^{t} M_{\beta}(s) d s+\int_{s_{0}}^{t} L_{\beta}(s) d u(s)\right)|x-y|_{T} .
\end{aligned}
$$

Since $H(\lambda, x)\left(s_{0}\right)=H(\lambda, x)\left(s_{0}+T\right)$ and $H(\lambda, y)\left(s_{0}\right)=H(\lambda, y)\left(s_{0}+T\right)$, we conclude that

$$
|H(\lambda, x)-H(\lambda, y)|_{T} \leq\left(1+\int_{s_{0}}^{s_{0}+T} M_{\beta}(s) d s+\int_{s_{0}}^{s_{0}+T} L_{\beta}(s) d u(s)\right)|x-y|_{T},
$$

which implies that $H(\lambda, \cdot)$ is uniformly continuous on $X_{\beta}$ for each $\lambda \in[0,1]$.

Lemma 3.1.6. Let $\lambda \in[0,1]$ and $x \in X_{\beta}$. Then,

$$
\lim _{t \rightarrow s_{0}^{+}} H(\lambda, x)(t)=\lambda x\left(s_{0}\right)
$$

Proof. Let $t \in\left(s_{0}, s_{0}+T\right]$ and $x \in X_{\beta}$. According to (3.7), we may write

$$
H(\lambda, x)(t)=\lambda x\left(s_{0}\right)+\int_{s_{0}}^{t} \lambda f\left(s, x_{s}\right) d s+\int_{s_{0}}^{t} \lambda g\left(s, x_{s}\right) d u(s) .
$$

By Theorem 2.4.13, we have $\lim _{t \rightarrow s_{0}^{+}} \int_{s_{0}}^{t} f\left(s, x_{s}\right) d s=0$. Using Theorem 2.4.5, we conclude that

$$
\lim _{t \rightarrow s_{0}^{+}} \int_{s_{0}}^{t} g\left(s, x_{s}\right) d u(s)=\int_{s_{0}}^{t_{0}} g\left(s, x_{s}\right) d u(s)+g\left(s_{0}, x_{s_{0}}\right) \Delta^{+} u\left(s_{0}\right)=g\left(s_{0}, x_{s_{0}}\right) \Delta^{+} u\left(s_{0}\right)=0,
$$

as $u$ is continuous at $s_{0}$. Therefore, the result is proved.

Lemma 3.1.7. Let $\lambda \in(0,1]$. If $x \in X_{\beta}$ is a fixed point of $H(\lambda, \cdot)$, then

$$
\int_{t}^{t+T} f\left(s, x_{s}\right) d s+\int_{t}^{t+T} g\left(s, x_{s}\right) d u(s)=0,
$$

for all $t \in \mathbb{R}$.

Proof. Fix $\lambda \in(0,1]$ and assume that $x \in X_{\beta}$ is a fixed point of $H(\lambda, \cdot)$. For $t \in\left(s_{0}, s_{0}+T\right]$, we may write

$$
x(t)=H(\lambda, x)(t)=\lambda x\left(s_{0}\right)+\int_{s_{0}}^{t} \lambda f\left(s, x_{s}\right) d s+\int_{s_{0}}^{t} \lambda g\left(s, x_{s}\right) d u(s) .
$$


Using the periodicity of $x$ and (3.8), we obtain

$$
x\left(s_{0}\right)=x\left(s_{0}+T\right)=\lambda x\left(s_{0}\right)+\int_{s_{0}}^{s_{0}+T} \lambda f\left(s, x_{s}\right) d s+\int_{s_{0}}^{s_{0}+T} \lambda g\left(s, x_{s}\right) d u(s) .
$$

By Lemma 3.1.6 and equation (3.8), we get

$$
x\left(s_{0}^{+}\right)=\lambda x\left(s_{0}\right)
$$

Consequently, using (3.9) and (3.10), we have

$$
\int_{s_{0}}^{s_{0}+T} \lambda f\left(s, x_{s}\right) d s+\int_{s_{0}}^{s_{0}+T} \lambda g\left(s, x_{s}\right) d u(s)=x\left(s_{0}\right)-\lambda x\left(s_{0}\right)=-\Delta^{+} x\left(s_{0}\right)=0,
$$

since $x$ is continuous at $s_{0}\left(x \in X_{\beta}\right)$.

Now, let $t \in \mathbb{R}$. Using the periodicity of $f$ and $x$, we obtain

$$
\begin{aligned}
\int_{t}^{t+T} f\left(s, x_{s}\right) d s & =\int_{t}^{s_{0}+k T} f\left(s, x_{s}\right) d s+\int_{s_{0}+k T}^{s_{0}+(k+1) T} f\left(s, x_{s}\right) d s+\int_{s_{0}+(k+1) T}^{t+T} f\left(s, x_{s}\right) d s \\
& =\int_{t}^{s_{0}+k T} f\left(s, x_{s}\right) d s+\int_{s_{0}}^{s_{0}+T} f\left(s, x_{s}\right) d s+\int_{s_{0}+k T}^{t} f\left(s, x_{s}\right) d s \\
& =\int_{s_{0}}^{s_{0}+T} f\left(s, x_{s}\right) d s,
\end{aligned}
$$

and, analogously,

$$
\int_{t}^{t+T} g\left(s, x_{s}\right) d u(s)=\int_{s_{0}}^{s_{0}+T} g\left(s, x_{s}\right) d u(s),
$$

as $g$ is periodic with respect to the first variable and $u(t+T)=u(t)+c$ for all $t \in \mathbb{R}$. Hence, the result follows from (3.11) and the previous equalities.

In Lemma 3.1.8, we prove the compactness of the map $H$.

Lemma 3.1.8. The map $H:[0,1] \times X_{\beta} \rightarrow Y_{K_{\beta}}$, defined in (3.7), is compact.

Proof. It is enough to prove that $X_{\beta}$ is compact. In order to do that, let $\left\{u_{j}\right\}_{j \in \mathbb{N}} \subset X_{\beta}$ be a sequence. By the periodicity of the functions of $X_{\beta}$, we need to show that $\left\{\left.u_{j}\right|_{\left[s_{0}, s_{0}+T\right]}\right\}_{j \in \mathbb{N}}$ admits a uniformly convergent subsequence defined on $\left[s_{0}, s_{0}+T\right]$. Since $\left\{u_{j}\right\}_{j \in \mathbb{N}} \subset X_{\beta}$, it follows that $\left\{u_{j}\right\}_{j \in \mathbb{N}}$ is uniformly bounded and

$$
\left\|u_{j}\left(t_{1}\right)-u_{j}\left(t_{2}\right)\right\| \leq K_{\beta}\left|h\left(t_{1}\right)-h\left(t_{2}\right)\right|
$$

for all $t_{1}, t_{2} \in\left(s_{0}, s_{0}+T\right]$ and for all $j \in \mathbb{N}$. Since $h \in G^{-}(\mathbb{R}, \mathbb{R})$ due to condition (H3), we have $\left\{u_{j}\right\}_{j \in \mathbb{N}}$ is equiregulated on $\left[s_{0}, s_{0}+T\right]$.

By Theorem 2.3.7, $\left\{\left.u_{j}\right|_{\left[s_{0}, s_{0}+T\right]}\right\}_{j \in \mathbb{N}}$ admits a uniformly convergent subsequence on $\left[s_{0}, s_{0}+T\right]$. Let $u_{0} \in G^{0-}\left(\left[s_{0}, s_{0}+T\right], \mathbb{R}^{n}\right)$ be its limit. Since $u_{0}\left(s_{0}\right)=u_{0}\left(s_{0}+T\right)$, the function $\bar{u}(t)=u_{0}(t-k T), s_{0}+k T<t \leq s_{0}+(k+1) T, k \in \mathbb{Z}$, is the limit of a subsequence of $\left\{u_{j}\right\}_{j \in \mathbb{N}}$, which completes the proof. 
Next, we exhibit the main result of this section. By using Theorem 2.1.8, we obtain a criterion to guarantee the existence of at least one $T$-periodic solution for the measure FDE (3.1). In order to use Theorem 2.1.8, we will consider $E=G_{T}^{-}\left(\mathbb{R}, \mathbb{R}^{n}\right), X=X_{\beta}$, and $Y=Y_{K_{\beta}}$. Recall from Lemma 3.1.2 that $Y_{K_{\beta}}$ is a nonempty convex closed subset of $G_{T}^{-}\left(\mathbb{R}, \mathbb{R}^{n}\right)$. Moreover, it is not difficult to check that $X_{\beta}$ is a closed subset of $Y_{K_{\beta}}$.

Theorem 3.1.9. Assume that conditions $(\mathrm{H} 1)-(\mathrm{H} 4)$ hold. If there exists a constant $\beta>0$ such that $\|x(t)\|<\beta$ for all $t \in \mathbb{R}$ whenever $x(t)$ is a $T$-periodic solution of (3.3), with $\lambda \in(0,1]$, then the measure FDE (3.2) has at least one $T$-periodic solution.

Proof. By Lemma 3.1.8, the map $H:[0,1] \times X_{\beta} \rightarrow Y_{K_{\beta}}$ defined in (3.7) is a $T$-periodic completely continuous homotopy. Note that $H(0, \cdot)=0$ and if $x_{\lambda}^{*} \in X_{\beta}$ is a fixed point of $H(\lambda, \cdot)$, then for all $t \in\left(s_{0}+k T, s_{0}+(k+1) T\right], k \in \mathbb{Z}$,

$$
x_{\lambda}^{*}(t)=\lambda x_{\lambda}^{*}\left(s_{0}\right)+\int_{s_{0}+k T}^{t} \lambda f\left(s,\left(x_{\lambda}^{*}\right)_{s}\right) d s+\int_{s_{0}+k T}^{t} \lambda g\left(s,\left(x_{\lambda}^{*}\right)_{s}\right) d u(s) .
$$

On the other hand, assuming without loss of generality that $k \geq 0$, it follows by Lemma 3.1.7 that

$$
\begin{aligned}
& \int_{s_{0}}^{t} f\left(s,\left(x_{\lambda}^{*}\right)_{s}\right) d s+\int_{s_{0}}^{t} g\left(s,\left(x_{\lambda}^{*}\right)_{s}\right) d u(s)= \\
& =\sum_{j=0}^{k-1} \int_{s_{0}+j T}^{s_{0}+(j+1) T} f\left(s,\left(x_{\lambda}^{*}\right)_{s}\right) d s+\int_{s_{0}+k T}^{t} f\left(s,\left(x_{\lambda}^{*}\right)_{s}\right) d s \\
& \quad+\sum_{j=0}^{k-1} \int_{s_{0}+j T}^{s_{0}+(j+1) T} g\left(s,\left(x_{\lambda}^{*}\right)_{s}\right) d u(s)+\int_{s_{0}+k T}^{t} g\left(s,\left(x_{\lambda}^{*}\right)_{s}\right) d u(s) \\
& =\int_{s_{0}+k T}^{t} f\left(s,\left(x_{\lambda}^{*}\right)_{s}\right) d s+\int_{s_{0}+k T}^{t} g\left(s,\left(x_{\lambda}^{*}\right)_{s}\right) d u(s) .
\end{aligned}
$$

Consequently,

$$
x_{\lambda}^{*}(t)=\lambda x_{\lambda}^{*}\left(s_{0}\right)+\int_{s_{0}}^{t} \lambda f\left(s,\left(x_{\lambda}^{*}\right)_{s}\right) d s+\int_{s_{0}}^{t} \lambda g\left(s,\left(x_{\lambda}^{*}\right)_{s}\right) d u(s),
$$

that is, $x_{\lambda}^{*} \in X_{\beta}$ is a $T$-periodic solution of (3.3). Thus, by hypothesis, $H(\lambda, \cdot)$ has no fixed points in $\partial X_{\beta}=\left\{\phi \in Y_{K_{\beta}}:\|\phi\|_{T}=\beta\right\}$ for all $\lambda \in(0,1]$. By Theorem 2.1.8-(i), $H(1, \cdot)$ has a fixed point in $X_{\beta} \backslash \partial X_{\beta}$. Therefore, the measure FDE (3.2) admits a $T$-periodic solution.

Theorem 3.1.10 deals with sufficient conditions for the uniqueness of $T$-periodic solutions of the measure FDE (3.2).

Theorem 3.1.10. Assume that condition (H4) holds. If $x, y \in G_{T}^{-}\left(\mathbb{R}, \mathbb{R}^{n}\right)$ are $T$-periodic solutions of the measure FDE (3.2) such that $x\left(s_{0}\right)=y\left(s_{0}\right)$ and, in addition, there is a constant $\beta>0$ such that $|x|_{T} \leq \beta,|y|_{T} \leq \beta$, and

$$
\int_{s_{0}}^{s_{0}+T} M_{\beta}(s) d s+\int_{s_{0}}^{s_{0}+T} L_{\beta}(s) d u(s)<1,
$$

then $x(t)=y(t)$ for all $t \in \mathbb{R}$. 
Proof. Let $t \in\left[s_{0}, s_{0}+T\right]$. Using condition (H4), we have

$$
\begin{aligned}
\|x(t)-y(t)\| & \leq\left\|\int_{s_{0}}^{t}\left[f\left(s, x_{s}\right)-f\left(s, y_{s}\right)\right] d s\right\|+\left\|\int_{s_{0}}^{t}\left[g\left(s, x_{s}\right)-g\left(s, y_{s}\right)\right] d u(s)\right\| \\
& \leq\left(\int_{s_{0}}^{t} M_{\beta}(s) d s+\int_{s_{0}}^{t} L_{\beta}(s) d u(s)\right)|x-y|_{T} .
\end{aligned}
$$

Consequently, by applying the supremum on both side of the previous inequality on $\left[s_{0}, s_{0}+T\right]$, we obtain

$$
\left(1-\int_{s_{0}}^{s_{0}+T} M_{\beta}(s) d s-\int_{s_{0}}^{s_{0}+T} L_{\beta}(s) d u(s)\right)|x-y|_{T} \leq 0 .
$$

Since $1-\int_{s_{0}}^{s_{0}+T} M_{\beta}(s) d s-\int_{s_{0}}^{s_{0}+T} L_{\beta}(s) d u(s)>0$, we get $|x-y|_{T}=0$, that is, $x(t)=y(t)$ for all $t \in\left[s_{0}, s_{0}+T\right]$. By the periodicity, we can infer that $x(t)=y(t)$ for all $t \in \mathbb{R}$.

\subsection{Priori bounds of the periodic solution}

As stated in Theorem 3.1.9, the $T$-periodic solutions of (3.3) need to be bounded by the same constant. In this section, we present some results that give sufficient conditions to obtain a limiting factor for all possible $T$-periodic solutions of the auxiliary measure FDEs (3.3), with $\lambda \in(0,1]$.

Remark 3.2.1. Since $u$ is continuous at $s_{0}$, there exists $\delta_{u} \in(0, T)$ such that $u$ is continuous on $\left[s_{0}-\delta_{u}, s_{0}+\delta_{u}\right]$. In the next results, we denote by $\Lambda_{u}$ the set of continuity points of $u$ on $\mathbb{R}$.

Proposition 3.2.2. Under the hypotheses $(\mathrm{H} 1)-(\mathrm{H} 4)$, assume that the following conditions hold:

(i) there exists $N>0$ such that

$$
\left\|\int_{t_{1}}^{t_{2}} g\left(s, x_{s}\right) d u(s)\right\| \leq N \quad \text { and } \quad\left\|\int_{t_{1}}^{t_{2}} f\left(s, x_{s}\right) d s\right\| \leq N
$$

for all $t_{1}, t_{2} \in\left[s_{0}, s_{0}+T\right]$ and $x \in G_{T}^{-}\left(\mathbb{R}, \mathbb{R}^{n}\right) ;$

(ii) there are $j \in\{1,2, \ldots, n\}, \gamma>0, r \in \mathbb{R}$ and $M>0$ such that $\|x(t-r)\| \leq M\left|f_{j}\left(t, x_{t}\right)\right|^{\gamma}$ for all $t \in\left[s_{0}, s_{0}+T\right]$ and $x \in G_{T}^{-}\left(\mathbb{R}, \mathbb{R}^{n}\right)$, where $f_{j}$ denotes the $j$ th coordinate of $f$;

(iii) there exist $\delta \in\left(0, \delta_{u}\right]$ and $s_{1} \in\left[s_{0}, s_{0}+T\right)$, with $s_{1}+\delta<s_{0}+T$, such that the map $t \mapsto f\left(t, x_{t}\right)$ is continuous on $\left[s_{1}, s_{1}+\delta\right]$ whenever $x: \mathbb{R} \rightarrow \mathbb{R}^{n}$ is continuous on $\Lambda_{u}$, where $\delta_{u}$ and $\Lambda_{u}$ come from Remark 3.2.1.

Then, there exists a constant $\beta>0$ such that $\|x(t)\|<\beta$ for all $t \in \mathbb{R}$, whenever $x(t)$ is a $T$-periodic solution of the auxiliary measure FDE (3.3), with $\lambda \in(0,1]$.

Proof. Let $x_{\lambda}(t)$ be an arbitrary $T$-periodic solution of (3.3) with $0<\lambda \leq 1$. By Remark 3.1.1, $x_{\lambda}(t)$ is also continuous on $\Lambda_{u}$. Now, using condition (iii), the map $t \mapsto f\left(t,\left(x_{\lambda}\right)_{t}\right)$ is continuous 
on the interval $\left[s_{1}, s_{1}+\delta\right]$. By the Mean Value Theorem for integrals (see Theorem 2.4.23), there exists $\xi \in\left(s_{1}, s_{1}+\delta\right)$ such that

$$
f_{j}\left(\xi,\left(x_{\lambda}\right) \xi\right)=\frac{1}{\delta} \int_{s_{1}}^{s_{1}+\delta} f_{j}\left(s,\left(x_{\lambda}\right)_{s}\right) d s,
$$

where $j$ comes from condition (ii). Consider the norm $\|z\|_{1}=\sum_{j=1}^{n}\left|z_{j}\right|, z \in \mathbb{R}^{n}$, and take $K>0$ such that $\|\cdot\|_{1} \leq K\|\cdot\|$. Let $k_{0} \in \mathbb{Z}$ be such that $\xi_{0}:=\xi-r-k_{0} T \in\left[s_{0}, s_{0}+T\right]$. Using the periodicity of $x_{\lambda}$, conditions (i)-(ii) and (3.12), we obtain

$$
\begin{aligned}
\left\|x_{\lambda}\left(\xi_{0}\right)\right\|=\left\|x_{\lambda}(\xi-r)\right\| \leq \frac{M}{\delta^{\gamma}}\left|\int_{s_{1}}^{s_{1}+\delta} f_{j}\left(s,\left(x_{\lambda}\right)_{s}\right) d s\right|^{\gamma} \\
\leq \frac{K^{\gamma} M}{\delta^{\gamma}}\left\|\int_{s_{1}}^{s_{1}+\delta} f\left(s,\left(x_{\lambda}\right)_{s}\right) d s\right\|^{\gamma} \leq \frac{K^{\gamma} M N^{\gamma}}{\delta^{\gamma}} .
\end{aligned}
$$

On the other hand, since

$$
x_{\lambda}(t)=\lambda x_{\lambda}\left(\xi_{0}\right)+\int_{\xi_{0}}^{t} \lambda f\left(s,\left(x_{\lambda}\right)_{s}\right) d s+\int_{\xi_{0}}^{t} \lambda g\left(s,\left(x_{\lambda}\right)_{s}\right) d u(s)
$$

and conditions (i) and (3.13) hold, we conclude that

$$
\begin{aligned}
\left\|x_{\lambda}(t)\right\| & \leq\left\|x_{\lambda}\left(\xi_{0}\right)\right\|+\left\|\int_{\xi_{0}}^{t} f\left(s,\left(x_{\lambda}\right)_{s}\right) d s\right\|+\left\|\int_{\xi_{0}}^{t} g\left(s,\left(x_{\lambda}\right)_{s}\right) d u(s)\right\| \\
& \leq \frac{K^{\gamma} M N^{\gamma}}{\delta^{\gamma}}+2 N
\end{aligned}
$$

for all $t \in\left[s_{0}, s_{0}+T\right]$. Taking $\beta=\frac{K^{\gamma} M N^{\gamma}}{\delta^{\gamma}}+2 N$, we have the desired result.

Remark 3.2.3. If condition (ii) of Proposition 3.2 .2 is replaced by condition:

- there are $\gamma>0, r \in \mathbb{R}$ and $M>0$ such that $\left|x_{j}(t-r)\right| \leq M\left|f_{j}\left(t, x_{t}\right)\right|^{\gamma}$ for all $j \in\{1, \ldots, n\}$, $t \in\left[s_{0}, s_{0}+T\right]$ and all $x \in G_{T}^{-}\left(\mathbb{R}, \mathbb{R}^{n}\right)$, where $x_{j}\left(f_{j}\right)$ denotes the $j$ th coordinate of $x(f)$,

then Proposition 3.2.2 still holds. In fact, by using the $j$ th coordinate $x_{\lambda}^{j}$ of the solution $x_{\lambda}$ and the notations of the proof of Proposition 3.2.2, we can obtain in (3.13) the following estimate

$$
\left|x_{\lambda}^{j}\left(\xi_{0}\right)\right| \leq \frac{K^{\gamma} M N^{\gamma}}{\delta^{\gamma}}, \quad j=1, \ldots, n
$$

Considering the norm $\|z\|_{2}=\max _{1 \leq j \leq n}\left|z_{j}\right|, z \in \mathbb{R}^{n}$, there is $\tilde{K}>0$ such that $\|\cdot\| \leq \tilde{K}\|\cdot\|_{2}$. Then

$$
\left\|x_{\lambda}\left(\xi_{0}\right)\right\| \leq \tilde{K}\left\|x_{\lambda}\left(\xi_{0}\right)\right\|_{2} \leq \frac{\tilde{K} K^{\gamma} M N^{\gamma}}{\delta^{\gamma}}
$$

and we may set $\beta=\frac{\tilde{K} K^{\gamma} M N^{\gamma}}{\delta^{\gamma}}+2 N$ to conclude the proof.

Proposition 3.2.4. Under the hypotheses (H1)-(H4), assume that conditions (ii) and (iii) of Proposition 3.2.2 holds. Moreover, assume that the following conditions hold: 
(i') there exists $N>0$ such that $\left\|\int_{t_{1}}^{t_{2}} g\left(s, x_{s}\right) d u(s)\right\| \leq N$ for all $t_{1}, t_{2} \in\left[s_{0}, s_{0}+T\right]$ and $x \in$ $G_{T}^{-}\left(\mathbb{R}, \mathbb{R}^{n}\right)$

(ii') $\left\|\int_{t_{1}}^{t_{2}} f\left(s, x_{s}\right) d s\right\| \leq\left\|\int_{s_{0}}^{s_{0}+T} f\left(s, x_{s}\right) d s\right\|$ for all $t_{1}, t_{2} \in\left[s_{0}, s_{0}+T\right]$ and $x \in G_{T}^{-}\left(\mathbb{R}, \mathbb{R}^{n}\right)$.

Then, there exists a constant $\beta>0$ such that $\|x(t)\|<\beta$ for all $t \in \mathbb{R}$, whenever $x(t)$ is a $T$-periodic solution of the auxiliary measure $\operatorname{FDE}$ (3.3), with $\lambda \in(0,1]$.

Proof. By the proof of Proposition 3.2.2, it is enough to show that

$$
\left\|\int_{t_{1}}^{t_{2}} f\left(s,\left(x_{\lambda}\right)_{s}\right) d s\right\| \leq N
$$

for all $t_{1}, t_{2} \in\left[s_{0}, s_{0}+T\right]$, whenever $x_{\lambda}(t)$ is a $T$-periodic solution of (3.3), $0<\lambda \leq 1$. In fact, let $x_{\lambda}(t)$ be an arbitrary $T$-periodic solution of (3.3) with $0<\lambda \leq 1$. By (3.3), we have $x_{\lambda}\left(s_{0}\right)=\lambda x_{\lambda}\left(s_{0}\right)$. Consequently,

$$
\begin{aligned}
& \int_{s_{0}}^{s_{0}+T} f\left(s,\left(x_{\lambda}\right)_{s}\right) d s+\int_{s_{0}}^{s_{0}+T} g\left(s,\left(x_{\lambda}\right)_{s}\right) d u(s) \\
& =x_{\lambda}\left(s_{0}+T\right)-\lambda x_{\lambda}\left(s_{0}\right)=x_{\lambda}\left(s_{0}\right)-\lambda x_{\lambda}\left(s_{0}\right)=0 .
\end{aligned}
$$

Hence, for all $t_{1}, t_{2} \in\left[s_{0}, s_{0}+T\right]$, we have

$$
\left\|\int_{t_{1}}^{t_{2}} f\left(s,\left(x_{\lambda}\right)_{s}\right) d s\right\| \leq\left\|\int_{s_{0}}^{s_{0}+T} f\left(s,\left(x_{\lambda}\right)_{s}\right) d s\right\|=\left\|\int_{s_{0}}^{s_{0}+T} g\left(s,\left(x_{\lambda}\right)_{s}\right) d u(s)\right\| \leq N .
$$

Therefore, follows by Proposition (3.2.2) the desired.

Proposition 3.2.5. Under the hypotheses (H1)-(H4), assume that condition (i') of Proposition 3.2.4 holds. Let $F_{1}, F_{2}: \mathbb{R} \times G^{-}\left(\mathbb{R}, \mathbb{R}^{n}\right) \rightarrow \mathbb{R}^{n}$ be functions such that $f(t, \phi)=F_{1}(t, \phi)+F_{2}(t, \phi)$ for all $(t, \phi) \in \mathbb{R} \times G^{-}\left(\mathbb{R}, \mathbb{R}^{n}\right)$. Further, assume that the following conditions hold:

(iv) there exist $\delta \in\left(0, \delta_{u}\right]$ and $s_{1} \in\left[s_{0}, s_{0}+T\right)$, with $s_{1}+\delta<s_{0}+T$, such that the map $t \mapsto F_{1}\left(t, x_{t}\right)$ is continuous on $\left[s_{1}, s_{1}+\delta\right]$, whenever $x$ is continuous on $\Lambda_{u}$. There are $M>0, r \in \mathbb{R}$ and $j \in\{1,2, \ldots, n\}, n \in \mathbb{N}$, such that

$$
\|x(t-r)\| \leq M F_{1 j}\left(t, x_{t}\right)
$$

for all $t \in\left(s_{0}, s_{0}+T\right)$ and $x \in G_{T}^{-}\left(\mathbb{R}, \mathbb{R}^{n}\right)$, where $F_{1 j}$ is the $j$ th coordinate of the function $F_{1}$;

(v) there exist $0 \leq \eta<1$ and $0 \leq \zeta<1$ such that $\frac{K M \zeta}{\delta}+\eta<1$,

$$
\left\|\int_{t_{1}}^{t_{2}} f\left(s, x_{s}\right) d s\right\| \leq \eta|x|_{T} \quad \text { and } \quad\left\|\int_{t_{1}}^{t_{2}} F_{2}\left(s, x_{s}\right) d s\right\| \leq \zeta|x|_{T},
$$

for all $t_{1}, t_{2} \in\left[s_{0}, s_{0}+T\right]$ and $x \in G_{T}^{-}\left(\mathbb{R}, \mathbb{R}^{n}\right)$. The constant $K>0$ comes from the equivalence $\|\cdot\|_{1} \leq K\|\cdot\|$, where $\|z\|_{1}=\sum_{j=1}^{n}\left|z_{j}\right|, z \in \mathbb{R}^{n}$. 
Then, there exists a constant $\beta>0$ such that $\|x(t)\|<\beta$ for all $t \in \mathbb{R}$, whenever $x(t)$ is a $T$-periodic solution of (3.3), with $\lambda \in(0,1]$.

Proof. Let $x_{\lambda}(t)$ be an arbitrary $T$-periodic solution of (3.3) with $0<\lambda \leq 1$. Note that the equality (3.14) holds. Thus,

$$
\left\|\int_{s_{0}}^{s_{0}+T} f\left(s,\left(x_{\lambda}\right)_{s}\right) d s\right\|=\left\|\int_{s_{0}}^{s_{0}+T} g\left(s,\left(x_{\lambda}\right)_{s}\right) d u(s)\right\| \leq N .
$$

By condition (v) and (3.15), we have

$$
\begin{gathered}
\left\|\int_{s_{0}}^{s_{0}+T} F_{1}\left(s,\left(x_{\lambda}\right)_{s}\right) d s\right\| \leq\left\|\int_{s_{0}}^{s_{0}+T} f\left(s,\left(x_{\lambda}\right)_{s}\right) d s\right\| \\
\quad+\left\|\int_{s_{0}}^{s_{0}+T} F_{2}\left(s,\left(x_{\lambda}\right)_{s}\right) d s\right\| \leq N+\zeta\left|x_{\lambda}\right|_{T} .
\end{gathered}
$$

By Remark 3.2.1, $u$ is continuous on $\left[s_{0}, s_{0}+\delta_{u}\right]$. Using (iv), Lemma 3.1.1 and looking at to the proof of Proposition 3.2.2, one can find $\xi \in\left(s_{1}, s_{1}+\delta\right)$ via the Mean Value Theorem for integrals such that

$$
F_{1 j}\left(\xi,\left(x_{\lambda}\right) \xi\right)=\frac{1}{\delta} \int_{s_{1}}^{s_{1}+\delta} F_{1 j}\left(s,\left(x_{\lambda}\right)_{s}\right) d s .
$$

Let $k_{0} \in \mathbb{Z}$ be such that $\xi_{0}:=\xi-r-k_{0} T \in\left[s_{0}, s_{0}+T\right]$. According to conditions (iv)-(v) and relation (3.16), we obtain

$$
\begin{aligned}
\left\|x_{\lambda}\left(\xi_{0}\right)\right\| & =\left\|x_{\lambda}\left(\xi-r-k_{0} T\right)\right\|=\left\|x_{\lambda}(\xi-r)\right\| \\
& \leq \frac{M}{\delta}\left(\int_{s_{1}}^{s_{1}+\delta} F_{1 j}\left(s,\left(x_{\lambda}\right)_{s}\right) d s\right) \leq \frac{M}{\delta}\left|\int_{s_{0}}^{s_{0}+T} F_{1 j}\left(s,\left(x_{\lambda}\right)_{s}\right) d s\right| \\
& \leq \frac{M}{\delta}\left\|\int_{s_{0}}^{s_{0}+T} F_{1}\left(s,\left(x_{\lambda}\right)_{s}\right) d s\right\|_{1} \leq \frac{M K}{\delta}\left\|\int_{s_{0}}^{s_{0}+T} F_{1}\left(s,\left(x_{\lambda}\right)_{s}\right) d s\right\| \\
& \leq \frac{M K}{\delta}\left(N+\zeta\left|x_{\lambda}\right|_{T}\right) .
\end{aligned}
$$

On the other hand, since

$$
x_{\lambda}(t)=x_{\lambda}\left(\xi_{0}\right)+\int_{\xi_{0}}^{t} \lambda f\left(s,\left(x_{\lambda}\right)_{s}\right) d s+\int_{\xi_{0}}^{t} \lambda g\left(s,\left(x_{\lambda}\right)_{s}\right) d u(s),
$$

conditions (i'), (v) and (3.18) hold, we conclude that

$$
\begin{aligned}
\left\|x_{\lambda}(t)\right\| & \leq\left\|x_{\lambda}\left(\xi_{0}\right)\right\|+\left\|\int_{\xi_{0}}^{t} f\left(s,\left(x_{\lambda}\right)_{s}\right) d s\right\|+\left\|\int_{\xi_{0}}^{t} g\left(s,\left(x_{\lambda}\right)_{s}\right) d u(s)\right\| \\
& \leq \frac{M K N}{\delta}+\left(\frac{M K}{\delta} \zeta+\eta\right)\left|x_{\lambda}\right|_{T}+N
\end{aligned}
$$

for all $t \in\left[s_{0}, s_{0}+T\right]$. Taking $\beta=\frac{M K N+N \delta}{\delta-M K \zeta-\delta \eta}$, we have the desired result.

Next, we exhibit two results for the case that $u$ is continuous on $\mathbb{R}$. 
Proposition 3.2.6. Under the hypotheses (H1)-(H4), assume that conditions (ii) of Proposition 3.2.2 and (i') of Proposition 3.2.4 hold. Moreover, assume that the following condition holds:

(vi) $u$ is continuous on $\mathbb{R}$, the map $t \mapsto f\left(t, x_{t}\right)$ is continuous on the interval $\left(s_{0}, s_{0}+T\right)$ whenever $x$ is continuous on $\mathbb{R}$ and there exists $0 \leq \eta<1$ such that

$$
\left\|\int_{t_{1}}^{t_{2}} f\left(s, x_{s}\right) d s\right\| \leq \eta|x|_{T}
$$

for all $t_{1}, t_{2} \in\left[s_{0}, s_{0}+T\right]$ and $x \in G_{T}^{-}\left(\mathbb{R}, \mathbb{R}^{n}\right)$.

Then, there exists a constant $\beta>0$ such that $\|x(t)\|<\beta$ for all $t \in \mathbb{R}$, whenever $x(t)$ is a $T$-periodic solution of (3.3), with $\lambda \in(0,1]$.

Proof. Let $x_{\lambda}(t)$ be an arbitrary $T$-periodic solution of (3.3) with $0<\lambda \leq 1$. Since $u$ is continuous on $\mathbb{R}$, it follows by Lemma 3.1.1 that $x_{\lambda}$ is also continuous on $\mathbb{R}$. Thus, by condition (vi), the map $t \mapsto f\left(t,\left(x_{\lambda}\right)_{t}\right)$ is continuous on particular on $\left(s_{0}, s_{0}+T\right)$. By the Mean Value Theorem for integrals on the interval $\left[s_{0}, s_{0}+T\right]$ (see Theorem 2.4.23), there exists $\xi \in\left(s_{0}, s_{0}+T\right)$ such that

$$
f_{j}\left(\xi,\left(x_{\lambda}\right) \xi\right)=\frac{1}{T} \int_{s_{0}}^{s_{0}+T} f_{j}\left(s,\left(x_{\lambda}\right)_{s}\right) d s
$$

where $j$ comes from condition (ii). As done in the proof of Proposition 3.2.5 (see (3.15)) we get

$$
\left\|\int_{s_{0}}^{s_{0}+T} f\left(s,\left(x_{\lambda}\right)_{s}\right) d s\right\| \leq N .
$$

Also, by the proof of Proposition 3.2.2, we conclude that $\left\|x_{\lambda}\left(\xi_{0}\right)\right\| \leq \frac{K^{\gamma} M N^{\gamma}}{T \gamma}$, where $k_{0} \in \mathbb{Z}$ is such that $\xi_{0}=\xi-r-k_{0} T \in\left[s_{0}, s_{0}+T\right]$. Using this last fact and conditions (i') and (vi), we obtain

$$
\begin{aligned}
\left\|x_{\lambda}(t)\right\| & \leq\left\|x_{\lambda}\left(\xi_{0}\right)\right\|+\left\|\int_{\xi_{0}}^{t} f\left(s,\left(x_{\lambda}\right)_{s}\right) d s\right\|+\left\|\int_{\xi_{0}}^{t} g\left(s,\left(x_{\lambda}\right)_{s}\right) d u(s)\right\| \\
& \leq \frac{K^{\gamma} M N^{\gamma}}{T^{\gamma}}+\eta\left|x_{\lambda}\right|_{T}+N
\end{aligned}
$$

for all $t \in\left[s_{0}, s_{0}+T\right]$. Consequently,

$$
\left|x_{\lambda}\right|_{T} \leq \frac{1}{1-\eta}\left(\frac{K^{\gamma} M N^{\gamma}}{T^{\gamma}}+N\right)=: \beta
$$

for an arbitrary $T$-periodic solution $x_{\lambda}(t)$ of $(3.3), \lambda \in(0,1]$.

Proposition 3.2.7. Under the hypotheses (H1)-(H4), assume that conditions (i') of Proposition 3.2.4 and (vi) of Proposition 3.2.6 are satisfied. In addition, suppose the following condition holds: 
(vii) there are $j \in\{1,2, \ldots, n\}, \gamma>0, M>0, r \in \mathbb{R}, d \geq 0$ and $\zeta \geq 0$ such that $\eta+\zeta<1$ and

$$
\|x(t-r)\| \leq M\left|f_{j}\left(t, x_{t}\right)\right|^{\gamma}+\zeta|x|_{T}+d
$$

for all $t \in\left[s_{0}, s_{0}+T\right]$ and $x \in G_{T}^{-}\left(\mathbb{R}, \mathbb{R}^{n}\right)$, where $f_{j}$ is the $j$ th coordinate of the function $f$.

Then, there exists a constant $\beta>0$ such that $\|x(t)\|<\beta$ for all $t \in \mathbb{R}$, whenever $x(t)$ is a $T$-periodic solution of (3.3), with $\lambda \in(0,1]$.

Proof. Let $x_{\lambda}(t)$ be an arbitrary $T$-periodic solution of (3.3) with $0<\lambda \leq 1$. Following the steps of the proof of Proposition 3.2.6, we obtain, using the Mean Value Theorem for integrals on the interval $\left[s_{0}, s_{0}+T\right]$, the existence of $\xi \in\left(s_{0}, s_{0}+T\right)$ such that

$$
f_{j}\left(\xi,\left(x_{\lambda}\right) \xi\right)=\frac{1}{T} \int_{s_{0}}^{s_{0}+T} f_{j}\left(s,\left(x_{\lambda}\right)_{s}\right) d s .
$$

Let $k_{0} \in \mathbb{Z}$ be such that $\xi_{0}=\xi-r-k_{0} T \in\left[s_{0}, s_{0}+T\right]$. Condition (vii) together with (3.19) imply that

$$
\begin{aligned}
\left\|x_{\lambda}\left(\xi_{0}\right)\right\| & =\left\|x_{\lambda}(\xi-r)\right\| \leq \frac{M}{T^{\gamma}}\left|\int_{s_{0}}^{s_{0}+T} f_{j}\left(s,\left(x_{\lambda}\right)_{s}\right) d s\right|^{\gamma}+\zeta\left|x_{\lambda}\right|_{T}+d \\
& \leq \frac{M K^{\gamma}}{T^{\gamma}}\left\|\int_{s_{0}}^{s_{0}+T} f\left(s,\left(x_{\lambda}\right)_{s}\right) d s\right\|^{\gamma}+\zeta\left|x_{\lambda}\right|_{T}+d \\
& \leq \frac{M K^{\gamma} N^{\gamma}}{T^{\gamma}}+\zeta\left|x_{\lambda}\right|_{T}+d,
\end{aligned}
$$

where we have used $\|\cdot\|_{1} \leq K\|\cdot\|$ in the second inequality and (3.15) in the last inequality, taking into account condition (i').

Now, using conditions (i') and (vi), and the estimate of $\left\|x_{\lambda}\left(\xi_{0}\right)\right\|$, we obtain

$$
\begin{aligned}
\left\|x_{\lambda}(t)\right\| & \leq\left\|x_{\lambda}\left(\xi_{0}\right)\right\|+\left\|\int_{\xi_{0}}^{t} f\left(s,\left(x_{\lambda}\right)_{s}\right) d s\right\|+\left\|\int_{\xi_{0}}^{t} g\left(s,\left(x_{\lambda}\right)_{s}\right) d u(s)\right\| \\
& \leq \frac{K^{\gamma} M N^{\gamma}}{T^{\gamma}}+\zeta\left|x_{\lambda}\right|_{T}+d+\eta\left|x_{\lambda}\right|_{T}+N
\end{aligned}
$$

for all $t \in\left[s_{0}, s_{0}+T\right]$. Then,

$$
\left|x_{\lambda}\right|_{T} \leq \frac{1}{1-\eta-\zeta}\left(\frac{K^{\gamma} M N^{\gamma}}{T^{\gamma}}+d+N\right)=: \beta,
$$

for an arbitrary $T$-periodic solution $x_{\lambda}(t)$ of (3.3), with $\lambda \in(0,1]$.

\subsection{Examples}

To end this chapter, we present some examples to illustrate the theory developed in the previous sections. The following examples are inserted in the general context of regulated functions and with integrals in the Perron sense. 
Example 3.3.1. Consider the measure FDE

$$
x(t)=x(0)-\int_{0}^{t}\left(\frac{n+\cos ^{2}(n s)}{a_{n}+\sin (n s)}\right) x^{2 m}(s-r) d s+\int_{0}^{t} \sin (s) d u(s),
$$

where $m \in \mathbb{N}, r \in \mathbb{R}, a_{n}>1, n \in \mathbb{N}, u(s)=s+\varepsilon \alpha(s)$ for all $s \in \mathbb{R}$, with $\varepsilon \in \mathbb{R}$, and $\alpha(s)=s-2 k \pi$ for all $s \in(2 \pi k, 2 \pi(k+1)], k \in \mathbb{Z}$.

Let $f, g: \mathbb{R} \times G^{-}(\mathbb{R}, \mathbb{R}) \rightarrow \mathbb{R}$ be defined by

$$
f(t, \phi)=\left(\frac{n+\cos ^{2}(n s)}{a_{n}+\sin (n s)}\right) \phi^{2 m}(-r) \quad \text { and } \quad g(t, \phi)=\sin (t),
$$

for all $t \in \mathbb{R}$ and all $\phi \in G^{-}(\mathbb{R}, \mathbb{R})$. Let us show that $u$ satisfies condition (H1) with $c=2 \pi$. Indeed, let $t \in \mathbb{R}$. Then $t \in(2 \pi k, 2 \pi(k+1)]$ for some $k \in \mathbb{Z}$. Since $t+2 \pi \in(2 \pi(k+1), 2 \pi(k+2)]$, we have

$$
\begin{aligned}
u(t+2 \pi) & =t+2 \pi+\varepsilon \alpha(t+2 \pi)=t+2 \pi+\varepsilon(t+2 \pi-2(k+1) \pi) \\
& =t+\varepsilon t-2 \pi k \varepsilon+2 \pi+2 \pi \varepsilon-2 \pi \varepsilon \\
& =t+\varepsilon(t-2 \pi k)+2 \pi=u(t)+c .
\end{aligned}
$$

Fix $s_{0} \in(0,2 \pi)$. Note that $u$ is continuous at $s_{0}$, since $u(t)=(1+\varepsilon) t$, for all $t \in(0,2 \pi]$. Therefore, (H1) holds. By Theorem 2.4.5, condition (H2) is also satisfied.

Let us verify that condition (H3) holds. Define $h: \mathbb{R} \rightarrow \mathbb{R}$ by $h(t)=t, t \in \mathbb{R}$. Note that

$$
\frac{n x^{2 m}(t-r)}{a_{n}+1} \leq\left(\frac{n+\cos ^{2}(n t)}{a_{n}+\sin (n t)}\right) x^{2 m}(t-r) \leq \frac{(n+1) x^{2 m}(t-r)}{a_{n}-1},
$$

for all $t \in \mathbb{R}$.

Given $\mu>0$, choose $N_{\mu}=1+|\varepsilon|+\frac{(n+1) \mu^{2 m}}{a_{n}-1}$. If $x \in G_{2 \pi}^{-}(\mathbb{R}, \mathbb{R})$ is such that $|x|_{T} \leq \mu$ and $t_{1}, t_{2} \in\left[s_{0}, s_{0}+2 \pi\right], t_{1} \leq t_{2}$, then it follows by (3.21) that

$$
\begin{aligned}
\left|\int_{t_{1}}^{t_{2}}\left(\frac{n+\cos ^{2}(n s)}{a_{n}+\sin (n s)}\right) x^{2 m}(s-r) d s\right| & \leq \int_{t_{1}}^{t_{2}}|x|_{T}^{2 m}\left(\frac{n+1}{a_{n}-1}\right) d s \\
& \leq \mu^{2 m}\left(\frac{n+1}{a_{n}-1}\right)\left|t_{2}-t_{1}\right| \\
& \leq N_{\mu}\left|t_{2}-t_{1}\right| .
\end{aligned}
$$

Also, if $t_{1}, t_{2} \in\left[s_{0}, s_{0}+2 \pi\right]$, then Theorem 2.4.5 guarantees that

$$
\int_{t_{1}}^{b} \sin (s) d u(s)=\lim _{\alpha \rightarrow t_{1}^{+}} \int_{\alpha}^{b} \sin (s) d u(s)+\sin \left(t_{1}\right) \Delta^{+} u\left(t_{1}\right)
$$

and

$$
\int_{b}^{t_{2}} \sin (s) d u(s)=\lim _{\beta \rightarrow t_{2}^{-}} \int_{b}^{\beta} \sin (s) d u(s)+\sin \left(t_{2}\right) \Delta^{-} u\left(t_{2}\right)
$$


Hence, by (3.22) and (3.23) we obtain

$$
\begin{aligned}
& \left|\int_{t_{1}}^{t_{2}} \sin (s) d u(s)\right|=\left|\int_{t_{1}}^{b} \sin (s) d u(s)+\int_{b}^{t_{2}} \sin (s) d u(s)\right| \\
& =\lim _{\substack{\alpha \rightarrow t_{1}^{+} \\
\beta \rightarrow t_{2}^{-}}}\left|\int_{\alpha}^{\beta} \sin (s) d u(s)+\sin \left(t_{2}\right) \Delta^{-} u\left(t_{2}\right)+\sin \left(t_{1}\right) \Delta^{+} u\left(t_{1}\right)\right| \\
& =\lim _{\substack{\alpha \rightarrow t_{1}^{+} \\
\beta \rightarrow t_{2}^{-}}}\left|\int_{\alpha}^{\beta} \sin (s) d u(s)\right| \leq \lim _{\substack{\alpha \rightarrow t_{1}^{+} \\
\beta \rightarrow t_{2}^{-}}}(1+|\varepsilon|)|\beta-\alpha| \\
& \leq N_{\mu}\left|t_{2}-t_{1}\right|,
\end{aligned}
$$

as $\Delta^{-} u(s)=\Delta^{+} u(s)=0$ for all $s \in\left[s_{0}, 2 \pi\right) \cup\left(2 \pi, s_{0}+2 \pi\right]$ and $\sin (2 \pi)=0$. This shows the validity of condition $(\mathrm{H} 3)$.

At last, since $g(t, \phi)=\sin (t)$, with $\phi \in G_{2 \pi}^{-}(\mathbb{R}, \mathbb{R})$ and $t \in \mathbb{R}$, does not depend on $\phi$ and

$$
\begin{aligned}
& \left|\int_{t_{1}}^{t_{2}} \frac{n+\cos ^{2}(n s)}{a_{n}+\sin (n s)}\left[x^{2 m}(s-r)-y^{2 m}(s-r)\right] d s\right| \leq \int_{t_{1}}^{t_{2}}\left(\frac{n+1}{a_{n}-1}\right)\left|x^{2 m}(s-r)-y^{2 m}(s-r)\right| d s \\
& \leq \int_{t_{1}}^{t_{2}}\left(\frac{n+1}{a_{n}-1}\right)\left|x^{2 m}-y^{2 m}\right|_{T} d s \leq \int_{t_{1}}^{t_{2}}\left(\frac{n+1}{a_{n}-1}\right) \sum_{k=0}^{2 m-1}|x|_{T}^{k}|y|_{T}^{2 m-1-k}|x-y|_{T} d s \\
& \leq \int_{t_{1}}^{t_{2}} 2 m \mu^{2 m-1}\left(\frac{n+1}{a_{n}-1}\right)|x-y|_{T} d s
\end{aligned}
$$

holds for all $x, y \in G_{2 \pi}^{-}(\mathbb{R}, \mathbb{R})$ such that $|x|_{T} \leq \mu$ and $|y|_{T} \leq \mu$, and for all $t_{1}, t_{2} \in\left[s_{0}, s_{0}+2 \pi\right]\left(t_{1} \leq\right.$ $\left.t_{2}\right)$, then condition (H4) is true with $M_{\mu}(s)=2 m \mu^{2 m-1}\left(\frac{n+1}{a_{n}-1}\right), s \in \mathbb{R}$, and with any non-negative locally Perron-Stieltjes integrable function $L_{\mu}: \mathbb{R} \rightarrow \mathbb{R}$ with respect to $u$.

In order to obtain a limiting factor for all possible $T$-periodic solutions of the measure FDE (3.20), we are going to show that conditions of Proposition 3.2.4 hold. By the previous calculus, we have

$$
\left|\int_{t_{1}}^{t_{2}} \sin (s) d u(s)\right| \leq(1+|\varepsilon|)\left|t_{2}-t_{1}\right| \leq 2 \pi(1+|\varepsilon|)
$$

for all $t_{1}, t_{2} \in\left[s_{0}, s_{0}+2 \pi\right]$, and since

$$
\begin{aligned}
\left|\int_{t_{1}}^{t_{2}}\left(\frac{n+\cos ^{2}(n s)}{a_{n}+\sin (n s)}\right) x^{2 m}(s-r) d s\right| & =\int_{t_{1}}^{t_{2}}\left(\frac{n+\cos ^{2}(n s)}{a_{n}+\sin (n s)}\right) x^{2 m}(s-r) d s \\
& \leq\left|\int_{s_{0}}^{s_{0}+2 \pi}\left(\frac{n+\cos ^{2}(n s)}{a_{n}+\sin (n s)}\right) x^{2 m}(s-r) d s\right|
\end{aligned}
$$

for all $t_{1}, t_{2} \in\left[s_{0}, s_{0}+2 \pi\right]$ and $x \in G_{2 \pi}^{-}(\mathbb{R}, \mathbb{R})$, it implies that conditions (i') and (ii') of Proposition 3.2.4 hold.

$$
\begin{aligned}
& \text { As } f\left(t, x_{t}\right)=-\left(\frac{n+\cos ^{2}(n t)}{a_{n}+\sin (n t)}\right) x^{2 m}(t-r) \text {, we have } \\
& \qquad|x(t-r)|^{2 m}=\left|\frac{a_{n}+\sin (n t)}{n+\cos ^{2}(n t)}\right|\left|f\left(t, x_{t}\right)\right| .
\end{aligned}
$$


This implies that

$$
|x(t-r)|=\left|\frac{a_{n}+\sin (n t)}{n+\cos ^{2}(n t)}\right|^{\frac{1}{2 m}}\left|f\left(t, x_{t}\right)\right|^{\frac{1}{2 m}} \leq\left(\frac{a_{n}+1}{n}\right)^{\frac{1}{2 m}} \mid f\left(t, x_{t}\right)^{\frac{1}{2 m}}
$$

for all $t \in\left[s_{0}, s_{0}+2 \pi\right]$ and all $x \in G_{2 \pi}^{-}(\mathbb{R}, \mathbb{R})$, from whence condition (ii) of Proposition 3.2.2 is valid.

Finally, note that $u$ is continuous on $\mathbb{R} \backslash\{2 k \pi: k \in \mathbb{Z}\}$ and, in particular, on $\left[s_{0}, s_{0}+\delta_{u}\right]$ with $0<\delta_{u}<2 \pi-s_{0}$. Let $s_{1} \in\left[s_{0}, s_{0}+\delta_{u}\right)$ be such that $s_{1}-r \neq 2 k \pi, k \in \mathbb{Z}$. Now, take $0<\delta<\delta_{u}+s_{0}-s_{1}$ such that $\left.\left[s_{1}-r, s_{1}-r+\delta\right] \subset\right] 2 q \pi, 2(q+1) \pi[$ for some $q \in \mathbb{Z}$. Thus, $f\left(t, x_{t}\right)=-\left(\frac{n+\cos ^{2}(n t)}{a_{n}+\sin (n t)}\right) x^{2 m}(t-r)$ is continuous on $\left[s_{1}, s_{1}+\delta\right]$ provided $x$ is continuous on $\Lambda_{u}$. Hence, condition (iii) of Proposition 3.2.2 holds.

By Proposition 3.2.4, there exists $\beta>0$ such that $|x(t)|<\beta$ for all $t \in \mathbb{R}$, whenever $x(t)$ is a $2 \pi$-periodic solution of the measure FDE

$$
x(t)=\lambda x(0)-\lambda \int_{0}^{t}\left(\frac{n+\cos ^{2}(n s)}{a_{n}+\sin (n s)}\right) x^{2 m}(s-r) d s+\lambda \int_{0}^{t} \sin (s) d u(s), \quad \lambda \in(0,1] .
$$

Therefore, by Theorem 3.1.9, the measure FDE (3.20) admits at least one $2 \pi$-periodic solution for each $n, m \in \mathbb{N}$.

Example 3.3.2. Consider the measure FDE

$$
\begin{gathered}
x(t)=x(0)+\int_{0}^{t} a(s) b\left(x\left(s-r_{1}\right)\right) d s+\int_{0}^{t}\left(\int_{-\infty}^{s} K(s, \xi) x(\xi) d \xi\right) d s \\
+\int_{0}^{t} p\left(s, x\left(s-r_{2}\right)\right) d u(s)
\end{gathered}
$$

satisfying the following conditions:

a) $r_{1}, r_{2} \in \mathbb{R}, u \in G^{-}(\mathbb{R}, \mathbb{R})$ is continuous on $\left[s_{0}, s_{0}+\delta_{u}\right], s_{0}<T$ and $\delta_{u}<T-s_{0}$, and there exists $c \in \mathbb{R}$ such that $u(t+T)=u(t)+c$ for all $t \in \mathbb{R}$;

b) $a, b \in G^{-}(\mathbb{R}, \mathbb{R})$ are continuous on $\left[s_{0}, s_{0}+\delta_{u}\right]$, there is $a_{0}>0$ such that $a_{0} \leq a(t)$ for all $t \in \mathbb{R}, b(0)=0$ and $b(s) \geq \rho|s|$ for all $s \in \mathbb{R}$, with $\rho>0$;

c) $a(t+T)=a(t)$ and $K(t+T, s+T)=K(t, s)$ for every $t, s \in \mathbb{R}$, with $T>0$;

d) for each $x \in G_{T}^{-}(\mathbb{R}, \mathbb{R})$, the maps $t \mapsto a(t) b\left(x\left(t-r_{1}\right)\right), t \mapsto K(\cdot, t) x(t)$, and $t \mapsto \int_{-\infty}^{t} K(t, s) x(s) d s$ are locally Perron integrable functions over $\mathbb{R}$;

e) there is a non-negative locally Perron integrable function $A: \mathbb{R} \rightarrow \mathbb{R}_{+}$such that, for all $t_{1}, t_{2} \in\left[s_{0}, s_{0}+T\right], t_{1} \leq t_{2}$,

$$
\left|\int_{t_{1}}^{t_{2}} a(s)\left[b\left(z_{1}\left(s-r_{1}\right)\right)-b\left(z_{2}\left(s-r_{1}\right)\right)\right] d s\right| \leq \int_{t_{1}}^{t_{2}}\left|z_{1}-z_{2}\right|_{T} A(s) d s,
$$

whenever $z_{1}, z_{2} \in G_{T}^{-}(\mathbb{R}, \mathbb{R})$; 
f) there exists a non-negative locally Perron integrable functions $Q: \mathbb{R} \rightarrow \mathbb{R}_{+}$such that $\left(\frac{1}{a_{0} \rho \delta_{u}}+1\right) \int_{s_{0}}^{s_{0}+T}[A(s)+Q(s)] d s<1$. Moreover, for $t_{1}, t_{2} \in\left[s_{0}, s_{0}+T\right], t_{1} \leq t_{2}$,

$$
\left|\int_{t_{1}}^{t_{2}}\left(\int_{-\infty}^{s} K(s, \xi)\left[z_{1}(\xi)-z_{2}(\xi)\right] d \xi\right) d s\right| \leq \int_{t_{1}}^{t_{2}}\left|z_{1}-z_{2}\right|_{T} Q(s) d s
$$

whenever $z_{1}, z_{2} \in G_{T}^{-}(\mathbb{R}, \mathbb{R})$;

g) $p$ is $T$-periodic in the first variable; for each $x \in G_{T}^{-}(\mathbb{R}, \mathbb{R})$, the mapping $t \mapsto p\left(t, x\left(t-r_{2}\right)\right)$ is locally Perron-Stieltjes integrable with respect to $u$ over $\mathbb{R}$;

h) there are non-negative locally Perron-Stieltjes integrable functions $P, N: \mathbb{R} \rightarrow \mathbb{R}_{+}$with respect to $u$ such that, for all $t_{1}, t_{2} \in\left[s_{0}, s_{0}+T\right], t_{1} \leq t_{2}$, we have

$$
\left|\int_{t_{1}}^{t_{2}} p\left(s, \phi\left(-r_{2}\right)\right) d u(s)\right| \leq \int_{t_{1}}^{t_{2}} P(s) d u(s)
$$

and

$$
\left|\int_{t_{1}}^{t_{2}}\left[p\left(s, \phi\left(-r_{2}\right)\right)-p\left(s, \psi\left(-r_{2}\right)\right)\right] d u(s)\right| \leq \int_{t_{1}}^{t_{2}} N(s)|\phi-\psi|_{T} d u(s),
$$

whenever $\phi, \psi \in G_{T}^{-}(\mathbb{R}, \mathbb{R})$.

Let $f, g: \mathbb{R} \times G^{-}(\mathbb{R}, \mathbb{R}) \rightarrow \mathbb{R}$ be defined by

$f(t, \phi)=a(t) b\left(\phi\left(-r_{1}\right)\right)+\int_{-\infty}^{t} K(t, \xi) \phi(\xi-t) d \xi \quad$ and $\quad g(t, \phi)=p\left(t, \phi\left(-r_{2}\right)\right)$,

for all $t \in \mathbb{R}$ and all $\phi \in G^{-}(\mathbb{R}, \mathbb{R})$. Note that (H1) follows from condition a). Condition (H2) follows immediately from the assumptions c), d) and $g$ ).

Set $h: \mathbb{R} \rightarrow \mathbb{R}$ by

$$
h(t)=\int_{0}^{t}[A(s)+Q(s)] d s+\int_{0}^{t} P(s) d u(s), \quad t \in \mathbb{R} .
$$

Note that $h \in G^{0-}(\mathbb{R}, \mathbb{R})$ (see Theorem 2.4.5). Given $\mu>0$, taking $N_{\mu}=\mu+1$, and using conditions e), f) and $\mathrm{h}$ ), we have for all $t_{1}, t_{2} \in\left[s_{0}, s_{0}+T\right], t_{1} \leq t_{2}$,

$$
\begin{aligned}
\left|\int_{t_{1}}^{t_{2}} f\left(s, x_{s}\right) d s\right| & \leq\left|\int_{t_{1}}^{t_{2}} a(s) b\left(x\left(s-r_{1}\right)\right) d s\right|+\left|\int_{t_{1}}^{t_{2}}\left(\int_{-\infty}^{s} K(s, \xi) x(\xi) d \xi\right) d s\right| \\
& \leq \int_{t_{1}}^{t_{2}} A(s)|x|_{T} d s+\int_{t_{1}}^{t_{2}} Q(s)|x|_{T} d s \\
& \leq \mu\left|h\left(t_{2}\right)-h\left(t_{1}\right)\right| \leq N_{\mu}\left|h\left(t_{2}\right)-h\left(t_{1}\right)\right|,
\end{aligned}
$$

whenever $x \in G_{T}^{-}(\mathbb{R}, \mathbb{R})$ is such that $|x|_{T} \leq \mu$. Also, for all $t_{1}, t_{2} \in\left[s_{0}, s_{0}+T\right], t_{1} \leq t_{2}$, we get

$$
\begin{aligned}
\left|\int_{t_{1}}^{t_{2}} g\left(s, x_{s}\right) d u(s)\right| & =\left|\int_{t_{1}}^{t_{2}} p\left(s, x\left(s-r_{2}\right)\right) d u(s)\right| \\
& \leq \int_{t_{1}}^{t_{2}} P(s) d u(s) \\
& \leq N_{\mu}\left|h\left(t_{2}\right)-h\left(t_{1}\right)\right|,
\end{aligned}
$$


whenever $x \in G_{T}^{-}(\mathbb{R}, \mathbb{R})$ is such that $|x|_{T} \leq \mu$. Therefore, we conclude that condition (H3) holds.

On the other hand, for $\mu>0$, the condition (H4) is true with the choices of $M_{\mu}(t)=$ $A(t)+Q(t)$ and $L_{\mu}(t)=N(t), t \in \mathbb{R}$. Indeed, by conditions e), f), for all $t_{1}, t_{2} \in\left[s_{0}, s_{0}+T\right]$, $t_{1} \leq t_{2}$

$$
\begin{aligned}
& \left|\int_{t_{1}}^{t_{2}}\left[f\left(s, x_{s}\right)-f\left(s, y_{s}\right)\right] d s\right| \leq \\
& \leq\left|\int_{t_{1}}^{t_{2}} a(s)\left[b\left(x\left(s-r_{1}\right)\right)-b\left(y\left(s-r_{1}\right)\right)\right] d s\right|+\left|\int_{t_{1}}^{t_{2}}\left(\int_{-\infty}^{s} K(s, \xi)[x(\xi)-y(\xi)] d \xi\right) d s\right| \\
& \leq \int_{t_{1}}^{t_{2}} A(s)|x-y|_{T} d s+\int_{t_{1}}^{t_{2}} Q(s)|x-y|_{T} d s \\
& =\int_{t_{1}}^{t_{2}} M_{\mu}(s)|x-y|_{T} d s
\end{aligned}
$$

whenever $x, y \in G_{T}^{-}(\mathbb{R}, \mathbb{R})$ are such that $|x|_{T} \leq \mu$ and $|y|_{T} \leq \mu$. Moreover, using condition $\mathrm{h}$ ), for all $t_{1}, t_{2} \in\left[s_{0}, s_{0}+T\right], t_{1} \leq t_{2}$, we get

$$
\begin{aligned}
& \left|\int_{t_{1}}^{t_{2}}\left[g\left(s, x_{s}\right)-g\left(s, y_{s}\right)\right] d u(s)\right| \leq \\
& \leq\left|\int_{t_{1}}^{t_{2}}\left[p\left(s, x\left(s-r_{2}\right)\right)-p\left(s, y\left(s-r_{2}\right)\right)\right] d u(s)\right| \leq \int_{t_{1}}^{t_{2}} N(s)|x-y|_{T} d u(s) \\
& =\int_{t_{1}}^{t_{2}} L_{\mu}(s)|x-y|_{T} d s,
\end{aligned}
$$

whenever $x, y \in G_{T}^{-}(\mathbb{R}, \mathbb{R})$ are such that $|x|_{T} \leq \mu$ and $|y|_{T} \leq \mu$.

Associated with the measure FDE (3.24), let us consider the family of measure FDEs

$$
\begin{gathered}
x(t)=\lambda x\left(s_{0}\right)+\lambda \int_{s_{0}}^{t} a(s) b\left(x\left(s-r_{1}\right)\right) d s+\lambda \int_{s_{0}}^{t}\left(\int_{-\infty}^{s} K(s, \xi) x(\xi) d \xi\right) d s \\
+\lambda \int_{s_{0}}^{t} p\left(s, x\left(s-r_{2}\right)\right) d u(s),
\end{gathered}
$$

for all $t \in \mathbb{R}$ and $0<\lambda \leq 1$.

In order to obtain a limiting factor for all possible $T$-periodic solutions of (3.25), we are going to show that the conditions of Proposition 3.2.5 hold.

Choosing $N=\int_{s_{0}}^{s_{0}+T} P(s) d u(s)<\infty$, it follows from h) that, for all $t_{1}, t_{2} \in\left[s_{0}, s_{0}+T\right]$ and $x \in G_{T}^{-}(\mathbb{R}, \mathbb{R})$,

$$
\left|\int_{t_{1}}^{t_{2}} p\left(s, x\left(s-r_{2}\right)\right) d u(s)\right| \leq \int_{t_{1}}^{t_{2}} P(s) d u(s) \leq N
$$

and so, the condition (i') of Proposition 3.2.4 holds.

Let $F_{1}, F_{2}: \mathbb{R} \times G^{-}(\mathbb{R}, \mathbb{R}) \rightarrow \mathbb{R}$ be defined by

$$
F_{1}(t, \phi)=a(t) b\left(\phi\left(-r_{1}\right)\right) \quad \text { and } \quad F_{2}(t, \phi)=\int_{-\infty}^{t} K(t, \xi) \phi(\xi-t) d \xi .
$$


By condition a), $u$ is continuous on $\left[s_{0}, s_{0}+\delta_{u}\right]$. Since $u \in G^{-}(\mathbb{R}, \mathbb{R})$, there exists $s_{1} \in\left(s_{0}, s_{0}+\delta_{u}\right)$ such that $s_{1}-r_{1}$ is a continuity point of $u$. Thus, we may choose $\delta \in\left(0, \delta_{u}+s_{0}-s_{1}\right)$ such that $u$ is continuous on $\left[s_{1}-r, s_{1}-r+\delta\right]$. Using condition $\left.\mathrm{b}\right)$, the map $t \mapsto F_{1}\left(t, x_{t}\right)$ is continuous on $\left[s_{1}, s_{1}+\delta\right]$, whenever $x$ is continuous on $\Lambda_{u}$. Further, using b), we have

$$
\rho a_{0}\left|x\left(t-r_{1}\right)\right| \leq a(t) b\left(x\left(t-r_{1}\right)\right)=F_{1}\left(t, x_{t}\right),
$$

that is,

$$
\left|x\left(t-r_{1}\right)\right| \leq \frac{1}{\rho a_{0}} F_{1}\left(t, x_{t}\right)
$$

for all $t \in \mathbb{R}$ and $x \in G_{T}^{-}(\mathbb{R}, \mathbb{R})$. This shows that condition (iv) of Proposition 3.2.5 holds.

Set $\eta=\int_{s_{0}}^{s_{0}+T}[A(s)+Q(s)] d s$, which satisfies $\eta \in[0,1)$ and $\left(\frac{1}{a_{0} \rho \delta_{u}}+1\right) \eta<1$ by virtue of condition f). Let $t_{1}, t_{2} \in\left[s_{0}, s_{0}+T\right], t_{1} \leq t_{2}$, and $x \in G_{T}^{-}(\mathbb{R}, \mathbb{R})$. Since

$$
\begin{gathered}
\left|\int_{t_{1}}^{t_{2}} a(s) b\left(x\left(s-r_{1}\right)\right) d s\right|+\left|\int_{t_{1}}^{t_{2}}\left(\int_{-\infty}^{s} K(s, \xi) x(\xi) d \xi\right) d s\right| \\
\leq \int_{t_{1}}^{t_{2}} A(s)|x|_{T} d s+\int_{t_{1}}^{t_{2}} Q(s)|x|_{T} d s \leq \eta|x|_{T},
\end{gathered}
$$

we conclude that

$$
\left|\int_{t_{1}}^{t_{2}} f\left(s, x_{s}\right) d s\right| \leq \eta|x|_{T} \quad \text { and } \quad\left|\int_{t_{1}}^{t_{2}} F_{2}\left(s, x_{s}\right) d s\right| \leq \eta|x|_{T} .
$$

Hence, condition (v) of Proposition 3.2.5 is verified.

In conclusion, Proposition 3.2.5 assures the existence of a priori bound for any $T$-periodic solution of the measure FDE (3.25), with $0<\lambda \leq 1$. Therefore, by Theorem 3.1.9, the measure FDE (3.24) admits at least one $T$-periodic solution.

Example 3.3.3. Consider the function

$$
f(t)= \begin{cases}2 t \sin \left(\frac{1}{t}\right)-\cos \left(\frac{1}{t}\right), & \text { if } 0<t \leq 2 \pi \\ 0, & \text { if } t=0\end{cases}
$$

The function $f$ is a Perron integrable function, but it is neither Riemann nor Lebesgue integrable (see the Example 2.4.24). Define a function $h: \mathbb{R} \rightarrow \mathbb{R}$ by

$$
h(t)=f(t-2 \pi k), \quad \text { for } \quad 2 \pi k<t \leq 2 \pi(k+1), \quad k \in \mathbb{Z} .
$$

Let $u: \mathbb{R} \rightarrow \mathbb{R}$ be given by

$$
u(t)=t-2 k \pi, \quad \text { for } \quad t \in(2 k \pi, 2(k+1) \pi], \quad k \in \mathbb{Z} .
$$

Consider the measure FDE

$$
x(t)=x(0)+\int_{0}^{t} \alpha_{1}\left|x\left(s-r_{1}\right)\right| d s+\int_{0}^{t}\left(\int_{-\infty}^{s} \alpha_{2} e^{-s+\xi} x(\xi) d \xi\right) d s
$$




$$
+\int_{0}^{t} h(s) \arctan \left[x\left(s-r_{2}\right)+1\right] d u(s), \quad t \in \mathbb{R}
$$

where $r_{1}, r_{2} \in \mathbb{R}$ and $\alpha_{1}, \alpha_{2}>0$ are such that $\left(\frac{1}{\alpha_{1} \delta_{u}}+1\right) 2 \pi\left(\alpha_{1}+\alpha_{2}\right)<1$. Let $s_{0} \in(0,2 \pi)$ and set $\delta_{u} \in\left(0,2 \pi-s_{0}\right)$.

Let us show that conditions a) $-\mathrm{h}$ ) from Example 3.3.2 hold. Here, we have $a(t)=1$, $b(t)=\alpha_{1}|t|, K(t, s)=\alpha_{2} e^{-t+s}$ and $p\left(t, x\left(t-r_{2}\right)\right)=h(t) \arctan \left[x\left(t-r_{2}\right)+1\right]$, for all $t, s \in \mathbb{R}$.

Since $u$ is continuous on $\left[s_{0}, 2 \pi\right)$, then $u$ is continuous on $\left[s_{0}, s_{0}+\delta_{u}\right]$. Note that $u$ is a $2 \pi$-periodic function, which implies that $u$ satisfies condition a) with $c=0$.

Choose $a_{0}=1$ and $\rho=\alpha_{1}$, so condition $\mathrm{b}$ ) is verified. Since $a$ is a constant function and

$$
K(t+2 \pi, s+2 \pi)=\alpha_{2} e^{-t-2 \pi+s+2 \pi}=\alpha_{2} e^{-t+s}=K(t, s),
$$

for all $t, s \in \mathbb{R}$, we have condition c) is true.

Let $x \in G_{2 \pi}^{-}(\mathbb{R}, \mathbb{R})$. For each $s \in \mathbb{R}$, the map $s \mapsto \alpha_{2} e^{-t+s} x(s)$ is locally Perron integrable function over $\mathbb{R}$. Also, the maps $t \mapsto \alpha_{1}\left|x\left(t-r_{1}\right)\right|$ and $t \mapsto \int_{-\infty}^{t} \alpha_{2} e^{-t+s} x(s) d s$ are locally Perron integrable functions over $\mathbb{R}$, because these maps are locally Lebesgue integrable functions over $\mathbb{R}$. Therefore, condition d) holds.

Condition e) is true with $A(t)=\alpha_{1}, t \in \mathbb{R}$. Indeed, let $t_{1}, t_{2} \in\left[s_{0}, s_{0}+2 \pi\right], t_{1} \leq t_{2}$, then

$$
\begin{aligned}
& \left|\int_{t_{1}}^{t_{2}} a(s)\left[b\left(x\left(s-r_{1}\right)\right)-b\left(y\left(s-r_{1}\right)\right)\right] d s\right|=\left|\int_{t_{1}}^{t_{2}} \alpha_{1}\left[\left|x\left(s-r_{1}\right)\right|-\left|y\left(s-r_{1}\right)\right|\right] d s\right| \\
& \leq \int_{t_{1}}^{t_{2}} \alpha_{1}|| x\left(s-r_{1}\right)|-| y\left(s-r_{1}\right)|| d s \leq \int_{t_{1}}^{t_{2}} \alpha_{1}\left|x\left(s-r_{1}\right)-y\left(s-r_{1}\right)\right| d s \\
& \leq \int_{t_{1}}^{t_{2}} A(s)|x-y|_{2 \pi} d s,
\end{aligned}
$$

whenever $x, y \in G_{2 \pi}^{-}(\mathbb{R}, \mathbb{R})$.

By the choices of $\alpha_{1}$ and $\alpha_{2}$, condition f) holds with $Q(t)=\alpha_{2}, t \in \mathbb{R}$. In fact,

$$
\begin{aligned}
\left(\frac{1}{a_{0} \rho \delta_{u}}+1\right) \int_{s_{0}}^{s_{0}+2 \pi}[A(s)+Q(s)] d s & =\left(\frac{1}{\alpha_{1} \delta_{u}}+1\right) \int_{s_{0}}^{s_{0}+2 \pi}\left[\alpha_{1}+\alpha_{2}\right] d s \\
& =\left(\frac{1}{\alpha_{1} \delta_{u}}+1\right) 2 \pi\left(\alpha_{1}+\alpha_{2}\right)<1
\end{aligned}
$$

and, for all $t_{1}, t_{2} \in\left[s_{0}, s_{0}+2 \pi\right], t_{1} \leq t_{2}$, and $x, y \in G_{2 \pi}^{-}(\mathbb{R}, \mathbb{R})$, we have

$$
\begin{aligned}
& \left|\int_{t_{1}}^{t_{2}}\left(\int_{-\infty}^{s} K(s, \xi)[x(\xi)-y(\xi)] d \xi\right) d s\right|=\left|\int_{t_{1}}^{t_{2}}\left(\int_{-\infty}^{s} \alpha_{2} e^{-s+\xi}[x(\xi)-y(\xi)] d \xi\right) d s\right| \\
& \leq \int_{t_{1}}^{t_{2}}\left|\int_{-\infty}^{s} \alpha_{2} e^{-s+\xi}[x(\xi)-y(\xi)] d \xi\right| d s \leq \int_{t_{1}}^{t_{2}}\left(\int_{-\infty}^{s} \alpha_{2} e^{-s+\xi}|x(\xi)-y(\xi)| d \xi\right) d s \\
& \leq \int_{t_{1}}^{t_{2}} \alpha_{2}\left(\int_{-\infty}^{s} e^{-s+\xi} d \xi\right)|x-y|_{2 \pi} d s=\int_{t_{1}}^{t_{2}}|x-y|_{2 \pi} Q(s) d s .
\end{aligned}
$$


Condition $\mathrm{g}$ ) holds as the function $p(t, \phi)=h(t) \arctan [\phi+1]$ is $2 \pi$-periodic in the first variable, and, the map $t \mapsto p\left(t, x\left(t-r_{2}\right)\right)$ is locally Perron-Stieltjes integrable with respect to $u$ over $\mathbb{R}$.

At last, we verify the validity of condition $\mathrm{h})$ with $P(t)=\left(4 \pi+2 s_{0}+1\right) \frac{\pi}{2}$ and $N(t)=$ $4 \pi+2 s_{0}+1$, for all $t \in \mathbb{R}$. Since $h$ is $2 \pi$-periodic, we have

$$
|h(\tau)|=\left|2 \tau \sin \left(\frac{1}{\tau}\right)-\cos \left(\frac{1}{\tau}\right)\right| \leq 2 \tau+1 \leq 2\left(s_{0}+2 \pi\right)+1,
$$

for all $\tau \in\left[s_{0}, s_{0}+2 \pi\right]$. Thus,

$$
\left|p\left(\tau, x\left(\tau-r_{2}\right)\right)\right|=|h(\tau)|\left|\arctan \left[x\left(\tau-r_{2}\right)+1\right]\right| \leq\left(2\left(s_{0}+2 \pi\right)+1\right) \frac{\pi}{2},
$$

for all $\tau \in \mathbb{R}$ and $x \in G_{2 \pi}^{-}(\mathbb{R}, \mathbb{R})$. Then, for all $t_{1}, t_{2} \in\left[s_{0}, s_{0}+2 \pi\right], t_{1} \leq t_{2}$, and $x \in G_{2 \pi}^{-}(\mathbb{R}, \mathbb{R})$, we have

$$
-\int_{t_{1}}^{t_{2}}\left(2\left(s_{0}+2 \pi\right)+1\right) \frac{\pi}{2} d u(s) \leq \int_{t_{1}}^{t_{2}} p\left(s, x\left(s-r_{2}\right)\right) d u(s) \leq \int_{t_{1}}^{t_{2}}\left(2\left(s_{0}+2 \pi\right)+1\right) \frac{\pi}{2} d u(s),
$$

that is,

$$
\left|\int_{t_{1}}^{t_{2}} p\left(s, x\left(s-r_{2}\right)\right) d u(s)\right| \leq \int_{t_{1}}^{t_{2}}\left(4 \pi+2 s_{0}+1\right) \frac{\pi}{2} d u(s) .
$$

Moreover, for all $\tau \in \mathbb{R}$ and $x, y \in G_{2 \pi}^{-}(\mathbb{R}, \mathbb{R})$, we obtain

$$
\begin{aligned}
& \left|p\left(\tau, x\left(\tau-r_{2}\right)\right)-p\left(\tau, y\left(\tau-r_{2}\right)\right)\right| \\
& =|h(\tau)|\left|\arctan \left[x\left(\tau-r_{2}\right)+1\right]-\arctan \left[y\left(\tau-r_{2}\right)+1\right]\right| \\
& \leq\left(2\left(s_{0}+2 \pi\right)+1\right)|x-y|_{2 \pi} .
\end{aligned}
$$

Consequently,

$$
\left|\int_{t_{1}}^{t_{2}}\left[p\left(s, x\left(s-r_{2}\right)\right)-p\left(s, y\left(s-r_{2}\right)\right)\right] d u(s)\right| \leq \int_{t_{1}}^{t_{2}} N(s)|x-y|_{2 \pi} d u(s),
$$

for all $t_{1}, t_{2} \in\left[s_{0}, s_{0}+2 \pi\right]$ and $x, y \in G_{2 \pi}^{-}(\mathbb{R}, \mathbb{R})$. Therefore, it follows by Example 3.3.2 that the measure FDE (5.20) admits a $2 \pi$-periodic solution. 

CHAPTER

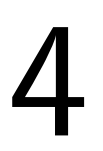

IMPULSIVE FUNCTIONAL DIFFERENTIAL

EQUATIONS

This chapter is dedicated to the study of periodic solutions for impulsive functional differential equations (impulsive FDEs for short) of type

$$
\left\{\begin{array}{l}
\dot{x}(t)=F\left(t, x_{t}\right), \quad t \neq t_{k}, \\
\Delta^{+} x\left(t_{k}\right)=I_{k}\left(x\left(t_{k}\right)\right),
\end{array} \quad k \in \mathbb{Z},\right.
$$

where the moments of impulse effects $\left\{\ldots, t_{-k}, \ldots, t_{-1}, t_{0}, t_{1}, \ldots, t_{k}, \ldots\right\} \subset \mathbb{R}$ satisfy

$$
\ldots<t_{-k}<\ldots<t_{-2}<t_{-1}<t_{0}<t_{1}<t_{2}<\ldots<t_{k}<\ldots
$$

and $\lim _{k \rightarrow \pm \infty} t_{k}= \pm \infty$

The existence result of periodic solutions of the impulsive FDE (4.1) is obtained by means of the periodicity theory of measure functional differential equations presented in Section 3.1, Chapter 3.

\subsection{Existence of periodic solutions}

In order to guarantee the existence of periodic solutions for the impulsive FDE (4.1), we shall assume throughout this section the following general conditions:

(B1) there exist $T>0$ and $q \in \mathbb{Z}_{+}$such that $0<t_{0}<t_{1}<\cdots<t_{q-1}<T$ and $t_{k+q}=t_{k}+T$, $k \in \mathbb{Z}$

(B2) $I_{k+q}=I_{k}$ for all $k \in \mathbb{Z}$;

(B3) $F: \mathbb{R} \times G^{-}\left(\mathbb{R}, \mathbb{R}^{n}\right) \rightarrow \mathbb{R}^{n}$ is $T$-periodic with respect to the first variable and, for each $x \in G_{T}^{-}\left(\mathbb{R}, \mathbb{R}^{n}\right)$, the map $t \mapsto F\left(t, x_{t}\right)$ is locally Perron integrable over $\mathbb{R}$; 
(B4) the impulse operators $I_{k}: \mathbb{R}^{n} \rightarrow \mathbb{R}^{n}, k \in \mathbb{Z}$, are continuous and there are $\tilde{M}_{1}, \tilde{M}_{2}>0$ such that $\left\|I_{k}(x)\right\| \leq \tilde{M}_{1}$ and $\left\|I_{k}(x)-I_{k}(y)\right\| \leq \tilde{M}_{2}\|x-y\|$ for all $x, y \in \mathbb{R}^{n}$ and $k \in \mathbb{Z}$;

(B5) there is a non-negative locally Perron integrable function $P: \mathbb{R} \rightarrow \mathbb{R}$ such that for a given $\mu>0$ one can obtain a constant $\tilde{N}_{\mu}>0$ such that for all $s_{1}, s_{2} \in[0, T], s_{1} \leq s_{2}$,

$$
\left\|\int_{s_{1}}^{s_{2}} F\left(t, x_{t}\right) d t\right\| \leq \int_{s_{1}}^{s_{2}} \tilde{N}_{\mu} P(t) d t
$$

whenever $x \in G_{T}^{-}\left(\mathbb{R}, \mathbb{R}^{n}\right)$ and $|x|_{T} \leq \mu$;

(B6) given $\mu>0$, there exists a non-negative locally Perron integrable function $C_{\mu}: \mathbb{R} \rightarrow \mathbb{R}$ such that for all $s_{1}, s_{2} \in[0, T], s_{1} \leq s_{2}$,

$$
\left\|\int_{s_{1}}^{s_{2}}\left[F\left(t, x_{t}\right)-F\left(t, y_{t}\right)\right] d t\right\| \leq \int_{s_{1}}^{s_{2}} C_{\mu}(t)|x-y|_{T} d t,
$$

for all $x, y \in G_{T}^{-}\left(\mathbb{R}, \mathbb{R}^{n}\right)$ such that $|x|_{T} \leq \mu$ and $|y|_{T} \leq \mu$.

Definition 4.1.1. A function $x: \mathbb{R} \rightarrow \mathbb{R}^{n}$ is said to be a solution of the impulsive FDE (4.1), if the following conditions hold:

(i) $x \in G^{-}\left(\left(t_{k}, t_{k+1}\right], \mathbb{R}^{n}\right)$ for all $k \in \mathbb{Z}$;

(ii) $\dot{x}(t)=F\left(t, x_{t}\right)$ almost everywhere in $\mathbb{R} \backslash\left\{t_{k}: k \in \mathbb{Z}\right\}$;

(iii) $x\left(t_{k}^{+}\right)-x\left(t_{k}\right)=I_{k}\left(x\left(t_{k}\right)\right), k \in \mathbb{Z}$.

Recall that a function $x$ is a solution of the impulsive FDE (4.1) if and only if $x$ satisfies the following integral equation

$$
x(t)=x(0)+\int_{0}^{t} F\left(s, x_{s}\right) d s+\Gamma(t), \quad t \in \mathbb{R},
$$

where

$$
\Gamma(t)= \begin{cases}\sum_{0<t_{k}<t} I_{k}\left(x\left(t_{k}\right)\right), & \text { if } t \geq 0, \\ -\sum_{t \leq t_{k}<0} I_{k}\left(x\left(t_{k}\right)\right), & \text { if } t<0 .\end{cases}
$$

In this way, our problem consists to find periodic solutions of the integral equation (4.2). Through Lemma 2.4.10, presented in Chapter 2, we can obtain a correspondence result between the solutions of the impulsive integral equation (4.2) and its corresponding measure FDE. See Lemma 4.1 .2 below.

Lemma 4.1.2. A function $x: \mathbb{R} \rightarrow \mathbb{R}^{n}$ is a solution of the integral equation (4.2) if and only if $x$ is a solution of the following measure FDE

$$
x(t)=x(0)+\int_{0}^{t} \tilde{f}\left(s, x_{s}\right) d \tilde{u}(s), \quad t \in \mathbb{R}
$$


where $\tilde{f}: \mathbb{R} \times G^{-}\left(\mathbb{R}, \mathbb{R}^{n}\right) \rightarrow \mathbb{R}^{n}$ is given by

$$
\tilde{f}\left(t, x_{t}\right)=\left\{\begin{array}{lll}
F\left(t, x_{t}\right), & t \neq t_{k}, & k \in \mathbb{Z}, \\
I_{k}(x(t)), & t=t_{k}, & k \in \mathbb{Z},
\end{array}\right.
$$

and $\tilde{u}: \mathbb{R} \rightarrow \mathbb{R}$ by

$$
\tilde{u}(t)=t+k, \quad t \in\left(t_{k}, t_{k+1}\right], \quad k \in \mathbb{Z} .
$$

Proof. By the definition of $\tilde{u}$, we have

$$
\Delta^{+} \tilde{u}\left(t_{k}\right)=\tilde{u}\left(t_{k}^{+}\right)-\tilde{u}\left(t_{k}\right)=t_{k}+k-t_{k}-(k-1)=1, \text { for all } k \in \mathbb{Z} .
$$

Using Lemma 2.4.10, if $t \geq 0$ then

$$
\begin{aligned}
\int_{0}^{t} \tilde{f}\left(s, x_{s}\right) d \tilde{u}(s) & =\int_{0}^{t} F\left(s, x_{s}\right) d s+\sum_{0<t_{k}<t} \tilde{f}\left(t_{k}, x_{t_{k}}\right) \Delta^{+} \tilde{u}\left(r_{k}\right) \\
& =\int_{0}^{t} F\left(s, x_{s}\right) d s+\sum_{0<t_{k}<t} I_{k}\left(x\left(t_{k}\right)\right) .
\end{aligned}
$$

Similarly, if $t<0$ then

$$
\begin{aligned}
-\int_{0}^{t} \tilde{f}\left(s, x_{s}\right) d \tilde{u}(s) & =\int_{t}^{0} \tilde{f}\left(s, x_{s}\right) d \tilde{u}(s)=\int_{t}^{0} F\left(s, x_{s}\right) d s+\sum_{t \leq t_{k}<0} \tilde{f}\left(t_{k}, x_{t_{k}}\right) \Delta^{+} \tilde{u}\left(r_{k}\right) \\
& =-\int_{0}^{t} F\left(s, x_{s}\right) d s+\sum_{t \leq t_{k}<0} I_{k}\left(x\left(t_{k}\right)\right),
\end{aligned}
$$

that is,

$$
\int_{0}^{t} \tilde{f}\left(s, x_{s}\right) d \tilde{u}(s)=\int_{0}^{t} F\left(s, x_{s}\right) d s-\sum_{t \leq t_{k}<0} I_{k}\left(x\left(t_{k}\right)\right) .
$$

Therefore, $x: \mathbb{R} \rightarrow \mathbb{R}^{n}$ is a solution of the integral equation (4.2) if and only if $x$ is a solution of the measure FDE (4.4).

Since $t_{-1}<0<t_{0}$, we have $\tilde{u}$ is continuous at $s_{0}=0$. Thus, associated to the measure FDE (4.4) presented in Lemma 4.1.2, we shall consider the following family of measure FDEs

$$
x(t)=\lambda x(0)+\lambda \int_{0}^{t} \tilde{f}\left(s, x_{s}\right) d \tilde{u}(s), \quad t \in \mathbb{R}, \quad \lambda \in(0,1] .
$$

Remark 4.1.3. By the proof of Lemma 4.1.2, we conclude that a function $x: \mathbb{R} \rightarrow \mathbb{R}^{n}$ is a solution of the integral equation

$$
x(t)=\lambda x(0)+\int_{0}^{t} \lambda F\left(s, x_{s}\right) d s+\lambda \Gamma(t), \quad t \in \mathbb{R}, \quad \lambda \in(0,1],
$$

if and only if $x$ is solution of (4.7), where $\Gamma(t)$ comes from (4.3).

Lemma 4.1.4. Let $\tilde{f}$ and $\tilde{u}$ be the functions given by (4.5) and (4.6), respectively. Assume that conditions (B1)-(B6) hold. Then, conditions (H1)-(H4) are satisfied, with $s_{0}=0, g=\tilde{f}, u=\tilde{u}$ and $f=0$. 
Proof. Since $\tilde{u}(t)=t+k$ for all $t \in\left(t_{k}, t_{k+1}\right], k \in \mathbb{Z}$, we have $\tilde{u}$ is left-continuous and locally of bounded variation in $\mathbb{R}$. Moreover, given $t \in \mathbb{R}$ there exists $k \in \mathbb{Z}$ such that $t_{k}<t \leq t_{k+1}$. Using condition (B1), we get $t_{k+q}<t+T \leq t_{k+q+1}$. Thus,

$$
\tilde{u}(t+T)=t+T+k+q=\tilde{u}(t)+q+T
$$

and condition (H1) holds with $c=T+q$.

According to conditions (B1)-(B3), the function $\tilde{f}: \mathbb{R} \times G^{-}\left(\mathbb{R}, \mathbb{R}^{n}\right) \rightarrow \mathbb{R}^{n}$ is $T$-periodic with respect to the first variable and, by Lemma 2.4.10, the map $t \mapsto \tilde{f}\left(t, x_{t}\right)$ is locally PerronStieltjes integrable over $\mathbb{R}$ with respect to $\tilde{u}$, whenever $x \in G_{T}^{-}\left(\mathbb{R}, \mathbb{R}^{n}\right)$. Thus, we conclude that condition $(\mathrm{H} 2)$ is true.

Now, let us check that condition (H3) is satisfied. Define $\tilde{P}: \mathbb{R} \rightarrow \mathbb{R}$ by

$$
\tilde{P}(t)=\left\{\begin{array}{lll}
P(t), & t \neq t_{k}, & k \in \mathbb{Z}, \\
\tilde{M}_{1}, & t=t_{k}, & k \in \mathbb{Z},
\end{array}\right.
$$

where the function $P$ comes from condition (B5) and $\tilde{M}_{1}$ comes from condition (B4). Let $h \in G^{-}(\mathbb{R}, \mathbb{R})$ be given by

$$
h(t)=t+\int_{0}^{t} \tilde{P}(s) d \tilde{u}(s), \quad t \in \mathbb{R} .
$$

Note that $h$ is continuous at $s_{0}=0$ since $\tilde{u}$ is continuous at this point (see Theorem 2.4.5). Given $\mu>0$ arbitrary, set $N_{\mu}=\max \left\{\tilde{N}_{\mu}, 1\right\}$, where $\tilde{N}_{\mu}$ comes from (B5). Let $s_{1} \leq s_{2}, s_{1}, s_{2} \in[0, T]$ and $x \in G_{T}^{-}\left(\mathbb{R}, \mathbb{R}^{n}\right)$ with $|x|_{T} \leq \mu$. Using (B4) and (B5), we get

$$
\begin{aligned}
\left\|\int_{s_{1}}^{s_{2}} \tilde{f}\left(t, x_{t}\right) d \tilde{u}(t)\right\| & \leq\left\|\int_{s_{1}}^{s_{2}} F\left(t, x_{t}\right) d t\right\|+\sum_{s_{1} \leq t_{k}<s_{2}}\left\|I_{k}\left(x\left(t_{k}\right)\right)\right\| \\
& \leq \int_{s_{1}}^{s_{2}} \tilde{N}_{\mu} P(t) d t+\sum_{s_{1} \leq t_{k}<s_{2}} \tilde{M}_{1} \\
& \leq N_{\mu} \int_{s_{1}}^{s_{2}} \tilde{P}(t) d \tilde{u}(t) \\
& \leq N_{\mu}\left|h\left(s_{2}\right)-h\left(s_{1}\right)\right| .
\end{aligned}
$$

Lastly, we verify that condition (H4) holds. Given $\mu>0$ arbitrary, set

$$
M_{\mu}(t)=\left\{\begin{array}{lll}
C_{\mu}(t), & t \neq t_{k}, & k \in \mathbb{Z} \\
\tilde{M}_{2}, & t=t_{k}, & k \in \mathbb{Z}
\end{array}\right.
$$

with $t \in \mathbb{R}$, where $\tilde{M}_{2}$ comes from condition (B4) and $C_{\mu}(t)$ comes from (B6). Let $s_{1} \leq s_{2}$, $s_{1}, s_{2} \in[0, T]$, and $x, y \in G_{T}^{-}\left(\mathbb{R}, \mathbb{R}^{n}\right)$ be such that $|x|_{T} \leq \mu$ and $|y|_{T} \leq \mu$. Then, using (B4) and (B6), we have 


$$
\begin{aligned}
\left\|\int_{s_{1}}^{s_{2}}\left[\tilde{f}\left(t, x_{t}\right)-\tilde{f}\left(t, y_{t}\right)\right] d \tilde{u}(t)\right\| & \leq\left\|\int_{s_{1}}^{s_{2}}\left[F\left(t, x_{t}\right)-F\left(t, y_{t}\right)\right] d t\right\|+\sum_{s_{1} \leq t_{k}<s_{2}}\left\|I_{k}\left(x\left(t_{k}\right)\right)-I_{k}\left(y\left(t_{k}\right)\right)\right\| \\
& \leq \int_{s_{1}}^{s_{2}} C_{\mu}(t)|x-y|_{T} d t+\sum_{s_{1} \leq t_{k}<s_{2}} \tilde{M}_{2}|x-y|_{T} \\
& =\int_{s_{1}}^{s_{2}} M_{\mu}(t)|x-y|_{T} d \tilde{u}(t) .
\end{aligned}
$$

Therefore, conditions (H1)-(H4) are satisfied.

Theorem 4.1.5. Assume that conditions (B1)-(B6) hold. If there exists a constant $\beta>0$ such that $\|x(t)\|<\beta$ for all $t \in \mathbb{R}$, whenever $x(t)$ is a $T$-periodic solution of (4.8), with $\lambda \in(0,1]$, then the impulsive FDE (4.1) has at least one $T$-periodic solution.

Proof. By Lemma 4.1.4, conditions (H1)-(H4) are satisfied with $s_{0}=0, g=\tilde{f}, u=\tilde{u}$ and $f=0$. By Remark 4.1.3, $\|x(t)\|<\beta$ for all $t \in \mathbb{R}$, whenever $x(t)$ is a $T$-periodic solution of the family

$$
x(t)=\lambda x(0)+\lambda \int_{0}^{t} \tilde{f}\left(s, x_{s}\right) d \tilde{u}(s), \quad t \in \mathbb{R}, \quad \lambda \in(0,1] .
$$

Consequently, by Theorem 3.1.9, the measure FDE

$$
x(t)=x(0)+\int_{0}^{t} \tilde{f}\left(s, x_{s}\right) d \tilde{u}(s), \quad t \in \mathbb{R},
$$

admits at least one $T$-periodic solution. Therefore, according to Lemma 4.1.2, the integral equation (4.2) has at least one $T$-periodic solution, that is, the the impulsive FDE (4.1) has at least one $T$-periodic solution.

\subsection{An example}

Consider the impulsive nonlinear Volterra integrodifferential equation with delay

$$
\left\{\begin{array}{l}
\dot{x}(t)=a(t) h(x(t))+\int_{t-r}^{t} B(t, s) g(x(s)) d s+p(t), \quad t \neq t_{k}, k \in \mathbb{Z} \\
x\left(t_{k}^{+}\right)-x\left(t_{k}\right)=b_{k}
\end{array}\right.
$$

under the following general conditions:

a) $r \in \mathbb{R}, a \in G_{T}^{-}\left(\mathbb{R}, \mathbb{R}_{+}\right), h \in C_{T}\left(\mathbb{R}, \mathbb{R}_{+}\right), B \in G_{T}^{-}\left(\mathbb{R}^{2},(0, \infty)\right)$, and there are constants $C, a_{0}>$ 0 such that $|B(t, s)| \leq C$ and $a(t) \geq a_{0}$, for all $t, s \in \mathbb{R}$;

b) there exist $T>0$ and $q \in \mathbb{Z}_{+}$such that $0<t_{0}<t_{1}<\ldots<t_{q-1}<T, t_{k+q}=t_{k}+T, b_{k} \in \mathbb{R}$ and $b_{q+k}=b_{k}$, for all $k \in \mathbb{Z}$. In addition, assume that there is a constant $M>0$ such that $\left|b_{k}\right| \leq M$, for all $k \in \mathbb{Z}$; 
c) $g: \mathbb{R} \rightarrow \mathbb{R}_{+}$and $p: \mathbb{R} \rightarrow \mathbb{R}$ are $T$-periodic functions; for each $x \in G_{T}^{-}(\mathbb{R}, \mathbb{R})$, the mappings $t \mapsto p(t)$ and $t \mapsto a(t) h(x(t))$ are locally Perron integrable over $\mathbb{R}$ and $t \mapsto g(x(t))$ is locally Lebesgue integrable over $\mathbb{R}$;

d) there is a function $R_{g}: \mathbb{R} \rightarrow \mathbb{R}_{+}$such that

$$
|g(x(t))-g(y(t))| \leq R_{g}(t)|x-y|_{T}
$$

for all $x, y \in G_{T}^{-}(\mathbb{R}, \mathbb{R})$ and $t \in \mathbb{R}$. In addition, there is a locally Lebesgue integrable function $L_{g}: \mathbb{R} \rightarrow \mathbb{R}_{+}$such that, for all $x \in G_{T}^{-}(\mathbb{R}, \mathbb{R})$ and $s_{1}, s_{2} \in \mathbb{R}, s_{1} \leq s_{2}$,

$$
\left|\int_{s_{1}}^{s_{2}} g(x(t)) d t\right| \leq \int_{s_{1}}^{s_{2}} L_{g}(t) d t
$$

e) given $J>0$, there exists $A>0$ such that

$$
\frac{h(s)}{|s|} \geq J \quad \text { whenever } \quad|s| \geq A, s \in \mathbb{R}
$$

f) there are locally Perron integrable functions $Q, \tilde{P}: \mathbb{R} \rightarrow \mathbb{R}_{+}$such that, for all $x \in G_{T}^{-}(\mathbb{R}, \mathbb{R})$ and $s_{1}, s_{2} \in[0, T], s_{1} \leq s_{2}$,

$$
\left|\int_{s_{1}}^{s_{2}} a(t) h(x(t)) d t\right| \leq \int_{s_{1}}^{s_{2}} Q(t)|x|_{T} d t
$$

and

$$
\left|\int_{s_{1}}^{s_{2}} p(t) d t\right| \leq \int_{s_{1}}^{s_{2}} \tilde{P}(t) d t
$$

g) there is a locally Perron integrable function $N: \mathbb{R} \rightarrow \mathbb{R}_{+}$such that, for all $s_{1}, s_{2} \in[0, T]$, $s_{1} \leq s_{2}$,

$$
\left|\int_{s_{1}}^{s_{2}} a(t)[h(x(t))-h(y(t))] d t\right| \leq \int_{s_{1}}^{s_{2}} N(t)|x-y|_{T} d t
$$

whenever $x, y \in G_{T}^{-}(\mathbb{R}, \mathbb{R})$.

Let us show that conditions (B1)-(B6) hold. In fact, note that condition (B1) follows from condition b). Now, define $I_{k}: \mathbb{R} \rightarrow \mathbb{R}$ by $I_{k}(x)=b_{k}$ for all $x \in \mathbb{R}$ and $k \in \mathbb{Z}$. Thus, conditions a) and b) imply conditions (B1), (B2) and (B4).

Define $F: \mathbb{R} \times G^{-}(\mathbb{R}, \mathbb{R}) \rightarrow \mathbb{R}$ by

$$
F(t, \varphi)=a(t) h(\varphi(0))+\int_{t-r}^{t} B(t, s) g(\varphi(s-t)) d s+p(t) .
$$

By a) and c), we have $F$ is $T$-periodic with respect to the first variable.

Condition c) and the boundedness of $B$ shows that $t \mapsto F\left(t, x_{t}\right)$ is locally Perron integrable over $\mathbb{R}$ for each $x \in G_{T}^{-}(\mathbb{R}, \mathbb{R})$. Hence, condition (B3) holds. 
Let us verify condition (B5). Let $\mu>0$ be arbitrary and define $\tilde{N}_{\mu}=\max \{\mu, C, 1\}$, where $C$ comes from condition a). Given $x \in G_{T}^{-}(\mathbb{R}, \mathbb{R})$ with $|x|_{T} \leq \mu$ and using d) and f), we have for all $s_{1}, s_{2} \in[0, T], s_{1} \leq s_{2}$,

$$
\begin{aligned}
& \left|\int_{s_{1}}^{s_{2}} F\left(t, x_{t}\right) d t\right| \leq \\
& \leq\left|\int_{s_{1}}^{s_{2}} a(t) h(x(t)) d t\right|+\left|\int_{s_{1}}^{s_{2}}\left(\int_{t-r}^{t} B(t, s) g(x(s)) d s\right) d t\right|+\left|\int_{s_{1}}^{s_{2}} p(t) d t\right| \\
& \leq \int_{s_{1}}^{s_{2}} Q(t)|x|_{T} d t+\int_{s_{1}}^{s_{2}}\left(\int_{t-r}^{t} B(t, s) g(x(s)) d s\right) d t+\int_{s_{1}}^{s_{2}} \tilde{P}(t) d t \\
& \leq \int_{s_{1}}^{s_{2}} Q(t) \mu d t+C \int_{s_{1}}^{s_{2}}\left(\int_{t-r}^{t} L_{g}(s) d s\right) d t+\int_{s_{1}}^{s_{2}} \tilde{P}(t) d t \\
& \leq \tilde{N}_{\mu} \int_{s_{1}}^{s_{2}} P(t) d t
\end{aligned}
$$

where $P(t)=Q(t)+\int_{t-r}^{t} L_{g}(s) d s+\tilde{P}(t), t \in \mathbb{R}$.

Lastly, we verify condition (B6). Let $\mu>0$ be arbitrary. Given $x, y \in G_{T}^{-}(\mathbb{R}, \mathbb{R})$ with $|x|_{T} \leq \mu,|y|_{T} \leq \mu$ and using d) and g), we have for all $s_{1}, s_{2} \in[0, T], s_{1} \leq s_{2}$,

$$
\begin{aligned}
& \left|\int_{s_{1}}^{s_{2}}\left[F\left(t, x_{t}\right)-F\left(t, y_{t}\right)\right] d t\right| \leq\left|\int_{s_{1}}^{s_{2}} a(t)[h(x(t))-h(y(t))] d t\right| \\
& +\left|\int_{s_{1}}^{s_{2}}\left(\int_{t-r}^{t} B(t, s)[g(x(s))-g(y(s))] d s\right) d t\right| \leq \int_{s_{1}}^{s_{2}} N(t)|x-y|_{T} d t \\
& +\int_{s_{1}}^{s_{2}}\left(-\int_{r}^{0}|B(t, t-\tau)||g(x(t-\tau))-g(y(t-\tau))| d \tau\right) d t \\
& \leq \int_{s_{1}}^{s_{2}} N(t)|x-y|_{T} d t+\int_{s_{1}}^{s_{2}}\left(\int_{0}^{r} C R_{g}(t-\tau) d \tau\right)|x-y|_{T} d t \\
& =\int_{s_{1}}^{s_{2}} C_{\mu}(t)|x-y|_{T} d t
\end{aligned}
$$

where $C_{\mu}(t)=N(t)+\int_{0}^{r} C R_{g}(t-\tau) d \tau, t \in \mathbb{R}$.

Now, let us consider the following family

$$
x(t)=\lambda x(0)+\int_{0}^{t} \lambda F\left(s, x_{s}\right) d s+\lambda \Gamma_{1}(t), \quad t \in \mathbb{R}, \quad \lambda \in(0,1],
$$

where

$$
\Gamma_{1}(t)= \begin{cases}\sum_{0<t_{k}<t} b_{k}, & \text { if } t \geq 0 \\ -\sum_{t \leq t_{k}<0} b_{k}, & \text { if } t<0\end{cases}
$$

Let $x_{\lambda}(t)$ be an arbitrary $T$-periodic solution of (4.10), with $0<\lambda \leq 1$. By Remark 4.1.3, $x_{\lambda}(t)$ is solution of the following measure FDE

$$
x(t)=\lambda x(0)+\lambda \int_{0}^{t} \tilde{f}\left(s, x_{s}\right) d \tilde{u}(s), \quad t \in \mathbb{R},
$$


with $0<\lambda \leq 1$, where $\tilde{f}: \mathbb{R} \times G^{-}(\mathbb{R}, \mathbb{R}) \rightarrow \mathbb{R}$ is given by

$$
\tilde{f}(t, \varphi)=\left\{\begin{array}{lll}
F(t, \varphi), & t \neq t_{k}, & k \in \mathbb{Z}, \\
b_{k}, & t=t_{k}, & k \in \mathbb{Z}
\end{array}\right.
$$

and $\tilde{u}$ is given by (4.6).

Since $x_{\lambda}(0)=\lambda x_{\lambda}(0)$ and $x_{\lambda}(T)=x_{\lambda}(0)$, it follows by (4.11) that

$$
\int_{0}^{T} \tilde{f}\left(t,\left(x_{\lambda}\right)_{t}\right) d \tilde{u}(t)=0 .
$$

Then,

$$
\int_{0}^{T} a(t) h\left(x_{\lambda}(t)\right) d t+\int_{0}^{T}\left(\int_{t-r}^{t} B(t, s) g\left(x_{\lambda}(s)\right) d s\right) d t+\int_{0}^{T} p(t) d t+\sum_{0<t_{k}<T} b_{k}=0 .
$$

Consequently,

$$
\begin{aligned}
& \left|\int_{0}^{T} a(t) h\left(x_{\lambda}(t)\right) d t\right| \leq \\
& \leq\left|\int_{0}^{T}\left(\int_{t-r}^{t} B(t, s) g\left(x_{\lambda}(s)\right) d s\right) d t\right|+\left|\int_{0}^{T} p(t) d t\right|+\sum_{0<t_{k}<T}\left|b_{k}\right| \\
& \leq \int_{0}^{T}\left(\int_{t-r}^{t}\left|B(t, s) g\left(x_{\lambda}(s)\right)\right| d s\right) d t+\int_{0}^{T} \tilde{P}(t) d t+q M \\
& \leq C \int_{0}^{T}\left(\int_{t-r}^{t} L_{g}(s) d s\right) d t+\int_{0}^{T} \tilde{P}(t) d t+q M:=\eta .
\end{aligned}
$$

Note that $0<\eta<\infty$ once conditions d) and f) hold. Moreover, since $a, h$ and $\tilde{u}$ are leftcontinuous functions, there exists $0<\delta<t_{0}, \delta=\delta(a, h, \tilde{u})$, such that $a, h$ and $\tilde{u}$ are continuous on $(0, \delta]$, where $t_{0}>0$ is the first impulsive moment. As described in Lemma 3.1.1, we conclude that $x_{\lambda}$ is continuous on $(0, \delta]$. Fix $t_{\delta} \in(0, \delta)$. As $t \mapsto a(t) h\left(x_{\lambda}(t)\right)$ is continuous on $\left[t_{\delta}, \delta\right]$, it follows from the Mean Value Theorem for integrals (see Theorem 2.4.23), that there exists $\xi \in\left[t_{\delta}, \delta\right]$ such that

$$
a(\xi) h\left(x_{\lambda}(\xi)\right)=\frac{1}{\delta-t_{\delta}} \int_{t_{\delta}}^{\delta} a(t) h\left(x_{\lambda}(t)\right) d t \leq \frac{\eta}{\delta-t_{\delta}} .
$$

Given $J>0$, it follows by e), the existence of $A>0$ such that, if $\left|x_{\lambda}(\xi)\right| \geq A$ then $h\left(x_{\lambda}(\xi)\right) \geq J\left|x_{\lambda}(\xi)\right|$. Consequently, using the fact that $a(\xi) \geq a_{0}$ and (4.13), we obtain

$$
a_{0} J\left|x_{\lambda}(\xi)\right| \leq a(\xi) h\left(x_{\lambda}(\xi)\right) \leq \frac{\eta}{\delta-t_{\delta}}
$$

provided $\left|x_{\lambda}(\xi)\right| \geq A$. Thus, $\left|x_{\lambda}(\xi)\right| \leq \max \left\{A, \frac{\eta}{a_{0} J\left(\delta-t_{\delta}\right)}\right\}:=v$.

Using f) and the fact that $\xi \in[0, T]$, we obtain for all $t \in[0, T]$, 


$$
\begin{aligned}
\left|x_{\lambda}(t)\right| & \leq\left|x_{\lambda}(\xi)\right|+\left|\int_{\xi}^{t} a(s) h\left(x_{\lambda}(s)\right) d s\right|+\left|\int_{\xi}^{t}\left(\int_{s-r}^{s} B(s, w) g\left(x_{\lambda}(w)\right) d w\right) d s\right| \\
& +\left|\int_{\xi}^{t} p(s) d s\right|+\sum_{\xi \leq t_{k}<t}\left|b_{k}\right| \\
& \leq v+\int_{0}^{T} a(s) h\left(x_{\lambda}(s)\right) d s+\int_{0}^{T}\left(\int_{s-r}^{s} B(s, w) g\left(x_{\lambda}(w)\right) d w\right) d s \\
& +\int_{0}^{T} \tilde{P}(s) d s+q M \\
& \quad \stackrel{(4.12)}{\leq} v+\left|\int_{0}^{T} p(s) d s\right|+\sum_{0<t_{k}<T}\left|b_{k}\right|+\int_{0}^{T} \tilde{P}(s) d s+q M \leq \beta
\end{aligned}
$$

where $\beta=v+2 \int_{0}^{T} \tilde{P}(s) d s+2 q M$.

Therefore, by Theorem 4.1.5, the impulsive nonlinear Volterra integrodifferential equation with delay (4.9) admits at least one $T$-periodic solution. 

CHAPTER

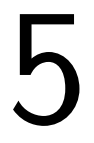

\section{NEUTRAL FUNCTIONAL DIFFERENTIAL EQUATIONS}

The aim of this chapter is to provide sufficient conditions to obtain periodic solutions for neutral functional differential equations (NFDEs for short) of type

$$
\frac{d}{d t}\left[x(t)-A\left(t, x_{t}\right)\right]=f\left(t, x_{t}\right)
$$

almost everywhere in $\mathbb{R}$, where $A, f: \mathbb{R} \times C\left(\mathbb{R}, \mathbb{R}^{n}\right) \rightarrow \mathbb{R}^{n}$ are $T$-periodic functions with respect to the first variable and $T>0$.

\subsection{Existence of periodic solutions}

In order to guarantee the existence of periodic solutions for the NFDE (5.1), we will apply the fixed-point Theorem 2.2.8 established by D. O'regan in (O'REGAN, 1996).

The next definition concerns the concept of a solution of the NFDE (5.1).

Definition 5.1.1. A function $x: \mathbb{R} \rightarrow \mathbb{R}^{n}$ is said to be a solution of the NFDE (5.1) if $x \in C\left(\mathbb{R}, \mathbb{R}^{n}\right)$ and $x$ satisfies (5.1) almost everywhere in $\mathbb{R}$.

Next, we present the general conditions that we shall consider in this section.

(J1) $A, f: \mathbb{R} \times C\left(\mathbb{R}, \mathbb{R}^{n}\right) \rightarrow \mathbb{R}^{n}$ are $T$-periodic functions with respect to the first variable;

Denote by $\Lambda_{A}^{I}$ the set of all discontinuity points of the map $I \ni t \mapsto A(t, \cdot)$ on the interval $I \subset \mathbb{R}$. Also, let $\left|\Lambda_{A}^{I}\right|$ be the cardinality of the set $\Lambda_{A}^{I}$.

(J2) the mapping $t \mapsto A(t, \cdot)$ is left continuous on $\mathbb{R}$ with $\left|\Lambda_{A}^{[0, T)}\right|<\infty$ and $t \mapsto A\left(t, x_{t}\right)$ is differentiable almost everywhere on $\mathbb{R}$ whenever $x \in C_{T}\left(\mathbb{R}, \mathbb{R}^{n}\right)$ is differentiable almost 
everywhere on $\mathbb{R}$. The mapping $t \mapsto f\left(t, x_{t}\right)$ is locally Perron integrable over $\mathbb{R}$ whenever $x \in C_{T}\left(\mathbb{R}, \mathbb{R}^{n}\right)$

(J3) given $\mu>0$, there are $\omega \in\left(0, \frac{1}{4 m+2}\right), m=\left|\Lambda_{A}^{[0, T)}\right|$, and a nondecreasing continuous function $\gamma_{\mu}: \mathbb{R}_{+} \rightarrow \mathbb{R}_{+}$satisfying $0 \leq \gamma_{\mu}(z) \leq \omega z$ for all $z \in \mathbb{R}_{+}$, such that

$$
\left\|A\left(t, x_{t}\right)-A\left(t, y_{t}\right)\right\| \leq \gamma_{\mu}\left(|x-y|_{T}\right)
$$

for all $t \in[0, T]$ and for all $x, y \in C_{T}\left(\mathbb{R}, \mathbb{R}^{n}\right)$ with $|x|_{T} \leq \mu$ and $|y|_{T} \leq \mu$;

(J4) given $\mu>0$, there is a non-negative locally Perron integrable function $F_{\mu}: \mathbb{R} \rightarrow \mathbb{R}_{+}$such that, for all $t_{1}, t_{2} \in[0, T], t_{1} \leq t_{2}$,

$$
\left\|\int_{t_{1}}^{t_{2}} f\left(t, x_{t}\right) d t\right\| \leq \int_{t_{1}}^{t_{2}} F_{\mu}(t) d t
$$

for every $x \in C_{T}\left(\mathbb{R}, \mathbb{R}^{n}\right)$ such that $|x|_{T} \leq \mu$;

(J5) given $\mu>0$, there is a non-negative locally Perron integrable function $L_{\mu}: \mathbb{R} \rightarrow \mathbb{R}_{+}$such that, for all $t_{1}, t_{2} \in[0, T], t_{1} \leq t_{2}$,

$$
\left\|\int_{t_{1}}^{t_{2}}\left[f\left(t, x_{t}\right)-f\left(t, y_{t}\right)\right] d t\right\| \leq \int_{t_{1}}^{t_{2}} L_{\mu}(t)|x-y|_{T} d t
$$

whenever $x, y \in C_{T}\left(\mathbb{R}, \mathbb{R}^{n}\right)$ is such that $|x|_{T} \leq \mu$ and $|y|_{T} \leq \mu$.

Let $\beta>0$. Now, let us define the set

$$
X_{\beta}=\left\{x \in C_{T}\left(\mathbb{R}, \mathbb{R}^{n}\right):|x|_{T} \leq \beta\right\} .
$$

Note that $X_{\beta}$ is a nonempty closed subset of the Banach space $\left(G_{T}^{-}\left(\mathbb{R}, \mathbb{R}^{n}\right),|\cdot|_{T}\right)$. In addition, we shall assume that $\Lambda_{A}^{[0, T)}=\left\{\lambda_{0}, \lambda_{1}, \ldots, \lambda_{m-1}\right\}$. If $\Lambda_{A}^{[0, T)}=\emptyset$ then the results are obtained analogously.

Remark 5.1.2. Since $A$ is $T$-periodic with respect to the first variable and

$$
\Lambda_{A}^{[0, T)}=\left\{\lambda_{0}, \lambda_{1}, \ldots, \lambda_{m-1}\right\},
$$

the mapping $t \mapsto A(t, \cdot)$ is discontinuous at the points $\ldots<\lambda_{-1}<\lambda_{0}<\lambda_{1}<\ldots$, where

$$
\lambda_{m+j}=\lambda_{j}+T, \text { for all } j \in \mathbb{Z} .
$$

Hence, for each $k \in \mathbb{Z}$, we have

$$
\Lambda_{A}^{[k T,(k+1) T)}=\left\{\lambda_{0}+k T, \lambda_{1}+k T, \ldots, \lambda_{m-1}+k T\right\}=\left\{\lambda_{k m}, \lambda_{k m+1}, \ldots, \lambda_{k m+m-1}\right\} .
$$

Moreover, if $\lambda_{j} \in \Lambda_{A}^{[k T,(k+1) T)}$ then there is $i \in\{0,1, \ldots, m-1\}$ such that $\lambda_{j}=\lambda_{i}+k T$, where $\lambda_{i} \in \Lambda_{A}^{[0, T)}$. Thus, by periodicity of $A$ we have

$$
A\left(\lambda_{j}, x_{\lambda_{j}}\right)-A\left(\lambda_{j}^{+}, x_{\lambda_{j}}\right)=A\left(\lambda_{i}, x_{\lambda_{i}}\right)-A\left(\lambda_{i}^{+}, x_{\lambda_{i}}\right),
$$

whenever $x$ is $T$-periodic. 
As presented in Theorem 2.2.8, we need to construct two maps: a nonlinear contraction and a completely continuous map. In this way, let us define the map $H: X_{\beta} \rightarrow G_{T}^{-}\left(\mathbb{R}, \mathbb{R}^{n}\right)$ by

$$
H(x)(t)=\Phi(x)(t)+\Psi(x)(t),
$$

where $\Phi, \Psi: X_{\beta} \rightarrow G_{T}^{-}\left(\mathbb{R}, \mathbb{R}^{n}\right)$ are given by

$$
\Phi(x)(t)=A\left(t, x_{t}\right)+\sum_{k T<\lambda_{j}<t}\left[A\left(\lambda_{j}, x_{\lambda_{j}}\right)-A\left(\lambda_{j}^{+}, x_{\lambda_{j}}\right)\right], \quad t \in(k T,(k+1) T], k \in \mathbb{Z},
$$

and

$$
\Psi(x)(t)=\int_{k T}^{t} f\left(s, x_{s}\right) d s, \quad t \in(k T,(k+1) T], k \in \mathbb{Z} .
$$

We will prove that $\Phi$ is a nonlinear contraction and $\Psi$ is a completely continuous map.

Lemma 5.1.3. Assume that conditions ( $\mathrm{J} 1)$ and $(\mathrm{J} 2)$ hold. Then, the function $H$ is well-defined.

Proof. Let $x \in X_{\beta}$. By condition (J2) and Theorem 2.4.13, we get $H(x) \in G^{-}\left(\mathbb{R}, \mathbb{R}^{n}\right)$. Now, let $t \in \mathbb{R}$ and $k \in \mathbb{Z}$ be such that $k T<t \leq(k+1) T$. Thus, by Remark 5.1.2

$$
\begin{aligned}
\Phi(x)(t+T) & =A\left(t+T, x_{t+T}\right)+\sum_{(k+1) T<\lambda_{j}<t+T}\left[A\left(\lambda_{j}, x_{\lambda_{j}}\right)-A\left(\lambda_{j}^{+}, x_{\lambda_{j}}\right)\right] \\
& =A\left(t, x_{t}\right)+\sum_{k T<\lambda_{j}-T<t}\left[A\left(\lambda_{j}-T, x_{\lambda_{j}-T}\right)-A\left(\left(\lambda_{j}-T\right)^{+}, x_{\lambda_{j}-T}\right)\right] \\
& =A\left(t, x_{t}\right)+\sum_{k T<\lambda_{i}<t}\left[A\left(\lambda_{i}, x_{\lambda_{i}}\right)-A\left(\lambda_{i}^{+}, x_{\lambda_{i}}\right)\right] \\
& =\Phi(x)(t) .
\end{aligned}
$$

On the other hand, since $f: \mathbb{R} \times C\left(\mathbb{R}, \mathbb{R}^{n}\right) \rightarrow \mathbb{R}^{n}$ is $T$-periodic with respect to the first variable (see condition (J1)), we may use Theorem 2.4.9 to obtain

$$
\Psi(x)(t+T)=\int_{(k+1) T}^{t+T} f\left(s, x_{s}\right) d s=\int_{k T}^{t} f\left(s+T, x_{s+T}\right) d s=\int_{k T}^{t} f\left(s, x_{s}\right) d s=\Psi(x)(t) .
$$

Hence, $H \in G_{T}^{-}\left(\mathbb{R}, \mathbb{R}^{n}\right)$ and $H$ is well-defined.

In what follows, we exhibit some auxiliary results.

Lemma 5.1.4. Assume that conditions (J1)-(J4) hold. Then $H\left(X_{\beta}\right)$ is bounded in $G_{T}^{-}\left(\mathbb{R}, \mathbb{R}^{n}\right)$.

Proof. Let $x \in X_{\beta}$ be arbitrary. It follows from (5.2), the periodicity of $\Phi(x)$ and condition (J3) that

$$
\begin{aligned}
|\Phi(x)|_{T} & =\sup _{t \in(0, T]}\|\Phi(x)(t)\| \\
& \leq \sup _{t \in(0, T]}\left\|A\left(t, x_{t}\right)\right\|+\sum_{0<\lambda_{j}<T}\left\|A\left(\lambda_{j}, x_{\lambda_{j}}\right)-A\left(\lambda_{j}^{+}, x_{\lambda_{j}}\right)\right\| \\
& \leq(2 m+1) \gamma_{\beta}\left(|x|_{T}\right)+c \\
& \leq(2 m+1) \omega \beta+c,
\end{aligned}
$$


with $c=\sup _{t \in[0, T]}\|A(t, 0)\|+\sum_{0<\lambda_{j}<T}\left(\left\|A\left(\lambda_{j}, 0\right)\right\|+\left\|A\left(\lambda_{j}^{+}, 0\right)\right\|\right)<\infty$ as $t \mapsto A(t, 0)$ is regulated on $\mathbb{R}$.

On the other hand, since $\Psi(x)(T)=\Psi(x)(0)$, it follows by (5.3) and condition (J4) that

$$
|\Psi(x)|_{T}=\sup _{t \in(0, T]}\|\Psi(x)(t)\| \leq \sup _{t \in(0, T]}\left\|\int_{0}^{t} f\left(s, x_{s}\right) d s\right\| \leq \int_{0}^{T} F_{\beta}(s) d s .
$$

Therefore, $H\left(X_{\beta}\right)$ is bounded in $G_{T}^{-}\left(\mathbb{R}, \mathbb{R}^{n}\right)$.

Lemma 5.1.5. Suppose (J4) holds. Then $\Psi\left(X_{\beta}\right)$ is relatively compact.

Proof. Let $\left\{x_{n}\right\}_{n \in \mathbb{N}}$ be a sequence in $X_{\beta}$. Note that (5.4) implies that the sequence $\left\{\Psi\left(x_{n}\right)\right\}_{n \in \mathbb{N}}$ is uniformly bounded in $[0, T]$. Moreover, using condition (J4), for all $t_{1}, t_{2} \in(0, T], t_{1} \leq t_{2}$, we have

$$
\begin{aligned}
\left\|\Psi\left(x_{n}\right)\left(t_{2}\right)-\Psi\left(x_{n}\right)\left(t_{1}\right)\right\| & =\left\|\int_{t_{1}}^{t_{2}} f\left(s, x_{s}\right) d s\right\| \\
& \leq \int_{t_{1}}^{t_{2}} F_{\beta}(s) d s \\
& \leq\left|h\left(t_{2}\right)-h\left(t_{1}\right)\right|,
\end{aligned}
$$

where $h(t)=\int_{0}^{t} F_{\beta}(s) d s$ for $t \in[0, T]$. Hence, by Theorems 2.4.13 and 2.3.4, $\left\{\Psi\left(x_{n}\right)\right\}_{n \in \mathbb{N}}$ is equiregulated on $[0, T]$. Therefore, by Theorem 2.3.7, up to a subsequence, we may assume that the sequence $\left\{\left.\Psi\left(x_{n}\right)\right|_{[0, T]}\right\}_{n \in \mathbb{N}}$ converges uniformly on $[0, T]$ to a function $y_{0}:[0, T] \rightarrow \mathbb{R}^{n}$. Note that $y_{0}(0)=y_{0}(T)$. Moreover, the function $y: \mathbb{R} \rightarrow \mathbb{R}^{n}$ given by

$$
y(t)=y_{0}(t-k T), \quad k T<t \leq(k+1) T, k \in \mathbb{Z},
$$

is a limit of $\left\{\Psi\left(x_{n}\right)\right\}_{n \in \mathbb{N}}$, which completes the proof.

Lemma 5.1.6. Assume that condition (J5) holds. Then $\Psi$ is a continuous mapping on $X_{\beta}$.

Proof. Let $x, y \in X_{\beta}$. Using the $T$-periodicity of $\Psi(x)$ and $\Psi(y)$, and condition (J5), we have

$$
|\Psi(x)-\Psi(y)|_{T} \leq \sup _{t \in(0, T]}\left\|\int_{0}^{t}\left[f\left(s, x_{s}\right)-f\left(s, y_{s}\right)\right] d s\right\| \leq\left(\int_{0}^{T} L_{\beta}(s) d s\right)|x-y|_{T},
$$

which shows the continuity of $\Psi$.

Lemma 5.1.7. Assume that $(\mathrm{J} 3)$ holds. Then $\Phi$ is a nonlinear contraction.

Proof. Let $x, y \in X_{\beta}$. Using condition (J3) and the $T$-periodicity of $\Phi(x)$ and $\Phi(y)$, we obtain

$$
\begin{aligned}
|\Phi(x)-\Phi(y)|_{T} & \leq \sup _{t \in(0, T]}\left\|A\left(t, x_{t}\right)-A\left(t, y_{t}\right)\right\|+\sum_{0<\lambda_{j}<T}\left\|A\left(\lambda_{j}, x_{\lambda_{j}}\right)-A\left(\lambda_{j}, y_{\lambda_{j}}\right)\right\| \\
& +\sum_{0<\lambda_{j}<T}\left\|A\left(\lambda_{j}^{+}, x_{\lambda_{j}}\right)-A\left(\lambda_{j}^{+}, y_{\lambda_{j}}\right)\right\| \\
& \leq(1+2 m) \gamma_{\beta}\left(|x-y|_{T}\right)=\Lambda\left(|x-y|_{T}\right),
\end{aligned}
$$


where $\Lambda(z)=(1+2 m) \gamma_{\beta}(z) \leq(1+2 m) \omega z<z$ for all $z \in \mathbb{R}_{+}$. Hence, $\Phi$ is a nonlinear contraction.

Lemma 5.1.8. If $x \in X_{\beta}$ is such that $x=H(x)$, then

$$
\int_{t}^{t+T} f\left(s, x_{s}\right) d s+\sum_{t<\lambda_{j} \leq t+T}\left[A\left(\lambda_{j}, x_{\lambda_{j}}\right)-A\left(\lambda_{j}^{+}, x_{\lambda_{j}}\right)\right]=0
$$

for all $t \in \mathbb{R}$.

Proof. Let $x \in X_{\beta}$ be such that $x=H(x)$ and $t \in(0, T]$. By (5.2)-(5.3) we may write

$$
x(t)=H(x)(t)=A\left(t, x_{t}\right)+\sum_{0<\lambda_{j}<t}\left[A\left(\lambda_{j}, x_{\lambda_{j}}\right)-A\left(\lambda_{j}^{+}, x_{\lambda_{j}}\right)\right]+\int_{0}^{t} f\left(s, x_{s}\right) d s .
$$

Since $\Lambda_{A}^{[0, T)}=\left\{\lambda_{0}, \lambda_{1}, \ldots, \lambda_{m-1}\right\}$, there exists $\delta>0$ such that

$$
x(t)-A\left(t, x_{t}\right)=\int_{0}^{t} f\left(s, x_{s}\right) d s \quad \text { for } \quad 0<t<\delta .
$$

We may choose $\delta=\lambda_{0}$ if $\lambda_{0}>0$ or $\delta=\lambda_{1}$ if $\lambda_{0}=0$. Using Theorem 2.4.13 and the fact that $x$ is continuous and $t \mapsto A\left(t, x_{t}\right)$ is left-continuous, we get

$$
0=\lim _{t \rightarrow 0^{+}}\left[x(t)-A\left(t, x_{t}\right)\right]=x(0)-A\left(0^{+}, x_{0}\right) .
$$

Now, since $x(T)=x(0), A\left(T, x_{T}\right)=A\left(0, x_{0}\right)$ and (5.6) holds, we obtain from (5.5), when $t \rightarrow T^{-}$, the following equality

$$
A\left(0^{+}, x_{0}\right)-A\left(0, x_{0}\right)=x(T)-A\left(T, x_{T}\right)=\sum_{0<\lambda_{j}<T}\left[A\left(\lambda_{j}, x_{\lambda_{j}}\right)-A\left(\lambda_{j}^{+}, x_{\lambda_{j}}\right)\right]+\int_{0}^{T} f\left(s, x_{s}\right) d s .
$$

Since $\lambda_{0} \geq 0$, we conclude that

$$
\sum_{0 \leq \lambda_{j}<T}\left[A\left(\lambda_{j}, x_{\lambda_{j}}\right)-A\left(\lambda_{j}^{+}, x_{\lambda_{j}}\right)\right]+\int_{0}^{T} f\left(s, x_{s}\right) d s=0 .
$$

But, using the $T$-periodicity, we get

$$
\sum_{0<\lambda_{j} \leq T}\left[A\left(\lambda_{j}, x_{\lambda_{j}}\right)-A\left(\lambda_{j}^{+}, x_{\lambda_{j}}\right)\right]+\int_{0}^{T} f\left(s, x_{s}\right) d s=0 .
$$

Now, let $t \in \mathbb{R}$ and $k \in \mathbb{Z}$ be such that $k T<t \leq(k+1) T$. Using Theorem 2.4.9 and the periodicity of $f$ and $x$, we get

$$
\begin{aligned}
\int_{t}^{t+T} f\left(s, x_{s}\right) d s & =\int_{t}^{k T} f\left(s, x_{s}\right) d s+\int_{k T}^{(k+1) T} f\left(s, x_{s}\right) d s+\int_{(k+1) T}^{t+T} f\left(s, x_{s}\right) d s \\
& =\int_{t}^{k T} f\left(s, x_{s}\right) d s+\int_{0}^{T} f\left(s, x_{s}\right) d s+\int_{k T}^{t} f\left(s, x_{s}\right) d s \\
& =\int_{0}^{T} f\left(s, x_{s}\right) d s .
\end{aligned}
$$


By Remark 5.1.2,

$$
\begin{aligned}
\sum_{t<\lambda_{j} \leq t+T} & {\left[A\left(\lambda_{j}, x_{\lambda_{j}}\right)-A\left(\lambda_{j}^{+}, x_{\lambda_{j}}\right)\right]=} \\
= & \sum_{t<\lambda_{j} \leq(k+1) T}\left[A\left(\lambda_{j}, x_{\lambda_{j}}\right)-A\left(\lambda_{j}^{+}, x_{\lambda_{j}}\right)\right]+\sum_{(k+1) T<\lambda_{j} \leq(k+2) T}\left[A\left(\lambda_{j}, x_{\lambda_{j}}\right)-A\left(\lambda_{j}^{+}, x_{\lambda_{j}}\right)\right] \\
& -\sum_{t+T<\lambda_{j} \leq(k+2) T}\left[A\left(\lambda_{j}, x_{\lambda_{j}}\right)-A\left(\lambda_{j}^{+}, x_{\lambda_{j}}\right)\right] \\
= & \sum_{t<\lambda_{j} \leq(k+1) T}\left[A\left(\lambda_{j}, x_{\lambda_{j}}\right)-A\left(\lambda_{j}^{+}, x_{\lambda_{j}}\right)\right]+\sum_{0<\lambda_{i} \leq T}\left[A\left(\lambda_{i}, x_{\lambda_{i}}\right)-A\left(\lambda_{i}^{+}, x_{\lambda_{i}}\right)\right] \\
& -\sum_{t<\lambda_{j}-T \leq(k+1) T}\left[A\left(\lambda_{j}-T, x_{\lambda_{j}-T}\right)-A\left(\left(\lambda_{j}-T\right)^{+}, x_{\lambda_{j}-T}\right)\right] \\
= & \sum_{0<\lambda_{i} \leq T}\left[A\left(\lambda_{i}, x_{\lambda_{i}}\right)-A\left(\lambda_{i}^{+}, x_{\lambda_{i}}\right)\right] .
\end{aligned}
$$

Hence, by (5.7), (5.8) and (5.9), it follows that

$$
\sum_{t<\lambda_{j} \leq t+T}\left[A\left(\lambda_{j}, x_{\lambda_{j}}\right)-A\left(\lambda_{j}^{+}, x_{\lambda_{j}}\right)\right]+\int_{t}^{t+T} f\left(s, x_{s}\right) d s=0 \quad \text { for all } \quad t \in \mathbb{R} .
$$

Now, we may state the theorem on the existence of periodic solutions for the NFDE $(5.1)$.

Theorem 5.1.9. Assume that conditions (J1)-(J5) hold. If $\beta>0$ is such that

$$
\sup _{t \in[0, T]}\|A(t, 0)\|+\sum_{0<\lambda_{j}<T}\left(\left\|A\left(\lambda_{j}, 0\right)\right\|+\left\|A\left(\lambda_{j}^{+}, 0\right)\right\|\right)+\int_{0}^{T} F_{\beta}(s) d s \leq \frac{\beta}{2},
$$

then the NFDE (5.1) admits a $T$-periodic solution $x \in X_{\beta}$ which satisfies the following integral equation

$$
x(t)=\left\{\begin{array}{l}
A\left(t, x_{t}\right)+\sum_{0<\lambda_{j}<t}\left[A\left(\lambda_{j}, x_{\lambda_{j}}\right)-A\left(\lambda_{j}^{+}, x_{\lambda_{j}}\right)\right]+\int_{0}^{t} f\left(s, x_{s}\right) d s, \quad t>0, \\
A\left(t, x_{t}\right)-\sum_{t \leq \lambda_{j} \leq 0}\left[A\left(\lambda_{j}, x_{\lambda_{j}}\right)-A\left(\lambda_{j}^{+}, x_{\lambda_{j}}\right)\right]+\int_{0}^{t} f\left(s, x_{s}\right) d s, \quad t \leq 0 .
\end{array}\right.
$$

Proof. Due to condition (J3), we have the following estimate

$$
\left\|A\left(t, x_{t}\right)-A\left(t, y_{t}\right)\right\|<\frac{1}{4 m+2}|x-y|_{T}
$$

for all $t \in[0, T]$ and for all $x, y \in X_{\beta}$. Now, by Lemmas 5.1.4, 5.1.5, 5.1.6 and 5.1.7, $H\left(X_{\beta}\right)$ is bounded, $\Psi$ is completely continuous and $\Phi$ is a nonlinear contraction.

We claim that $H$ has a fixed point in $X_{\beta}$. Indeed, suppose to the contrary, that there are $x \in \partial X_{\beta}=\left\{x \in C_{T}\left(\mathbb{R}, \mathbb{R}^{n}\right):|x|_{T}=\beta\right\}$ and $\lambda \in(0,1)$ such that $x=\lambda H(x)$. Let $t^{*} \in[0, T]$ 
be such that $\left\|x\left(t^{*}\right)\right\|=\sup _{t \in[0, T]}\|x(t)\|$. Since $x(0)=x(T)$, we may assume without loss of generality that $t^{*} \in(0, T]$. Thus, by condition (J4), relations (5.10) and (5.11), we get

$$
\begin{aligned}
\beta & =\left\|x\left(t^{*}\right)\right\| \leq\left\|A\left(t^{*}, x_{t^{*}}\right)-A\left(t^{*}, 0\right)\right\|+\left\|A\left(t^{*}, 0\right)\right\| \\
& +\sum_{0<\lambda_{j}<t^{*}}\left\|A\left(\lambda_{j}, x_{\lambda_{j}}\right)-A\left(\lambda_{j}, 0\right)\right\|+\sum_{0<\lambda_{j}<t^{*}}\left\|A\left(\lambda_{j}^{+}, x_{\lambda_{j}}\right)-A\left(\lambda_{j}^{+}, 0\right)\right\| \\
& +\sum_{0<\lambda_{j}<t^{*}}\left(\left\|A\left(\lambda_{j}, 0\right)\right\|+\left\|A\left(\lambda_{j}^{+}, 0\right)\right\|\right)+\left\|\int_{0}^{t^{*}} f\left(s, x_{s}\right) d s\right\| \\
& <(2 m+1) \frac{1}{4 m+2}|x|_{T}+\left\|A\left(t^{*}, 0\right)\right\|+\sum_{0<\lambda_{j}<t^{*}}\left(\left\|A\left(\lambda_{j}, 0\right)\right\|+\left\|A\left(\lambda_{j}^{+}, 0\right)\right\|\right)+\int_{0}^{t^{*}} F_{\beta}(s) d s \\
& \leq \frac{\beta}{2}+\frac{\beta}{2}=\beta,
\end{aligned}
$$

which is a contradiction. Therefore, by Theorem 2.2.8, there is $x^{*} \in X_{\beta}$ such that $x^{*}=H\left(x^{*}\right)$, that is,

$$
x^{*}(t)-A\left(t, x_{t}^{*}\right)=\sum_{k T<\lambda_{j}<t}\left[A\left(\lambda_{j}, x_{\lambda_{j}}^{*}\right)-A\left(\lambda_{j}^{+}, x_{\lambda_{j}}^{*}\right)\right]+\int_{k T}^{t} f\left(s, x_{s}^{*}\right) d s,
$$

for all $t \in(k T,(k+1) T], k \in \mathbb{Z}$. Using Theorem 2.4.19, we conclude that $x^{*}(t)-A\left(t, x_{t}^{*}\right)$ is differentiable almost everywhere on $\mathbb{R}$ and

$$
\begin{aligned}
& \frac{d}{d t}\left[x^{*}(t)-A\left(t, x_{t}^{*}\right)\right]= \\
& =\frac{d}{d t}\left(\sum_{k T<\lambda_{j}<t}\left[A\left(\lambda_{j}, x_{\lambda_{j}}^{*}\right)-A\left(\lambda_{j}^{+}, x_{\lambda_{j}}^{*}\right)\right]\right)+\frac{d}{d t}\left(\int_{k T}^{t} f\left(s, x_{s}^{*}\right) d s\right) \\
& =f\left(t, x_{t}^{*}\right) .
\end{aligned}
$$

Therefore, $x^{*}$ satisfies the equation (5.1) almost everywhere in $\mathbb{R}$. Hence, $x^{*}$ is a $T$-periodic solution of the NFDE (5.1).

Let $t>0$ and $k \in \mathbb{Z}, k \geq 0$, be such that $t \in(k T,(k+1) T]$. By Lemma 5.1.8, we have

$$
\sum_{i=0}^{k-1}\left(\int_{i T}^{(i+1) T} f\left(s, x_{s}^{*}\right) d s+\sum_{i T<\lambda_{j} \leq(i+1) T}\left[A\left(\lambda_{j}, x_{\lambda_{j}}^{*}\right)-A\left(\lambda_{j}^{+}, x_{\lambda_{j}}^{*}\right)\right]\right)=0,
$$

consequently,

$$
\begin{aligned}
& \sum_{0<\lambda_{j}<t}\left[A\left(\lambda_{j}, x_{\lambda_{j}}^{*}-A\left(\lambda_{j}^{+}, x_{\lambda_{j}}^{*}\right)\right]\right)+\int_{0}^{t} f\left(s, x_{s}^{*}\right) d s= \\
= & \sum_{i=0}^{k-1}\left(\sum_{i T<\lambda_{j} \leq(i+1) T}\left[A\left(\lambda_{j}, x_{\lambda_{j}}^{*}\right)-A\left(\lambda_{j}^{+}, x_{\lambda_{j}}^{*}\right)\right]+\sum_{k T<\lambda_{j}<t}\left[A\left(\lambda_{j}, x_{\lambda_{j}}^{*}\right)-A\left(\lambda_{j}^{+}, x_{\lambda_{j}}^{*}\right)\right]\right. \\
+ & \sum_{i=0}^{k-1}\left(\int_{i T}^{(i+1) T} f\left(s, x_{s}^{*}\right) d s\right)+\int_{k T}^{t} f\left(s, x_{s}^{*}\right) d s \\
= & \sum_{k T<\lambda_{j}<t}\left[A\left(\lambda_{j}, x_{\lambda_{j}}^{*}\right)-A\left(\lambda_{j}^{+}, x_{\lambda_{j}}^{*}\right)\right]+\int_{k T}^{t} f\left(s, x_{s}^{*}\right) d s .
\end{aligned}
$$


Therefore,

$$
\begin{aligned}
x^{*}(t) & =A\left(t, x_{t}^{*}\right)+\sum_{k T<\lambda_{j}<t}\left[A\left(\lambda_{j}, x_{\lambda_{j}}^{*}\right)-A\left(\lambda_{j}^{+}, x_{\lambda_{j}}^{*}\right)\right]+\int_{k T}^{t} f\left(s, x_{s}^{*}\right) d s \\
& =A\left(t, x_{t}^{*}\right)+\sum_{0<\lambda_{j}<t}\left[A\left(\lambda_{j}, x_{\lambda_{j}}^{*}\right)-A\left(\lambda_{j}^{+}, x_{\lambda_{j}}^{*}\right)\right]+\int_{0}^{t} f\left(s, x_{s}^{*}\right) d s .
\end{aligned}
$$

Now, let $t \leq 0$ and $p \in \mathbb{Z}, p \leq-1$, be such that $t \in(p T,(p+1) T]$. Using Lemma 5.1.8 we get

$$
\sum_{i=0}^{-p-1}\left(\int_{(p+i) T}^{(p+1+i) T} f\left(s, x_{s}^{*}\right) d s+\sum_{(p+i) T<\lambda_{j} \leq(p+1+i) T}\left[A\left(\lambda_{j}, x_{\lambda_{j}}^{*}\right)-A\left(\lambda_{j}^{+}, x_{\lambda_{j}}^{*}\right)\right]\right)=0
$$

Hence, by (5.13)

$$
\begin{aligned}
& \sum_{p T<\lambda_{j}<t}\left[A\left(\lambda_{j}, x_{\lambda_{j}}^{*}\right)-A\left(\lambda_{j}^{+}, x_{\lambda_{j}}^{*}\right)\right]+\int_{p T}^{t} f\left(s, x_{s}^{*}\right) d s= \\
= & \sum_{i=0}^{-p-1}\left(\sum_{(p+i) T<\lambda_{j} \leq(p+1+i) T}\left[A\left(\lambda_{j}, x_{\lambda_{j}}^{*}\right)-A\left(\lambda_{j}^{+}, x_{\lambda_{j}}^{*}\right)\right]\right)-\sum_{t \leq \lambda_{j} \leq 0}\left[A\left(\lambda_{j}, x_{\lambda_{j}}^{*}\right)-A\left(\lambda_{j}^{+}, x_{\lambda_{j}}^{*}\right)\right] \\
+ & \sum_{i=0}^{-p-1}\left(\int_{(p+i) T}^{(p+1+i) T} f\left(s, x_{s}^{*}\right) d s\right)+\int_{0}^{t} f\left(s, x_{s}^{*}\right) d s \\
= & -\sum_{t \leq \lambda_{j} \leq 0}\left[A\left(\lambda_{j}, x_{\lambda_{j}}^{*}\right)-A\left(\lambda_{j}^{+}, x_{\lambda_{j}}^{*}\right)\right]+\int_{0}^{t} f\left(s, x_{s}^{*}\right) d s .
\end{aligned}
$$

Thus,

$$
x^{*}(t)=A\left(t, x_{t}^{*}\right)-\sum_{t \leq \lambda_{j} \leq 0}\left[A\left(\lambda_{j}, x_{\lambda_{j}}\right)-A\left(\lambda_{j}^{+}, x_{\lambda_{j}}\right)\right]+\int_{0}^{t} f\left(s, x_{s}^{*}\right) d s \quad \text { for all } \quad t \leq 0
$$

and the proof is complete.

The next result provides us sufficient conditions for uniqueness of periodic solutions of the NFDE (5.1).

Theorem 5.1.10. Under the conditions of Theorem 5.1.9, assume that

$$
\int_{0}^{T} L_{\beta}(t) d t<\frac{1}{2}
$$

Then the NFDE (5.1) has a unique $T$-periodic solution in $X_{\beta}$. 
Proof. By (J3), (J4) and (5.10), we have $H$ maps $X_{\beta}$ into $X_{\beta}$. Indeed, as we saw in the proof of Theorem 5.1.9, for all $t \in[0, T)$, we obtain

$$
\begin{aligned}
\|H(x)(t)\| & <(2 m+1) \frac{1}{4 m+2}|x|_{T}+\|A(t, 0)\| \\
& +\sum_{0<\lambda_{j}<t}\left(\left\|A\left(\lambda_{j}, 0\right)\right\|+\left\|A\left(\lambda_{j}^{+}, 0\right)\right\|\right)+\int_{0}^{t} F_{\beta}(s) d s \\
& \leq \frac{\beta}{2}+\frac{\beta}{2}=\beta,
\end{aligned}
$$

since $x \in X_{\beta}$. Hence, taking the supremum over all $t \in[0, T]$ we have $|H(x)|_{T} \leq \beta$ and then, $H(x) \in X_{\beta}$. Now, using conditions (J3) and (J5), we get

$$
\begin{aligned}
\|H(x)(t)-H(y)(t)\| & \leq(2 m+1) \omega|x-y|_{T}+\int_{0}^{t} L_{\beta}(t)|x-y|_{T} d t \\
& <\frac{1}{2}|x-y|_{T}+\int_{0}^{t} L_{\beta}(t)|x-y|_{T} d t,
\end{aligned}
$$

for all $x, y \in X_{\beta}$ and all $t \in(0, T]$. Consequently, by taking the supremum over all $t \in[0, T]$ on both side of the above inequality, we obtain

$$
|H(x)-H(y)|_{T} \leq\left(\frac{1}{2}+\int_{0}^{T} L_{\beta}(t) d t\right)|x-y|_{T},
$$

for all $x, y \in X_{\beta}$. The result follows by using the Banach Fixed Point Theorem taking into account condition (5.14).

Remark 5.1.11. If $\Lambda_{A}^{[0, T)}=\emptyset$ in condition (J2) then $m=0$ in condition (J3), that is, $\omega \in\left(0, \frac{1}{2}\right)$.

\subsection{Examples}

This section deals with some applications referring to the theory of the previous section, where functions $t \mapsto A(t, \cdot)$ and $f$ are inserted in the general context of regulated functions and integrable functions in the sense of Perron, respectively.

Example 5.2.1. Consider the neutral delay differential equation

$$
\frac{d}{d t}(x(t)-q(t) x(t-r))=a(t) x(t)+b(t) x(t-r)+p(t)
$$

almost everywhere in $\mathbb{R}$, under the following conditions:

(a) $r \in \mathbb{R}, T>0$ and $a, b \in G_{T}^{-}(\mathbb{R}, \mathbb{R})$;

(b) $q: \mathbb{R} \rightarrow \mathbb{R}$ is $T$-periodic, left-continuous and differentiable almost everywhere on $\mathbb{R}$; $|q|_{T} \leq q^{*}$ with $0<q^{*}<\frac{1}{4 m+2}$ where $m=\left|\Lambda_{q}^{[0, T)}\right|$

(c) for every $x \in C_{T}\left(\mathbb{R}, \mathbb{R}^{n}\right)$ the mappings $t \mapsto a(t) x(t)$ and $t \mapsto b(t) x(t-r)$ are locally Perron integrable over $\mathbb{R}$; 
(d) there are non-negative locally Perron integrable functions $M, Q: \mathbb{R} \rightarrow \mathbb{R}_{+}$such that, for all $t_{1}, t_{2} \in[0, T], t_{1} \leq t_{2}$,

$$
\begin{aligned}
& \left\|\int_{t_{1}}^{t_{2}} a(t)\left[z_{1}(t)-z_{2}(t)\right] d t\right\| \leq \int_{t_{1}}^{t_{2}} M(t)\left|z_{1}-z_{2}\right|_{T} d t, \quad \text { and } \\
& \left\|\int_{t_{1}}^{t_{2}} b(t)\left[z_{1}(t-r)-z_{2}(t-r)\right] d t\right\| \leq \int_{t_{1}}^{t_{2}} Q(t)\left|z_{1}-z_{2}\right|_{T} d t,
\end{aligned}
$$

whenever $z_{1}, z_{2} \in C_{T}\left(\mathbb{R}, \mathbb{R}^{n}\right)$;

(e) $p: \mathbb{R} \rightarrow \mathbb{R}^{n}$ is $T$-periodic and locally Perron integrable function over $\mathbb{R}$, and there is non-negative locally Perron integrable function $P: \mathbb{R} \rightarrow \mathbb{R}_{+}$such that, for all $t_{1}, t_{2} \in[0, T]$, $t_{1} \leq t_{2}$

$$
\left\|\int_{t_{1}}^{t_{2}} p(t) d t\right\| \leq \int_{t_{1}}^{t_{2}} P(t) d t
$$

(f) $\int_{0}^{T}[M(t)+Q(t)] d t \leq \gamma$, for some $\gamma \in\left(0, \frac{1}{2}\right)$.

Let $f, A: \mathbb{R} \times C\left(\mathbb{R}, \mathbb{R}^{n}\right) \rightarrow \mathbb{R}^{n}$ be defined by

$$
f(t, \phi)=a(t) \phi(0)+b(t) \phi(-r)+p(t) \quad \text { and } \quad A(t, \phi)=q(t) \phi(-r),
$$

for all $t \in \mathbb{R}$ and all $\phi \in C\left(\mathbb{R}, \mathbb{R}^{n}\right)$. Let us show that conditions (J1)-(J5) hold.

Conditions (J1)-(J2) follow immediately from the assumptions (a), (b) and (c).

In order to prove (J3), take $\mu>0$ arbitrarily and let $x, y \in C_{T}\left(\mathbb{R}, \mathbb{R}^{n}\right)$ be such that $|x|_{T} \leq \mu$ and $|y|_{T} \leq \mu$. Then, by item (b), for every $t \in[0, T]$, we have

$$
\left\|A\left(t, x_{t}\right)-A\left(t, y_{t}\right)\right\|=|q(t)|\|x(t-r)-y(t-r)\| \leq \gamma_{\mu}\left(|x-y|_{T}\right),
$$

where $\gamma_{\mu}(s)=q^{*} s$ for all $s \in[0, \infty)$.

To verify that condition (J4) is satisfied, consider $\mu>0$ arbitrary and let $x \in C_{T}\left(\mathbb{R}, \mathbb{R}^{n}\right)$ be such that $|x|_{T} \leq \mu$. By (d) and (e), we get, for all $t_{1}, t_{2} \in[0, T], t_{1} \leq t_{2}$,

$$
\begin{aligned}
& \left\|\int_{t_{1}}^{t_{2}} f\left(t, x_{t}\right) d t\right\|= \\
& =\left\|\int_{t_{1}}^{t_{2}}[a(t) x(t)+b(t) x(t-r)+p(t)] d t\right\| \\
& \leq \int_{t_{1}}^{t_{2}} M(t)|x|_{T} d t+\int_{t_{1}}^{t_{2}} Q(t)|x|_{T} d t+\int_{t_{1}}^{t_{2}} P(t) d t \\
& \leq \int_{t_{1}}^{t_{2}}[(M(t)+Q(t)) \mu+P(t)] d t=\int_{t_{1}}^{t_{2}} F_{\mu}(t) d t,
\end{aligned}
$$

where $F_{\mu}(t)=[M(t)+Q(t)] \mu+P(t)$, for all $t \in \mathbb{R}$. 
Finally, we affirm that condition (J5) is satisfied. Indeed, let $x, y \in C_{T}\left(\mathbb{R}, \mathbb{R}^{n}\right)$ be such that $|x|_{T} \leq \mu$ and $|y|_{T} \leq \mu$. By items (d) and (e), we obtain

$$
\begin{aligned}
& \left\|\int_{t_{1}}^{t_{2}}\left[f\left(t, x_{t}\right)-f\left(t, y_{t}\right)\right] d t\right\| \leq \\
& \leq\left\|\int_{t_{1}}^{t_{2}} a(t)[x(t)-y(t)] d t\right\|+\left\|\int_{t_{1}}^{t_{2}} b(t)[x(t-r)-y(t-r)] d t\right\| \\
& \leq \int_{t_{1}}^{t_{2}}[M(t)+Q(t)]|x-y|_{T} d t,
\end{aligned}
$$

for all $t_{1}, t_{2} \in[0, T], t_{1} \leq t_{2}$. Hence, it is enough to take $L_{\mu}(t)=M(t)+Q(t)$, for $t \in \mathbb{R}$.

Since $q^{*}<\frac{1}{2}, A(t, 0)=0$ for every $t \in \mathbb{R}$, and condition (f) holds, we have

$$
\begin{gathered}
\sup _{t \in[0, T]}\|A(t, 0)\|+\sum_{0<\lambda_{j}<T}\left(\left\|A\left(\lambda_{j}, 0\right)\right\|+\left\|A\left(\lambda_{j}^{+}, 0\right)\right\|\right)+\int_{0}^{T} F_{\beta}(s) d s \leq \gamma \beta+\int_{0}^{T} P(t) d t \\
\text { and } \int_{0}^{T} L_{\beta}(t) d t<\frac{1}{2}, \quad \text { for all } \beta>0 .
\end{gathered}
$$

Hence, it follows by Theorem 5.1.10 that the equation (5.15) admits a unique $T$-periodic solution in $X_{\beta}=\left\{x \in C_{T}\left(\mathbb{R}, \mathbb{R}^{n}\right):|x|_{T} \leq \beta\right\}$ for every $\beta \geq \frac{\int_{0}^{T} P(t) d t}{\frac{1}{2}-\gamma}$.

The next example concerns a perturbed Van Der Pol equation.

Example 5.2.2. Consider the following perturbed Van Der Pol equation given by

$$
z^{\prime \prime}-\varepsilon_{2}\left(1-z^{2}\right) z^{\prime}+z-\varepsilon_{1} \frac{d}{d t} g(t, z(t-r))=0,
$$

almost everywhere in $\mathbb{R}$, where $r \in \mathbb{R}$ and $\varepsilon_{1}, \varepsilon_{2}$ are small positive parameters.

Assume that $g: \mathbb{R} \times C(\mathbb{R}, \mathbb{R}) \rightarrow \mathbb{R}$ is $T$-periodic in the first variable and for every $y \in$ $C_{T}(\mathbb{R}, \mathbb{R})$, the mapping $t \mapsto g\left(t, y_{t}\right)$ is left-continuous with $\left|\Lambda_{g}^{[0, T)}\right|=m$ and almost differentiable in $\mathbb{R}$.

Moreover, assume that there is a constant $\xi>0$ such that

$$
0<\varepsilon_{1}<\frac{1}{2(2 m+1) \xi} \quad \text { and } \quad|g(t, z)-g(t, w)| \leq \xi|z-w|_{T}
$$

for all $(t, z),(t, w) \in \mathbb{R} \times C_{T}(\mathbb{R}, \mathbb{R})$.

Let us consider the norm $\|D\|=\max _{1 \leq i \leq n} \sum_{j=1}^{n}\left|d_{i j}\right|$ for an $n \times n$-matrix $D=\left(d_{i j}\right)$. By choosing $x_{1}=z$ and $x_{2}=x_{1}^{\prime}$, we can transform equation (5.16) into

$$
\left(\begin{array}{c}
x_{1}^{\prime} \\
x_{2}^{\prime}
\end{array}\right)=\left(\begin{array}{cc}
0 & 1 \\
-1 & \varepsilon_{2}
\end{array}\right)\left(\begin{array}{c}
x_{1} \\
x_{2}
\end{array}\right)-\varepsilon_{2}\left(\begin{array}{c}
0 \\
x_{2}(t) x_{1}^{2}(t)
\end{array}\right)+\varepsilon_{1}\left(\begin{array}{c}
0 \\
\frac{d}{d t} g\left(t, x_{1}(t-r)\right)
\end{array}\right) \text {. }
$$


Let $f, A: \mathbb{R} \times C\left(\mathbb{R}, \mathbb{R}^{2}\right) \rightarrow \mathbb{R}^{2}$ be defined by

$$
\begin{gathered}
A(t, \phi)=\left(\begin{array}{c}
0 \\
\varepsilon_{1} g\left(t, \phi_{1}(-r)\right)
\end{array}\right) \text { and } \\
f(t, \phi)=\left(\begin{array}{cc}
0 & 1 \\
-1 & \varepsilon_{2}
\end{array}\right)\left(\begin{array}{c}
\phi_{1}(0) \\
\phi_{2}(0)
\end{array}\right)+\left(\begin{array}{c}
0 \\
-\varepsilon_{2} \phi_{2}(0) \phi_{1}^{2}(0)
\end{array}\right),
\end{gathered}
$$

for all $\phi=\left[\phi_{1}, \phi_{2}\right]^{t} \in C\left(\mathbb{R}, \mathbb{R}^{2}\right)$. Let $C=\left(\begin{array}{cc}0 & 1 \\ -1 & \varepsilon_{2}\end{array}\right)$.

Observe that $\alpha=\sup _{t \in[0, T]}|g(t, 0)|<\infty$, since the mapping $\mathbb{R} \ni t \mapsto g(t, 0)$ is a regulated function.

Let $\gamma_{1}, \gamma_{2} \in \mathbb{R}$ be such that $0<\gamma_{1}+\gamma_{2} \leq 1$ and $\left(2+\varepsilon_{2}\right) T<\frac{\gamma_{2}}{2}$. In addition, consider a constant $\beta>0$ satisfying

$$
\frac{2(2 m+1) \varepsilon_{1} \alpha}{\gamma_{1}} \leq \beta \leq \sqrt{\frac{\gamma_{2}-\left(4+2 \varepsilon_{2}\right) T}{2 \varepsilon_{2} T}} .
$$

Then, we claim that the perturbed Van Der Pol equation (5.16) admits at least one periodic solution in $X_{\beta}=\left\{x \in C_{T}\left(\mathbb{R}, \mathbb{R}^{2}\right):|x|_{T} \leq \beta\right\}$ provided that the above conditions hold.

In fact, at first let us verify that conditions (J1)-(J5) hold.

Conditions (J1)-(J2) follow by our assumptions and from the fact that if $x=\left[x_{1}, x_{2}\right]^{t} \in$ $C_{T}\left(\mathbb{R}, \mathbb{R}^{2}\right)$ then the mapping $\mathbb{R} \ni t \mapsto f\left(t, x_{t}\right)$ is locally Lebesgue integrable over $\mathbb{R}$, since $x$ is bounded.

Note that (J3) holds, because given $\mu>0, x=\left[x_{1}, x_{2}\right]^{t}, y=\left[y_{1}, y_{2}\right]^{t} \in C_{T}\left(\mathbb{R}, \mathbb{R}^{2}\right)$ such that $|x|_{T} \leq \mu$ and $|y|_{T} \leq \mu$, and $t \in[0, T]$, we have

$$
\begin{aligned}
\left\|A\left(t, x_{t}\right)-A\left(t, y_{t}\right)\right\| & \leq \varepsilon_{1}\left|g\left(t, x_{1}(t-r)\right)-g\left(t, y_{1}(t-r)\right)\right| \\
& \leq \varepsilon_{1} \xi|x-y|_{T} \\
& =\gamma_{\mu}\left(|x-y|_{T}\right),
\end{aligned}
$$

where $\gamma_{\mu}(t)=\varepsilon_{1} \xi$, for all $t \geq 0$. Thus, by the choice of $\varepsilon_{1}$ we get $\varepsilon_{1} \xi<\frac{1}{4 m+2}$.

Now, to check the validity of condition (J4), let $\mu>0$ be given. Now, let $x=\left[x_{1}, x_{2}\right]^{t} \in$ $C_{T}\left(\mathbb{R}, \mathbb{R}^{2}\right)$ be such that $|x|_{T} \leq \mu$ and $t_{1}, t_{2} \in[0, T], t_{1} \leq t_{2}$. Then

$$
\begin{aligned}
\left\|\int_{t_{1}}^{t_{2}} f\left(t, x_{t}\right) d t\right\| & \leq \int_{t_{1}}^{t_{2}}\|C\||x|_{T} d t+\int_{t_{1}}^{t_{2}}\left\|\left(\begin{array}{c}
0 \\
-\varepsilon_{2} x_{2}(t) x_{1}^{2}(t)
\end{array}\right)\right\| d t \\
& \leq \int_{t_{1}}^{t_{2}}\left[\left(2+\varepsilon_{2}\right) \mu+\varepsilon_{2}|x|_{T}|x|_{T}^{2}\right] d t \\
& \leq \int_{t_{1}}^{t_{2}}\left[\left(2+\varepsilon_{2}\right) \mu+\varepsilon_{2} \mu^{3}\right] d t \\
& =\int_{t_{1}}^{t_{2}} F_{\mu}(t) d t
\end{aligned}
$$


where $F_{\mu}(t)=\left(2+\varepsilon_{2}\right) \mu+\varepsilon_{2} \mu^{3}$ for all $t \in \mathbb{R}$.

Finally, let us check that condition (J5) is satisfied. Let $\mu>0$ and $x=\left[x_{1}, x_{2}\right]^{t}, y=$ $\left[y_{1}, y_{2}\right]^{t} \in C_{T}\left(\mathbb{R}, \mathbb{R}^{2}\right)$ be such that $|x|_{T} \leq \mu$ and $|y|_{T} \leq \mu$. Thus, for all $t_{1}, t_{2} \in[0, T], t_{1} \leq t_{2}$, we get

$$
\begin{aligned}
& \left\|\int_{t_{1}}^{t_{2}}\left[f\left(t, x_{t}\right)-f\left(t, y_{t}\right)\right] d t\right\| \leq \\
& \leq \int_{t_{1}}^{t_{2}}\|C\||x-y|_{T} d t+\int_{t_{1}}^{t_{2}}\left\|\left(\begin{array}{c}
0 \\
-\varepsilon_{2} x_{2}(t) x_{1}^{2}(t)+\varepsilon_{2} y_{2}(t) y_{1}^{2}(t)
\end{array}\right)\right\| d t \\
& \leq \int_{t_{1}}^{t_{2}}\|C\||x-y|_{T} d t+\int_{t_{1}}^{t_{2}}\left|\varepsilon_{2} x_{2}(t) x_{1}^{2}(t)-\varepsilon_{2} y_{2}(t) y_{1}^{2}(t)\right| d t \\
& \leq \int_{t_{1}}^{t_{2}}\|C\||x-y|_{T} d t+\int_{t_{1}}^{t_{2}} \varepsilon_{2}\left\|\left(\begin{array}{ll}
x_{2}(t)\left(x_{1}(t)+y_{1}(t)\right) & \left.y_{1}^{2}(t)\right)
\end{array}\right)\left(\begin{array}{l}
x_{1}(t)-y_{1}(t) \\
x_{2}(t)-y_{2}(t)
\end{array}\right)\right\| d t \\
& \leq \int_{t_{1}}^{t_{2}}\|C\|\|x-y\|_{T} d t+\varepsilon_{2} \int_{t_{1}}^{t_{2}} 2 \mu^{2}\|x(t)-y(t)\| d t \\
& \leq \int_{t_{1}}^{t_{2}}\left(2+\varepsilon_{2}\right)|x-y|_{T} d t+\int_{t_{1}}^{t_{2}} 2 \mu^{2} \varepsilon_{2}|x-y|_{T} d t \\
& =\int_{t_{1}}^{t_{2}} L_{\mu}(t)|x-y|_{T} d t,
\end{aligned}
$$

where $L_{\mu}(t)=2+\varepsilon_{2}+2 \varepsilon_{2} \mu^{2}$ for all $t \in \mathbb{R}$.

By the choices of $\varepsilon_{1}, \varepsilon_{2}, T$ and $\beta$, we have

$$
\begin{gathered}
\sup _{t \in[0, T]}\|A(t, 0)\|+\sum_{0<\lambda_{j}<T}\left(\left\|A\left(\lambda_{j}, 0\right)\right\|+\left\|A\left(\lambda_{j}^{+}, 0\right)\right\|\right)+\int_{0}^{T} F_{\beta}(s) d s \\
\leq(2 m+1) \varepsilon_{1} \alpha+\int_{0}^{T} F_{\beta}(s) d s \leq\left(\gamma_{1}+\gamma_{2}\right) \frac{\beta}{2} \leq \frac{\beta}{2} .
\end{gathered}
$$

Therefore, by Theorem 5.1.9, the pertubed Van Der Pol equation (5.16) has at least one $T$-periodic solution in $X_{\beta}$.

If, in addition, we also add the extra condition

$$
\beta<\sqrt{\frac{1-2 T\left(2+\varepsilon_{2}\right)}{4 \varepsilon_{2} T}}
$$

then, we conclude that

$$
\int_{0}^{T} L_{\beta}(t) d t=\int_{0}^{T}\left[2+\varepsilon_{2}+2 \varepsilon_{2} \beta^{2}\right] d t \leq T\left(2+\varepsilon_{2}\right)+2 \varepsilon_{2} \beta^{2} T<\frac{1}{2} .
$$

Therefore, by Theorem 5.1.10, the pertubed Van Der Pol equation (5.16) has a unique $T$-periodic solution in $X_{\beta}$.

In the next example, we present sufficient conditions to obtain periodic solutions for a neutral Volterra integro-differential equation type. 
Example 5.2.3. Consider the neutral Volterra integro-differential equation with infinite delay

$$
\frac{d}{d t}\left[x(t)-\int_{-\infty}^{t} D(t, s) x(s) d s\right]=a(t) x^{k}(t)+\int_{-\infty}^{t} C(t, s) x(s) d s+p(t),
$$

almost everywhere in $\mathbb{R}$, under the following conditions:

(a) $k \in \mathbb{N}$ and there exists $T>0$ such that $a(t+T)=a(t), C(t+T, s+T)=C(t, s)$ and $D(t+T, s+T)=D(t, s)$, for all $t, s \in \mathbb{R}$;

(b) for each $x \in C_{T}\left(\mathbb{R}, \mathbb{R}^{n}\right)$, the mappings $s \mapsto D(t, s) x(s)$ and $s \mapsto C(t, s) x(s)$ are Perron integrable over $(-\infty, t]$ with $t \in \mathbb{R}$, the functions $t \mapsto a(t) x^{k}(t), t \mapsto p(t)$ and $t \mapsto \int_{-\infty}^{t} C(t, s) x(s) d s$ are locally Perron integrable over $\mathbb{R}$, and for each $s \in \mathbb{R}$, the function $t \mapsto D(t, s)$ is continuous on $\mathbb{R}$;

(c) there exist a constant $0<d^{*}<\frac{1}{2}$ and a non-negative locally Perron integrable function $D^{*}: \mathbb{R} \rightarrow \mathbb{R}_{+}$such that $\int_{-\infty}^{T} D^{*}(s) d s \leq d^{*}$ and, for each $t \in[0, T]$, the inequality

$$
\left\|\int_{-\infty}^{t} D(t, s)\left[z_{2}(s)-z_{1}(s)\right] d s\right\| \leq \int_{-\infty}^{t} D^{*}(s)\left|z_{2}-z_{1}\right|_{T} d s,
$$

holds whenever $z_{1}, z_{2} \in C_{T}\left(\mathbb{R}, \mathbb{R}^{n}\right)$;

(d) there are non-negative locally Perron integrable functions $M, N: \mathbb{R} \rightarrow \mathbb{R}_{+}$such that, for all $t_{1}, t_{2} \in[0, T], t_{1} \leq t_{2}$, we have

$$
\begin{gathered}
\left\|\int_{t_{1}}^{t_{2}} a(t)\left[\phi^{k}(t)-\psi^{k}(t)\right] d t\right\| \leq \int_{t_{1}}^{t_{2}} M(t)\left|\phi^{k}-\psi^{k}\right|_{T} d t \quad \text { and } \\
\left\|\int_{t_{1}}^{t_{2}}\left(\int_{-\infty}^{t} C(t, s)[\phi(s)-\psi(s)] d s\right) d t\right\| \leq \int_{t_{1}}^{t_{2}} N(t)|\phi-\psi|_{T} d t,
\end{gathered}
$$

whenever $\phi, \psi \in C_{T}\left(\mathbb{R}, \mathbb{R}^{n}\right)$;

(e) $p: \mathbb{R} \rightarrow \mathbb{R}^{n}$ is $T$-periodic and there exists a non-negative locally Perron integrable function $Q: \mathbb{R} \rightarrow \mathbb{R}_{+}$such that

$$
\left\|\int_{t_{1}}^{t_{2}} p(t) d t\right\| \leq \int_{t_{1}}^{t_{2}} Q(t) d t
$$

for all $t_{1}, t_{2} \in[0, T], t_{1} \leq t_{2}$.

Let $f, A: \mathbb{R} \times C\left(\mathbb{R}, \mathbb{R}^{n}\right) \rightarrow \mathbb{R}^{n}$ be defined by

$$
f(t, \phi)=a(t) \phi^{k}(0)+\int_{-\infty}^{t} C(t, s) \phi(s-t) d s+p(t)
$$

and

$$
A(t, \phi)=\int_{-\infty}^{t} D(t, s) \phi(s-t) d s,
$$

for all $t \in \mathbb{R}$ and all $\phi \in C\left(\mathbb{R}, \mathbb{R}^{n}\right)$. 
Let us show that conditions (J1)-(J5) hold. Conditions (J1)-(J2) follow immediately from the assumptions (a), (b) and (e).

To show that condition (J3) is satisfied, let $t \in[0, T]$ and $x, y \in C_{T}\left(\mathbb{R}, \mathbb{R}^{n}\right)$. Condition (c) implies that

$$
\begin{aligned}
\left\|A\left(t, x_{t}\right)-A\left(t, y_{t}\right)\right\| & =\left\|\int_{-\infty}^{t} D(t, s) x(s) d s-\int_{-\infty}^{t} D(t, s) y(s) d s\right\| \\
& \leq \int_{-\infty}^{t} D^{*}(s)|x-y|_{T} d s \leq \int_{-\infty}^{T} D^{*}(s)|x-y|_{T} d s \\
& \leq d^{*}|x-y|_{T} .
\end{aligned}
$$

Thus, condition (J3) holds, with $m=0$, by taking $\gamma(s)=d^{*} s$ for all $s \geq 0$, which does not depend on the choice of $\mu>0$.

To check condition (J4), take $\mu>0$ arbitrarily. Let $x \in C_{T}\left(\mathbb{R}, \mathbb{R}^{n}\right)$ be such that $|x|_{T} \leq \mu$. Set $F_{\mu}(t)=M(t) \mu^{k}+N(t) \mu+Q(t)$, for $t \in \mathbb{R}$. By (d) and (e), we get

$$
\begin{aligned}
\left\|\int_{t_{1}}^{t_{2}} f\left(t, x_{t}\right) d t\right\| & \leq\left\|\int_{t_{1}}^{t_{2}} a(t) x^{k}(t) d t\right\|+\left\|\int_{t_{1}}^{t_{2}}\left(\int_{-\infty}^{t} C(t, s) x(s) d s\right) d t\right\| \\
& +\left\|\int_{t_{1}}^{t_{2}} p(t) d t\right\| \\
& \leq \int_{t_{1}}^{t_{2}} M(t)|x|_{T}^{k} d t+\int_{t_{1}}^{t_{2}} N(t)|x|_{T} d t+\int_{t_{1}}^{t_{2}} Q(t) d t \\
& \leq \int_{t_{1}}^{t_{2}}\left[M(t) \mu^{k}+N(t) \mu+Q(t)\right] d t=\int_{t_{1}}^{t_{2}} F_{\mu}(t) d t,
\end{aligned}
$$

for all $t_{1}, t_{2} \in[0, T], t_{1} \leq t_{2}$.

Finally, we assert that condition (J5) holds. Indeed, let $x, y \in C_{T}\left(\mathbb{R}, \mathbb{R}^{n}\right)$ be such that $|x|_{T} \leq \mu$ and $|y|_{T} \leq \mu$, and $t_{1}, t_{2} \in[0, T]$ be such that $t_{1} \leq t_{2}$. Using condition (d), we obtain

$$
\begin{aligned}
& \left\|\int_{t_{1}}^{t_{2}}\left[f\left(t, x_{t}\right)-f\left(t, y_{t}\right)\right] d t\right\| \leq\left\|\int_{t_{1}}^{t_{2}} a(t)\left[x^{k}(t)-y^{k}(t)\right] d t\right\| \\
& +\left\|\int_{t_{1}}^{t_{2}}\left(\int_{-\infty}^{t} C(t, s)[x(s)-y(s)] d s\right) d t\right\| \\
& \leq \int_{t_{1}}^{t_{2}} M(t)\left|x^{k}-y^{k}\right|_{T} d t+\int_{t_{1}}^{t_{2}} N(t)|x-y|_{T} d t \\
& \leq \int_{t_{1}}^{t_{2}} M(t)|x-y|_{T} \sum_{j=0}^{k-1}|x|_{T}^{j}|y|_{T}^{k-1-j} d t+\int_{t_{1}}^{t_{2}} N(t)|x-y|_{T} d t \\
& \leq \int_{t_{1}}^{t_{2}}\left[M(t) k \mu^{k-1}+N(t)\right]|x-y|_{T} d t .
\end{aligned}
$$

Consequently, it is enough to take $L_{\mu}(t)=M(t) k \mu^{k-1}+N(t), t \in \mathbb{R}$.

Now, let $\gamma_{1}, \gamma_{2} \in \mathbb{R}$ be such that $0<\gamma_{1}+\gamma_{2} \leq 1$. Assume that $k>1$. If

$$
\int_{0}^{T} N(t) d t<\frac{\gamma_{1}}{2} \text { and } \frac{2}{\gamma_{2}} \int_{0}^{T} Q(t) d t \leq \beta \leq\left(\frac{\gamma_{1}-2 \int_{0}^{T} N(t) d t}{2 \int_{0}^{T} M(t) d t}\right)^{\frac{1}{k-1}}
$$


then, using the fact that $A(t, 0)=0$, we get

$$
\begin{aligned}
& \sup _{t \in[0, T]}\|A(t, 0)\|+\sum_{0<\lambda_{j}<T}\left(\left\|A\left(\lambda_{j}, 0\right)\right\|+\left\|A\left(\lambda_{j}^{+}, 0\right)\right\|\right)+\int_{0}^{T} F_{\beta}(s) d s \\
& =\int_{0}^{T}\left[M(s) \beta^{k-1}+N(s)\right] \beta d s+\int_{0}^{T} Q(s) d s \\
& \leq\left(\gamma_{1}+\gamma_{2}\right) \frac{\beta}{2} \leq \frac{\beta}{2} .
\end{aligned}
$$

Therefore, by Theorem 5.1.9, the neutral Volterra integro-differential equation with infinite delay (5.17) has at least one $T$-periodic solution in $X_{\beta}=\left\{x \in C_{T}\left(\mathbb{R}, \mathbb{R}^{n}\right):|x|_{T} \leq \beta\right\}$. Also, if we add the extra condition

$$
\beta<\left(\frac{\frac{1}{2}-\int_{0}^{T} N(t) d t}{k \int_{0}^{T} M(t) d t}\right)^{\frac{1}{k-1}}
$$

then

$$
\int_{0}^{T} L_{\beta}(t) d t=\int_{0}^{T}\left[M(t) k \beta^{k-1}+N(t)\right] d t<\frac{1}{2}
$$

and, by Theorem 5.1.10, the neutral Volterra integro-differential equation with infinite delay (5.17) has a unique $T$-periodic solution in $X_{\beta}$.

In the case that $k=1$, if

$$
\int_{0}^{T} M(t) d t+\int_{0}^{T} N(t) d t \leq \frac{\gamma_{1}}{2} \text { and } \beta \geq \frac{2}{\gamma_{2}} \int_{0}^{T} Q(t) d t
$$

then the neutral Volterra integro-differential equation with infinite delay (5.17) has at least one $T$-periodic solution in $X_{\beta}$. In addition, if

$$
\int_{0}^{T}[M(t)+N(t)] d t<\frac{1}{2},
$$

then the neutral Volterra integro-differential equation with infinite delay (5.17) has a unique $T$-periodic solution in $X_{\beta}$.

Next, we consider a particular case of Example 5.2.3.

Example 5.2.4. Consider the real function

$$
g(t)= \begin{cases}2 t \sin \left(\frac{1}{t}\right)-\cos \left(\frac{1}{t}\right), & \text { if } 0<t \leq 2 \pi \\ 0, & \text { if } t=0\end{cases}
$$

By Example 2.4.24, $g$ is a Perron integrable function on $[0,2 \pi]$, but it is neither Riemann nor Lebesgue integrable. Define $p: \mathbb{R} \rightarrow \mathbb{R}$ by

$$
p(t)=g(t-2 \pi q), \quad \text { for } \quad 2 \pi q<t \leq 2 \pi(q+1), q \in \mathbb{Z} .
$$

Now, consider the following NFDE

$$
\frac{d}{d t}\left[x(t)-\int_{-\infty}^{t} \alpha e^{-t+s} x(s) d s\right]=\frac{\sin (t)}{4(8 \pi)^{3}} x^{k}(t)+\int_{-\infty}^{t} \frac{1}{16 \pi} e^{-t+s} x(s) d s+\eta p(t),
$$


almost everywhere in $\mathbb{R}$, which is a particular case of Example 5.2.3, where the functions $D(t, s)=\alpha e^{-t+s}, a(t)=\frac{\sin (t)}{4(8 \pi)^{3}}$ and $C(t, s)=\frac{1}{16 \pi} e^{-t+s}$, for all $t, s \in \mathbb{R}$. Suppose that $0<\alpha<\frac{e^{-2 \pi}}{2}$ and $\eta>0$.

Let us show that conditions (a)-(e) of Example 5.2.3 hold.

Note that $C(t+2 \pi, s+2 \pi)=C(t, s), D(t+2 \pi, s+2 \pi)=D(t, s)$, for all $t, s \in \mathbb{R}$, and $a(t+2 \pi)=a(t)$, for all $t \in \mathbb{R}$. Thus, condition (a) holds.

Let $x \in C_{2 \pi}(\mathbb{R}, \mathbb{R})$. For each $s \in \mathbb{R}$, the maps $s \mapsto \alpha e^{-t+s}$ and $s \mapsto \frac{1}{16 \pi} e^{-t+s}$ are locally Perron integrable functions over $\mathbb{R}$. Also, the maps $t \mapsto \frac{\sin (t)}{4(8 \pi)^{3}}$ and $t \mapsto p(t)$ are locally Perron integrable over $\mathbb{R}$, because these maps are locally Lebesgue integrable over $\mathbb{R}$. Hence, condition (b) holds.

To verify that condition (c) holds, we take $d^{*}=\alpha e^{2 \pi}$ and a non-negative locally Lesbegue integrable function $D^{*}: \mathbb{R} \rightarrow \mathbb{R}_{+}$given by $D^{*}(t)=\alpha e^{t}$. Let $t \in[0,2 \pi]$ and $x, y \in C_{2 \pi}(\mathbb{R}, \mathbb{R})$. Then

$$
\begin{aligned}
\left|\int_{-\infty}^{t} \alpha e^{-t+s}[x(s)-y(s)] d s\right| & \leq \int_{-\infty}^{t} \alpha e^{-t+s}|x(s)-y(s)| d s \\
& \leq \int_{-\infty}^{t} \alpha e^{s}|x-y|_{2 \pi} d s .
\end{aligned}
$$

We claim that condition (d) is satisfied with $M(t)=\frac{1}{4(8 \pi)^{3}}$ and $N(t)=\frac{1}{16 \pi}, t \in \mathbb{R}$. Indeed, let $t_{1}, t_{2} \in[0,2 \pi], t_{1} \leq t_{2}$, and $x, y \in C_{2 \pi}(\mathbb{R}, \mathbb{R})$. Then

$$
\begin{aligned}
\left|\int_{t_{1}}^{t_{2}} a(t)\left[x^{k}(t)-y^{k}(t)\right] d t\right| & =\left|\int_{t_{1}}^{t_{2}} \frac{\sin (t)}{4(8 \pi)^{3}}\left[x^{k}(t)-y^{k}(t)\right] d t\right| \\
& \leq \int_{t_{1}}^{t_{2}} \frac{|\sin (t)|}{4(8 \pi)^{3}}\left|x^{k}(t)-y^{k}(t)\right| d t \\
& \leq \int_{t_{1}}^{t_{2}} \frac{1}{4(8 \pi)^{3}}\left|x^{k}-y^{k}\right|_{2 \pi} d t \\
& \leq \int_{t_{1}}^{t_{2}} M(t)\left|x^{k}-y^{k}\right|_{2 \pi} d t
\end{aligned}
$$

and

$$
\begin{aligned}
& \left|\int_{t_{1}}^{t_{2}}\left(\int_{-\infty}^{t} C(t, s)[x(s)-y(s)] d s\right) d t\right|=\left|\int_{t_{1}}^{t_{2}}\left(\int_{-\infty}^{t} \frac{1}{16 \pi} e^{-t+s}[x(s)-y(s)] d s\right) d t\right| \\
& \leq \int_{t_{1}}^{t_{2}}\left|\int_{-\infty}^{t} \frac{1}{16 \pi} e^{-t+s}[x(s)-y(s)] d s\right| d t \leq \int_{t_{1}}^{t_{2}}\left(\int_{-\infty}^{t} \frac{1}{16 \pi} e^{-t+s}|x(s)-y(s)| d s\right) d t \\
& \leq \int_{t_{1}}^{t_{2}} \frac{1}{16 \pi}\left(\int_{-\infty}^{t} e^{-t+s} d s\right)|x-y|_{2 \pi} d t=\int_{t_{1}}^{t_{2}} N(t)|x-y|_{2 \pi} d t .
\end{aligned}
$$

Finally, condition (e) holds since the function $p(t)=g(t-2 \pi q), t \in \mathbb{R}$, is $2 \pi$-periodic and the map $t \mapsto p(t)$ is locally Perron integrable over $\mathbb{R}$. Further, if we take $Q(t)=\eta(1+4 \pi)$, for all $t \in \mathbb{R}$, then

$$
|p(\tau)|=|g(\tau)| \leq 2 \tau+1 \leq 4 \pi+1, \text { for all } \tau \in[0,2 \pi] .
$$


Then, for all $t_{1}, t_{2} \in[0,2 \pi], t_{1} \leq t_{2}$, and $x \in C_{2 \pi}(\mathbb{R}, \mathbb{R})$, we have

$$
-\int_{t_{1}}^{t_{2}} \eta(4 \pi+1) d t \leq \int_{t_{1}}^{t_{2}} \eta p(t) d t \leq \int_{t_{1}}^{t_{2}} \eta(4 \pi+1) d t
$$

that is,

$$
\left|\int_{t_{1}}^{t_{2}} \eta p(t) d t\right| \leq \int_{t_{1}}^{t_{2}} \eta(4 \pi+1) d t
$$

Now, let $\gamma_{1}, \gamma_{2} \in \mathbb{R}$ be such that $0<\gamma_{1}+\gamma_{2} \leq 1$. According to Example 5.2.3, if $k>1$,

$$
\gamma_{1}>\frac{1}{4} \text { and } \quad \frac{4 \pi \eta(1+4 \pi)}{\gamma_{2}}<\left(2(8 \pi)^{2}\left(4 \gamma_{1}-1\right)\right)^{\frac{1}{k-1}},
$$

then the NFDE (5.20) admits a $2 \pi$-periodic solution in $X_{\beta}=\left\{x \in C_{T}(\mathbb{R}, \mathbb{R}):|x|_{T} \leq \beta\right\}$ for

$$
\frac{4 \pi \eta(1+4 \pi)}{\gamma_{2}} \leq \beta \leq\left(2(8 \pi)^{2}\left(4 \gamma_{1}-1\right)\right)^{\frac{1}{k-1}}
$$

In addition, if $\beta<\left(\frac{6(8 \pi)^{2}}{k}\right)^{\frac{1}{k-1}}$, then the NFDE (5.20) admits a unique $2 \pi$-periodic solution in $X_{\beta}$.

Now, if $k=1$ and the following inequalities hold

$$
\gamma_{1}>\frac{1}{8^{3} \pi^{2}}+\frac{1}{4} \quad \text { and } \quad \beta \geq \frac{4}{\gamma_{2}} \pi \eta(1+4 \pi),
$$

then the NFDE (5.20) admits a unique $2 \pi$-periodic solution in $X_{\beta}$.

The last example of this section involves an equation where the neutral term $A(t, \phi)$ is nonlinear.

Example 5.2.5. Consider the NFDE

$$
\frac{d}{d t}(x(t)+a g(x(t-\tau)))=b x(t-r)+p(t),
$$

almost everywhere in $\mathbb{R}$, under the following conditions:

(a) $\tau, r \in \mathbb{R}, p \in G^{-}\left(\mathbb{R}, \mathbb{R}^{n}\right), g: \mathbb{R}^{n} \rightarrow \mathbb{R}^{n}$ is $K$-Lipschitz and differentiable almost everywhere on $\mathbb{R}$;

(b) $a, b \in \mathbb{R}$ and $|a|<\frac{1}{2 K}$;

(c) the mapping $\mathbb{R} \ni t \mapsto p(t)$ is locally Perron integrable over $\mathbb{R}, p(t+T)=p(t)$ for every $t \in \mathbb{R}$ and $T>0$, and there is a non-negative locally Perron integrable function $P: \mathbb{R} \rightarrow \mathbb{R}_{+}$ such that, for all $t_{1}, t_{2} \in[0, T], t_{1} \leq t_{2}$,

$$
\left\|\int_{t_{1}}^{t_{2}} p(t) d t\right\| \leq \int_{t_{1}}^{t_{2}} P(t) d t
$$


Let $A, f: \mathbb{R} \times C\left(\mathbb{R}, \mathbb{R}^{n}\right) \rightarrow \mathbb{R}^{n}$ be given by

$$
A(t, \phi)=-a g(\phi(-\tau)) \quad \text { and } \quad f(t, \phi)=b \phi(-r)+p(t)
$$

for all $t \in \mathbb{R}$ and $\phi \in C\left(\mathbb{R}, \mathbb{R}^{n}\right)$.

In what follows, we verify that conditions (J1)-(J5) hold.

Conditions (J1)-(J2) follow immediately from the hypotheses (a) and (c).

To verify that (J3) is satisfied, take $\mu>0$. Notice that, given $x, y \in C_{T}\left(\mathbb{R}, \mathbb{R}^{n}\right)$ such that $|x|_{T} \leq \mu,|y|_{T} \leq \mu$, it follows from condition (a) that

$$
\begin{aligned}
\left\|A\left(t, x_{t}\right)-A\left(t, y_{t}\right)\right\| & =\|a g(y(t-\tau))-a g(x(t-\tau))\| \\
& \leq|a| K|x-y|_{T} \\
& =\gamma_{\mu}\left(|x-y|_{T}\right)
\end{aligned}
$$

for all $t \in[0, T]$, where $\gamma_{\mu}(s)=|a| K s$ for all $s \geq 0$. By item (b), we have $|a| K<\frac{1}{2}$ (in this case $m=0)$.

Now, let us check that condition (J4) holds. Let $\mu>0$ be given. If $x \in C_{T}\left(\mathbb{R}, \mathbb{R}^{n}\right)$ is such that $|x|_{T} \leq \mu$, then conditions (a)-(c) imply

$$
\begin{aligned}
\left\|\int_{t_{1}}^{t_{2}} f\left(t, x_{t}\right) d t\right\| & \leq \int_{t_{1}}^{t_{2}}|b||x|_{T} d t+\left\|\int_{t_{1}}^{t_{2}} p(t) d t\right\| \\
& \leq \int_{t_{1}}^{t_{2}}|b| \mu d t+\int_{t_{1}}^{t_{2}} P(t) d t \\
& =\int_{t_{1}}^{t_{2}} F_{\mu}(t) d t,
\end{aligned}
$$

for all $t_{1}, t_{2} \in[0, T], t_{1} \leq t_{2}$, with $F_{\mu}(t)=|b| \mu+P(t), t \in \mathbb{R}$.

Finally, we claim that (J5) holds. Indeed, taking $t_{1}, t_{2} \in[0, T]$ with $t_{1} \leq t_{2}$, and using condition (a), we have

$$
\begin{aligned}
\left\|\int_{t_{1}}^{t_{2}}\left[f\left(t, x_{t}\right)-f\left(t, y_{t}\right)\right] d t\right\| & =\left\|\int_{t_{1}}^{t_{2}} b[x(t-r)-y(t-r)] d t\right\| \\
& \leq \int_{t_{1}}^{t_{2}}|b||x-y|_{T} d t
\end{aligned}
$$

whenever $x, y \in C_{T}\left(\mathbb{R}, \mathbb{R}^{n}\right)$ are such that $|x|_{T} \leq \mu$ and $|y|_{T} \leq \mu$. Hence, considering $L_{\mu}(t)=|b|$, $t \in \mathbb{R}$, we conclude that (J5) holds.

Therefore, taking $b$ and $T$ such that $|b| T<\frac{1}{2}$ and $\beta>0$ satisfying

$$
\beta \geq \frac{|a|\|g(0)\|+\int_{0}^{T} P(t) d t}{\frac{1}{2}-|b| T},
$$

we conclude that

$$
\int_{0}^{T} L_{\beta}(t) d t=|b| T<\frac{1}{2}
$$


and

$$
\begin{aligned}
& \sup _{t \in[0, T]}\|A(t, 0)\|+\sum_{0<\lambda_{j}<T}\left(\left\|A\left(\lambda_{j}, 0\right)\right\|+\left\|A\left(\lambda_{j}^{+}, 0\right)\right\|\right)+\int_{0}^{T} F_{\beta}(s) d s \\
& =|a|\|g(0)\|+\int_{0}^{T}[|b| \beta+P(t)] d t \\
& \leq|a|\|g(0)\|+|b| T \beta+\int_{0}^{T} P(t) d t \leq \frac{\beta}{2} .
\end{aligned}
$$

Therefore, by Theorem 5.1.10, the equation (5.21) has a unique $T$-periodic solution in $X_{\beta}=$ $\left\{x \in C_{T}\left(\mathbb{R}, \mathbb{R}^{n}\right):|x|_{T} \leq \beta\right\}$. 
CHAPTER

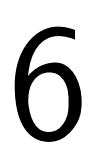

\section{NEUTRAL FUNCTIONAL DIFFERENTIAL EQUATIONS WITH IMPULSES}

In this chapter, we establish sufficient conditions for the existence and uniqueness of periodic solutions for the following class of neutral functional differential equations with impulses (impulsive NFDEs for short)

$$
\begin{cases}\frac{d}{d t}\left(x(t)-B\left(t, x_{t}\right)\right)=g\left(t, x_{t}\right), & t \neq t_{k}, k \in \mathbb{Z}, \\ x\left(t_{k}^{+}\right)-x\left(t_{k}\right)=b_{k} x\left(t_{k}\right), & k \in \mathbb{Z},\end{cases}
$$

where the moments of impulse effects $\left\{\ldots, t_{-k}, \ldots, t_{-1}, t_{0}, t_{1}, \ldots, t_{k}, \ldots\right\} \subset \mathbb{R}$ are such that

$$
\ldots<t_{-k}<\ldots<t_{-2}<t_{-1}<t_{0}<t_{1}<t_{2}<\ldots<t_{k}<\ldots
$$

and $\lim _{k \rightarrow \pm \infty} t_{k}= \pm \infty$

\subsection{Existence of periodic solutions}

A solution of the impulsive NFDE (6.1) is defined as follows.

Definition 6.1.1. A function $x: \mathbb{R} \rightarrow \mathbb{R}^{n}$ is said to be a solution of the impulsive NFDE (6.1), if the following conditions hold:

(i) $x \in C\left(\left(t_{j}, t_{j+1}\right], \mathbb{R}^{n}\right)$, that is, $x$ is continuous on each interval $\left(t_{j}, t_{j+1}\right], j \in \mathbb{Z}$;

(ii) $x$ satisfies the NFDE $\frac{d}{d t}\left(x(t)-B\left(t, x_{t}\right)\right)=g\left(t, x_{t}\right)$ almost everywhere in $\mathbb{R} \backslash\left\{t_{k}: k \in \mathbb{Z}\right\}$;

(iii) $x\left(t_{k}^{+}\right)-x\left(t_{k}\right)=b_{k} x\left(t_{k}\right), k \in \mathbb{Z}$.

In order to find periodic solutions of the impulsive NFDE (6.1), we shall consider the following space

$$
\mathscr{F}_{T}\left(\mathbb{R}, \mathbb{R}^{n}\right)=\left\{x \in \mathscr{F}\left(\mathbb{R}, \mathbb{R}^{n}\right): x \text { is } T \text {-periodic }\right\},
$$


where

$\mathscr{F}\left(\mathbb{R}, \mathbb{R}^{n}\right)=\left\{x: \mathbb{R} \rightarrow \mathbb{R}^{n}: x \in C\left(\left(t_{j}, t_{j+1}\right], \mathbb{R}^{n}\right)\right.$ and the lateral limit $x\left(t_{j}^{+}\right)$exists for all $\left.j \in \mathbb{Z}\right\}$

The space $\mathscr{F}_{T}\left(\mathbb{R}, \mathbb{R}^{n}\right)$ is complete with the norm $|f|_{T}=\sup _{t \in[0, T]}\|f(t)\|$, where $\|\cdot\|$ is a norm in $\mathbb{R}^{n}$, thus $\mathscr{F}_{T}\left(\mathbb{R}, \mathbb{R}^{n}\right)$ is a Banach space.

The idea to obtain periodic solutions for the impulsive NFDE (6.1) is to establish a correspondence between the impulsive NFDE (6.1) and a non-impulsive neutral differential equation. Thus, we can use the results presented in Chapter 5 to find periodic solutions for the corresponding non-impulsive neutral differential equation, and, via the correspondence, we conclude the existence of periodic solutions for the impulsive NFDE (6.1).

Next, we present the general conditions that we shall consider in this section.

(I1) there are $T>0$ and $q \in \mathbb{Z}_{+}$such that $0<t_{0}<t_{1}<\ldots<t_{q-1}<T$ and $t_{q+k}=t_{k}+T$, for all $k \in \mathbb{Z}$;

(I2) for all $k \in \mathbb{Z}, b_{k}$ is a real number larger than -1 and $b_{q+k}=b_{k}$. Furthermore,

$$
\prod_{j=0}^{q-1}\left(1+b_{j}\right)=1
$$

Associated to the sequence $\left\{b_{k}\right\}_{k \in \mathbb{Z}}$, we consider the following auxiliary function

$$
\zeta(t)= \begin{cases}1, & \text { if } t \in\left(k T, t_{0}+k T\right], \\ \prod_{k T<t_{j}<t}\left(1+b_{j}\right), & \text { if } t \in\left(t_{0}+k T,(k+1) T\right], k \in \mathbb{Z} .\end{cases}
$$

(A1) $B, g: \mathbb{R} \times \mathscr{F}\left(\mathbb{R}, \mathbb{R}^{n}\right) \rightarrow \mathbb{R}^{n}$ are $T$-periodic functions with respect to the first variable;

(A2) for each $x \in \mathscr{F}_{T}\left(\mathbb{R}, \mathbb{R}^{n}\right)$ the mapping $t \mapsto g\left(t, x_{t}\right)$ is locally Perron integrable over $\mathbb{R}$ and the mapping $t \mapsto B\left(t, x_{t}\right)$ belongs to the space $\mathscr{F}_{T}\left(\mathbb{R}, \mathbb{R}^{n}\right)$ and is differentiable almost everywhere on $\mathbb{R}$ whenever $x$ is differentiable almost everywhere on $\mathbb{R}$;

(A3) given $\mu>0$, there exist a bounded function $\eta_{\mu}: \mathbb{R} \rightarrow \mathbb{R}$ and a constant $0<\theta<\frac{1}{4 q+2}$ such that $\sup _{t \in[0, T]}\left|\eta_{\mu}(t)\right| \leq \theta \frac{d}{D}$ and

$$
\left\|B\left(t, x_{t}\right)-B\left(t, y_{t}\right)\right\| \leq\left|\eta_{\mu}(t)\right||x-y|_{T},
$$

for all $t \in[0, T]$ and $x, y \in \mathscr{F}_{T}\left(\mathbb{R}, \mathbb{R}^{n}\right)$ with $|x|_{T} \leq \mu$ and $|y|_{T} \leq \mu$, where $d=\min _{t \in[0, T]} \zeta(t)$ and $D=\max _{t \in[0, T]} \zeta(t)$; 
(A4) given a non-negative function $m: \mathbb{R} \rightarrow \mathbb{R}_{+}$and $\mu>0$, there is a non-negative locally Perron integrable function $N_{\mu}: \mathbb{R} \rightarrow \mathbb{R}_{+}$such that, for all $s_{1}, s_{2} \in[0, T], s_{1} \leq s_{2}$,

$$
\left\|\int_{s_{1}}^{s_{2}} m(t) g\left(t, x_{t}\right) d t\right\| \leq \int_{s_{1}}^{s_{2}} m(t) N_{\mu}(t) d t
$$

whenever $x \in \mathscr{F}_{T}\left(\mathbb{R}, \mathbb{R}^{n}\right)$ and $|x|_{T} \leq \mu$;

(A5) given a non-negative function $\ell: \mathbb{R} \rightarrow \mathbb{R}_{+}$and $\mu>0$, there is a non-negative locally Perron integrable function $H_{\mu}: \mathbb{R} \rightarrow \mathbb{R}_{+}$such that, for all $s_{1}, s_{2} \in[0, T], s_{1} \leq s_{2}$,

$$
\left\|\int_{s_{1}}^{s_{2}} \ell(t)\left[g\left(t, x_{t}\right)-g\left(t, y_{t}\right)\right] d t\right\| \leq \int_{s_{1}}^{s_{2}} \ell(t) H_{\mu}(t)|x-y|_{T} d t
$$

for all $x, y \in \mathscr{F}_{T}\left(\mathbb{R}, \mathbb{R}^{n}\right)$ such that $|x|_{T} \leq \mu$ and $|y|_{T} \leq \mu$.

Motivated by the techniques developed in (LI; KOU; DUAN, 2009), (YAN; ZHAO, 1998) and (ZHAO; FENG; DING, 2015), we introduce the following non-impulsive NFDE

$$
\frac{d}{d t}\left(u(t)-\frac{B\left(t, \zeta_{t} u_{t}\right)}{\zeta(t)}\right)=\frac{g\left(t, \zeta_{t} u_{t}\right)}{\zeta(t)}
$$

almost everywhere in $\mathbb{R}$, where $\zeta$ is given in (6.2) and $\zeta_{t}(\theta)=\zeta(t+\theta)$ for all $t, \theta \in \mathbb{R}$.

Remark 6.1.2. By hypothesis (I1), the impulsive moments of the interval $(k T,(k+1) T]$ are of type $t_{k q+j}=t_{j}+k T$, for all $j=0,1, \ldots, q-1$.

Remark 6.1.3. According to hypotheses (I1) and (I2), if $t \in\left(t_{0}+k T,(k+1) T\right]$ then $t+T \in$ $\left(t_{0}+(k+1) T,(k+2) T\right]$ and

$$
\begin{aligned}
\zeta(t+T) & =\prod_{(k+1) T<t_{(k+1) q+j}<t+T}\left(1+b_{(k+1) q+j}\right) \\
& =\prod_{(k+1) T<t_{j}+(k+1) T<t+T}\left(1+b_{q+(k q+j)}\right) \\
& =\prod_{k T<t_{j}+k T<t}\left(1+b_{k q+j}\right) \\
& =\prod_{k T<t_{k q+j}<t}\left(1+b_{k q+j}\right)=\zeta(t) .
\end{aligned}
$$

Since $\zeta(t)=1$ for all $t \in\left(k T, t_{0}+k T\right]$, we conclude that the function $\zeta$ is $T$-periodic. Moreover, $\zeta$ is continuous at every point $k T, k \in \mathbb{Z}$. In fact, note that

$$
\zeta\left(k T^{+}\right)=\lim _{t \rightarrow(k T)^{+}} \zeta(t)=\lim _{t \rightarrow(k T)^{+}} 1=1=\zeta(k T)
$$

and

$$
\zeta\left(k T^{-}\right)=\lim _{t \rightarrow(k T)^{-}} \zeta(t)=\lim _{t \rightarrow(k T)^{-}} \prod_{(k-1) T<t_{j}<t}\left(1+b_{j}\right)=\prod_{(k-1) T<t_{j}<k T}\left(1+b_{j}\right)=\zeta(k T),
$$

since $\zeta(k T)=\prod_{(k-1) T<t_{j}<k T}\left(1+b_{j}\right)=\prod_{j=0}^{q-1}\left(1+b_{j}\right)=1$. Therefore, $\zeta\left(k T^{-}\right)=\zeta(k T)=\zeta\left(k T^{+}\right)$, for all $k \in \mathbb{Z}$. 
Remark 6.1.4. By condition (A2), $\Lambda_{B}^{[0, T)}=\left\{t_{0}, t_{1}, \ldots, t_{q-1}\right\}$. Recall that $\Lambda_{B}^{[0, T)}$ denotes the set of all discontinuity points of the map $[0, T) \ni t \mapsto B(t, \cdot)$ on the interval $[0, T)$.

The concept of a solution of the non-impulsive NFDE (6.3) is presented in the next definition.

Definition 6.1.5. A function $u: \mathbb{R} \rightarrow \mathbb{R}^{n}$ is said to be a solution of the non-impulsive NFDE (6.3), if $u \in C\left(\mathbb{R}, \mathbb{R}^{n}\right)$ and $u$ satisfies (6.3) almost everywhere in $\mathbb{R}$.

Lemma 6.1.6 gives us a correspondence between the solutions of the impulsive NFDE (6.1) and the solutions of the non-impulsive NFDE (6.3).

Lemma 6.1.6. If $u: \mathbb{R} \rightarrow \mathbb{R}^{n}$ is a solution of the NFDE (6.3), then $x: \mathbb{R} \rightarrow \mathbb{R}^{n}$ given by

$$
x(t)=\zeta(t) u(t), \quad \text { for all } t \in \mathbb{R},
$$

is a solution of the impulsive NFDE (6.1). Conversely, if $x: \mathbb{R} \rightarrow \mathbb{R}^{n}$ is a solution of the impulsive $\operatorname{NFDE}(6.1)$, then the function $u: \mathbb{R} \rightarrow \mathbb{R}^{n}$ given by

$$
u(t)=\frac{x(t)}{\zeta(t)}, \quad \text { for all } t \in \mathbb{R},
$$

is a solution of the NFDE (6.3).

Proof. Let $x(t)=\zeta(t) u(t)$, for all $t \in \mathbb{R}$, where $u: \mathbb{R} \rightarrow \mathbb{R}^{n}$ is a solution of the NFDE (6.3). By Definition 6.1.5, $u \in C\left(\mathbb{R}, \mathbb{R}^{n}\right)$ and $u$ satisfies (6.3) almost everywhere in $\mathbb{R}$. Since $\zeta$ is continuous on each subinterval $\left(t_{j}, t_{j+1}\right], j \in \mathbb{Z}$, we have $x \in C\left(\left(t_{j}, t_{j+1}\right], \mathbb{R}^{n}\right)$ for every $j \in \mathbb{Z}$. Now, for almost every $t \in \mathbb{R} \backslash\left\{t_{j}: j \in \mathbb{Z}\right\}$, we have

$$
\begin{aligned}
\frac{d}{d t}\left(x(t)-B\left(t, x_{t}\right)\right) & =\frac{d}{d t}\left[\zeta(t)\left(u(t)-\frac{B\left(t, \zeta_{t} u_{t}\right)}{\zeta(t)}\right)\right] \\
& =\zeta(t) \frac{d}{d t}\left(u(t)-\frac{B\left(t, \zeta_{t} u_{t}\right)}{\zeta(t)}\right) \\
& =g\left(t, \zeta_{t} u_{t}\right)=g\left(t, x_{t}\right) .
\end{aligned}
$$

Let $t_{j}$ be an arbitrary impulsive time. Then there exists $k \in \mathbb{Z}$ such that $t_{j} \in(k T,(k+1) T)$. Since $u\left(t_{j}^{+}\right)=u\left(t_{j}\right)$ and (I1) holds, we obtain

$$
\begin{aligned}
x\left(t_{j}^{+}\right)-x\left(t_{j}\right) & =\lim _{t \rightarrow t_{j}^{+}} \zeta(t) u(t)-x\left(t_{j}\right)=\lim _{t \rightarrow t_{j}^{+}} \prod_{k T<t_{i}<t}\left(1+b_{i}\right) u(t)-x\left(t_{j}\right) \\
& =\prod_{k T<t_{i} \leq t_{j}}\left(1+b_{i}\right) u\left(t_{j}^{+}\right)-x\left(t_{j}\right)=\frac{x\left(t_{j}\right)}{\zeta\left(t_{j}\right)}\left(1+b_{j}\right) \prod_{k T<t_{i}<t_{j}}\left(1+b_{i}\right)-x\left(t_{j}\right) \\
& =x\left(t_{j}\right)\left(1+b_{j}\right)-x\left(t_{j}\right)=b_{j} x\left(t_{j}\right) .
\end{aligned}
$$

Hence, $x: \mathbb{R} \rightarrow \mathbb{R}^{n}$ is a solution of the impulsive NFDE (6.1). 

NFDE (6.1).

Conversely, let $u(t)=\frac{x(t)}{\zeta(t)}$, for all $t \in \mathbb{R}$, where $x: \mathbb{R} \rightarrow \mathbb{R}^{n}$ is a solution of the impulsive

Since $x$ and $\zeta$ are continuous on each interval $\left(t_{j}, t_{j+1}\right], j \in \mathbb{Z}$, we conclude that $u$ is continuous on each interval $\left(t_{j}, t_{j+1}\right], j \in \mathbb{Z}$. Further, let $t_{j}$ be an impulsive time. Let $k \in \mathbb{Z}$ be such that $t_{j} \in(k T,(k+1) T)$. Then

$$
\begin{aligned}
u\left(t_{j}^{+}\right) & =\lim _{t \rightarrow t_{j}^{+}} u(t)=\lim _{t \rightarrow t_{j}^{+}} \frac{x(t)}{\zeta(t)}=\lim _{t \rightarrow t_{j}^{+}} \prod_{k T<t_{i}<t}\left(1+b_{i}\right)^{-1} x(t) \\
& =\prod_{k T<t_{i} \leq t_{j}}\left(1+b_{i}\right)^{-1} x\left(t_{j}^{+}\right) \\
& =\left(1+b_{j}\right)^{-1} \zeta^{-1}\left(t_{j}\right)\left(1+b_{j}\right) x\left(t_{j}\right) \\
& =\frac{x\left(t_{j}\right)}{\zeta\left(t_{j}\right)}=u\left(t_{j}\right) .
\end{aligned}
$$

Consequently, $u \in C\left(\mathbb{R}, \mathbb{R}^{n}\right)$.

Lastly, we have

$$
\begin{aligned}
\frac{d}{d t}\left(u(t)-\frac{B\left(t, \zeta_{t} u_{t}\right)}{\zeta(t)}\right) & =\frac{d}{d t}\left(\frac{1}{\zeta(t)}\left(x(t)-B\left(t, \zeta_{t} u_{t}\right)\right)\right) \\
& =\frac{1}{\zeta(t)} \frac{d}{d t}\left(x(t)-B\left(t, x_{t}\right)\right) \\
& =\frac{g\left(t, x_{t}\right)}{\zeta(t)}=\frac{g\left(t, \zeta_{t} u_{t}\right)}{\zeta(t)}
\end{aligned}
$$

almost everywhere in $\mathbb{R}$. Therefore, $u: \mathbb{R} \rightarrow \mathbb{R}^{n}$ is a solution of the NFDE (6.3).

Lemma 6.1.7. Assume that conditions (I1)-(I2) and (A1)-(A5) hold. Then, conditions (J1)-(J5) are satisfied with $A(t, \phi)=\frac{B\left(t, \zeta_{t} \phi\right)}{\zeta(t)}$ and $f(t, \phi)=\frac{g\left(t, \zeta_{t} \phi\right)}{\zeta(t)}$ for all $(t, \phi) \in \mathbb{R} \times C\left(\mathbb{R}, \mathbb{R}^{n}\right)$.

Proof. Conditions (J1)-(J2) follow from conditions (A1)-(A2) and by the periodicity of $\zeta$.

Let $\mu>0$ be given and take $u, v \in C_{T}\left(\mathbb{R}, \mathbb{R}^{n}\right)$ such that $|u|_{T} \leq \mu$ and $|v|_{T} \leq \mu$. The condition (A3) ensures that, there exist a function $\eta_{D \mu}: \mathbb{R} \rightarrow \mathbb{R}$ and $0<\theta<\frac{1}{4 q+2}$, with

$$
\sup _{t \in[0, T]}\left|\eta_{D \mu}(t)\right| \leq \theta \frac{d}{D}
$$

such that

$$
\left\|B\left(t, \zeta_{t} u_{t}\right)-B\left(t, \zeta_{t} v_{t}\right)\right\| \leq\left|\eta_{D \mu}(t)\right||\zeta u-\zeta v|_{T}
$$

for all $t \in[0, T]$, since $|\zeta u|_{T} \leq D \mu$ and $|\zeta v|_{T} \leq D \mu$. 
Now, define the function $\gamma_{\mu}: \mathbb{R}_{+} \rightarrow \mathbb{R}+$ by $\gamma_{\mu}(s)=\theta s$, for all $s \in[0, \infty)$. Then, we have

$$
\begin{aligned}
\left\|A\left(t, u_{t}\right)-A\left(t, v_{t}\right)\right\| & =\frac{1}{\zeta(t)}\left\|B\left(t, \zeta_{t} u_{t}\right)-B\left(t, \zeta_{t} v_{t}\right)\right\| \\
& \leq \frac{1}{d}\left|\eta_{D \mu}(t)\right||\zeta u-\zeta v|_{T} \\
& \leq \frac{D}{d}\left|\eta_{D \mu}(t)\right||u-v|_{T} \\
& \leq \theta \frac{D}{d} \frac{d}{D}|u-v|_{T} \\
& =\theta|u-v|_{T}=\gamma_{\mu}\left(|u-v|_{T}\right) .
\end{aligned}
$$

Hence, (J3) holds.

To verify condition (J4), let $\mu>0$ be given and $u \in C_{T}\left(\mathbb{R}, \mathbb{R}^{n}\right)$ be such that $|u|_{T} \leq \mu$. Considering the function $m(t)=\frac{1}{\zeta(t)}$, it follows from condition (A4) the existence of a nonnegative locally Perron integrable function $N_{D \mu}: \mathbb{R} \rightarrow \mathbb{R}_{+}$such that

$$
\left\|\int_{s_{1}}^{s_{2}} f\left(t, u_{t}\right) d t\right\|=\left\|\int_{s_{1}}^{s_{2}} \frac{1}{\zeta(t)}\left[g\left(t, \zeta_{t} u_{t}\right)\right] d t\right\| \leq \int_{s_{1}}^{s_{2}} \frac{1}{\zeta(t)} N_{D \mu}(t) d t
$$

for all $s_{1}, s_{2} \in[0, T], s_{1} \leq s_{2}$, since $|\zeta u|_{T} \leq D \mu$. Therefore, if we define a non-negative locally Perron integrable function $F_{\mu}: \mathbb{R} \rightarrow \mathbb{R}_{+}$by $F_{\mu}(t)=\frac{1}{\zeta(t)} N_{D \mu}(t)$, for all $t \in \mathbb{R}$, then we conclude that condition (J4) holds.

At last, let us show that condition (J5) holds. For this, let $\mu>0$ and $u, v \in C_{T}\left(\mathbb{R}, \mathbb{R}^{n}\right)$ be given such that $|u|_{T} \leq \mu$ and $|v|_{T} \leq \mu$. Taking $\ell(t)=\frac{1}{\zeta(t)}$, it follows by condition (A5) that there is a non-negative locally Perron integrable function $H_{D \mu}: \mathbb{R} \rightarrow \mathbb{R}_{+}$such that

$$
\begin{aligned}
\left\|\int_{s_{1}}^{s_{2}}\left[f\left(t, u_{t}\right)-f\left(t, v_{t}\right)\right] d t\right\| & =\left\|\int_{s_{1}}^{s_{2}} \frac{1}{\zeta(t)}\left[g\left(t, \zeta_{t} u_{t}\right)-g\left(t, \zeta_{t} v_{t}\right)\right] d t\right\| \\
& \leq \int_{s_{1}}^{s_{2}} \frac{1}{\zeta(t)} H_{D \mu}(t)|\zeta u-\zeta v|_{T} d t \\
& \leq \int_{s_{1}}^{s_{2}} \frac{1}{\zeta(t)} H_{D \mu}(t) D|u-v|_{T} d t,
\end{aligned}
$$

for all $s_{1}, s_{2} \in[0, T]$ with $s_{1} \leq s_{2}$, as $|\zeta u|_{T} \leq D \mu$ and $|\zeta v|_{T} \leq D \mu$. Therefore, if we define a non-negative locally Perron integrable function $L_{\mu}: \mathbb{R} \rightarrow \mathbb{R}_{+}$by $L_{\mu}(t)=\frac{D}{\zeta(t)} H_{D \mu}(t)$, for all $t \in \mathbb{R}$, then condition (J5) is satisfied.

The next result, namely Theorem 6.1.8, guarantees the existence of a periodic solution for the impulsive NFDE (6.1) in $U_{\beta}=\left\{u \in \mathscr{F}_{T}\left(\mathbb{R}, \mathbb{R}^{n}\right):|u|_{T} \leq \beta\right\}$, for an adequate $\beta>0$.

Theorem 6.1.8. Assume that conditions (I1)-(I2) and (A1)-(A5) hold. If $\beta>0$ is such that

$$
\sup _{t \in[0, T]} \frac{\|B(t, 0)\|}{|\zeta(t)|}+\sum_{0<t_{k}<T}\left(\frac{\left\|B\left(t_{k}, 0\right)\right\|}{\left|\zeta\left(t_{k}\right)\right|}+\frac{\left\|B\left(t_{k}^{+}, 0\right)\right\|}{\left|\zeta\left(t_{k}^{+}\right)\right|}\right)+\int_{0}^{T} \frac{N_{D \beta}(s)}{\zeta(s)} d s \leq \frac{\beta}{2},
$$

then the impulsive NFDE (6.1) has at least one $T$-periodic solution in $U_{\beta}=\left\{u \in \mathscr{F}_{T}\left(\mathbb{R}, \mathbb{R}^{n}\right):|u|_{T} \leq\right.$ $\beta\}$. 
Proof. In fact, by Lemma 6.1.7, the functions

$$
f(t, \phi)=\frac{g\left(t, \zeta_{t} \phi\right)}{\zeta(t)} \quad \text { and } \quad A(t, \phi)=\frac{B\left(t, \zeta_{t} \phi\right)}{\zeta(t)}, \quad(t, \phi) \in \mathbb{R} \times C\left(\mathbb{R}, \mathbb{R}^{n}\right)
$$

satisfy conditions (J1)-(J5). And yet, by the proof of Lemma 6.1.7, the function $f$ satisfies condition (J4) with $F_{\mu}(t)=\frac{1}{\zeta(t)} N_{D \mu}(t)$, for all $t \in \mathbb{R}$. Thus, condition (6.4) implies condition (5.10). Consequently, by Theorem 5.1.9, the non-impulsive NFDE (6.3) admits at least one $T$-periodic solution in $\left\{u \in C_{T}\left(\mathbb{R}, \mathbb{R}^{n}\right):|u|_{T} \leq \beta\right\}$. Therefore, by Lemma 6.1.6, the impulsive $\operatorname{NFDE}$ (6.1) has at least one $T$-periodic solution in $U_{\beta}=\left\{u \in \mathscr{F}_{T}\left(\mathbb{R}, \mathbb{R}^{n}\right):|u|_{T} \leq \beta\right\}$.

Theorem 6.1.9 below provides sufficient conditions for uniqueness of periodic solutions of the impulsive NFDE (6.1).

Theorem 6.1.9. Under the conditions of Theorem 6.1.8, assume that

$$
\int_{0}^{T} \frac{D}{\zeta(s)} H_{D \beta}(s) d s<\frac{1}{2}
$$

Then the impulsive NFDE (6.1) has a unique $T$-periodic solution in $U_{\beta}$.

Proof. By Lemma 6.1.7, the functions

$$
f(t, \phi)=\frac{g\left(t, \zeta_{t} \phi\right)}{\zeta(t)} \quad \text { and } \quad A(t, \phi)=\frac{B\left(t, \zeta_{t} \phi\right)}{\zeta(t)}, \quad(t, \phi) \in \mathbb{R} \times C\left(\mathbb{R}, \mathbb{R}^{n}\right),
$$

satisfy conditions (J1)-(J5). By the proof of Theorem 6.1.8, we have condition (5.10) is satisfied. By the proof of Lemma 6.1.7, the function $f$ satisfies condition (J5) with $L_{\mu}(t)=\frac{D}{\zeta(t)} H_{D \mu}(t)$, for all $t \in \mathbb{R}$. Thus, condition (6.5) implies condition (5.14). Therefore, by Theorem 5.1.10 and Lemma 6.1.6, we conclude that the impulsive NFDE (6.1) has a unique $T$-periodic solution in $U_{\beta}=\left\{u \in \mathscr{F}_{T}\left(\mathbb{R}, \mathbb{R}^{n}\right):|u|_{T} \leq \beta\right\}$.

\subsection{Examples}

This section concerns some applications. In Example 6.2.1, we exhibit an impulsive neutral differential equation with delay of the Lasota-Wazewska type. Such kind of equations were studied in (STAMOVA; STAMOV, 2016) for instance.

Example 6.2.1. Consider the impulsive Lasota-Wazewska equation type:

$$
\left\{\begin{array}{l}
\frac{d}{d t}(x(t)-C(t) x(t-r))=-A(t) x(t)+\sum_{j=1}^{n} d_{j}(t) e^{-\gamma_{j}(t) x_{j}(t-\tau)}, t \neq t_{k}, k \in \mathbb{Z}, \\
x\left(t_{k}^{+}\right)-x\left(t_{k}\right)=\frac{2}{\left(4+(-1)^{k+1}+1\right)} \cos (k \pi) x\left(t_{k}\right), \quad k \in \mathbb{Z},
\end{array}\right.
$$

where $C(t)=\operatorname{diag}\left(c_{1}(t), \ldots, c_{n}(t)\right), A(t)=\operatorname{diag}\left(a_{1}(t), \ldots, a_{n}(t)\right), c_{j}, a_{j} \in \mathscr{F}_{T}\left(\mathbb{R}_{1}, \mathbb{R}_{+}\right), d_{j} \in$ $\mathscr{F}_{T}\left(\mathbb{R}, \mathbb{R}^{n}\right), \gamma_{j} \in \mathscr{F}_{T}(\mathbb{R},(0, \infty))$ for all $j=1, \ldots, n$, and $r, \tau \geq 0$. Furthermore, we assume 
that $0<t_{0}<t_{1}<\ldots<t_{q-1}<T$ and $t_{k+q}=t_{k}+T$, for all $k \in \mathbb{Z}$, with $q$ even. Since $b_{k}=$ $\frac{2}{\left(4+(-1)^{k+1}+1\right)} \cos (k \pi)$ and $q$ is even, we have

$$
b_{k+q}=\frac{2}{\left(4+(-1)^{k+q+1}+1\right)} \cos ((k+q) \pi)=\frac{2}{\left(4+(-1)^{k+1}+1\right)} \cos (k \pi)=b_{k}
$$

for all $k \in \mathbb{Z}$. Moreover, if $k \in \mathbb{Z}$ is an even number then

$$
b_{k}=\frac{2}{\left(4+(-1)^{k+1}+1\right)} \cos (k \pi)=\frac{1}{2}
$$

and, if $k \in \mathbb{Z}$ is an odd number then

$$
b_{k}=\frac{2}{\left(4+(-1)^{k+1}+1\right)} \cos (k \pi)=\frac{2}{\left(4+(-1)^{k+1}+1\right)}=-\frac{1}{3} .
$$

Therefore,

$$
\prod_{j=0}^{q-1}\left(1+b_{j}\right)=\left(\frac{3}{2}\right)^{q / 2}\left(\frac{2}{3}\right)^{q / 2}=1 .
$$

Hence, conditions (I1)-(I2) are valid.

The function $\zeta(t)$ is given by

$$
\zeta(t)= \begin{cases}1, & \text { if } t \in\left(k T, t_{0}+k T\right], \\ \prod_{k T<t_{j}<t}\left(1+\frac{2}{\left(4+(-1)^{j+1}+1\right)} \cos (j \pi)\right), & \text { if } t \in\left(t_{0}+k T,(k+1) T\right], k \in \mathbb{Z} .\end{cases}
$$

Note that $d=\min _{t \in[0, T]} \zeta(t)=\min \left\{1, \frac{3}{2}\right\}=1$ and $D=\max _{t \in[0, T]} \zeta(t)=\max \left\{1, \frac{3}{2}\right\}=\frac{3}{2}$.

In addition, assume that the following conditions hold:

(a) there is a constant $0<\omega<\frac{2}{6 q+3}$ such that $\sup _{t \in[0, T]}\|C(t)\| \leq \frac{\omega}{2}$;

(b) the mapping $C: \mathbb{R} \rightarrow M_{n}(\mathbb{R})$ is differentiable almost everywhere on $\mathbb{R}$;

(c) given a non-negative function $m: \mathbb{R} \rightarrow \mathbb{R}_{+}$, there is a non-negative Perron integrable functions $M: \mathbb{R} \rightarrow \mathbb{R}_{+}$such that

$$
\left\|\int_{s_{1}}^{s_{2}} m(t) A(t)[\phi(t)-\psi(t)] d t\right\| \leq \int_{s_{1}}^{s_{2}} m(t) M(t)|\phi-\psi|_{T} d t
$$

whenever $s_{1}, s_{2} \in[0, T], s_{1} \leq s_{2}$, and $\phi, \psi \in \mathscr{F}_{T}\left(\mathbb{R}, \mathbb{R}^{n}\right)$;

(d) $\int_{0}^{T} M(t) d t \leq \frac{1}{6}$;

(e) there exists $\alpha>0$ such that $\sup _{t \in[0, T]} \sum_{j=1}^{n}\left\|d_{j}(s)\right\| e^{\gamma_{j}(s) D \alpha} \leq \frac{\alpha}{4 T}$. 
Let $B, g: \mathbb{R} \times \mathscr{F}\left(\mathbb{R}, \mathbb{R}^{n}\right) \rightarrow \mathbb{R}$ be defined by

$$
B(t, \phi)=C(t) \phi(-r) \quad \text { and } \quad g(t, \phi)=-A(t) \phi(0)+\sum_{j=1}^{n} d_{j}(t) e^{-\gamma_{j}(t) \phi_{j}(-\tau)} .
$$

Since $c_{j}, a_{j} \in \mathscr{F}_{T}\left(\mathbb{R}, \mathbb{R}_{+}\right), d_{j} \in \mathscr{F}_{T}\left(\mathbb{R}, \mathbb{R}^{n}\right)$ and $\gamma_{j} \in \mathscr{F}_{T}(\mathbb{R},(0, \infty))$ for all $j=1, \ldots, n$, condition (A1) holds. Hypothesis (A2) follows from condition (b) and from the fact that the mapping $t \mapsto-A(t) x(t)+\sum_{j=1}^{n} d_{j}(t) e^{-\gamma_{j}(t) x_{j}(t-\tau)}$ is locally Perron integrable over $\mathbb{R}$ whenever $x \in \mathscr{F}_{T}\left(\mathbb{R}, \mathbb{R}^{n}\right)$.

Let us verify that condition (A3) holds. Let $\mu>0$ and $x, y \in \mathscr{F}_{T}\left(\mathbb{R}, \mathbb{R}^{n}\right)$ be such that $|x|_{T} \leq \mu$ and $|y|_{T} \leq \mu$. Setting $\eta_{\mu}(t)=\|C(t)\|, t \in \mathbb{R}$, we get

$$
\left\|B\left(t, x_{t}\right)-B\left(t, y_{t}\right)\right\|=\|C(t)\|\|x(t-r)-y(t-r)\| \leq\left|\eta_{\mu}(t) \| x-y\right|_{T},
$$

for all $t \in[0, T]$. Taking $\theta=\frac{3}{4} \omega$, it follows by condition (a) that $\theta \in\left(0, \frac{1}{4 q+2}\right)$ and

$$
\sup _{t \in[0, T]}\left|\eta_{\mu}(t)\right| \leq \frac{\omega}{2}=\frac{\theta d}{D} .
$$

Let us verify that condition (A4) hold. Given $\mu>0$, let $x \in \mathscr{F}_{T}\left(\mathbb{R}, \mathbb{R}^{n}\right)$ be such that $|x|_{T} \leq \mu$. Given a non-negative function $m: \mathbb{R} \rightarrow \mathbb{R}_{+}$and $s_{1}, s_{2} \in[0, T], s_{1} \leq s_{2}$, it follows by (c) that

$$
\begin{aligned}
\left\|\int_{s_{1}}^{s_{2}} m(t) g\left(t, x_{t}\right) d t\right\| & \leq\left\|\int_{s_{1}}^{s_{2}} m(t) A(t) x(t) d t\right\|+\left\|\sum_{j=1}^{n} \int_{s_{1}}^{s_{2}} m(t) d_{j}(t) e^{-\gamma_{j}(t) x_{j}(t-\tau)} d t\right\| \\
& \leq \int_{s_{1}}^{s_{2}} m(t) M(t)|x|_{T} d t+\sum_{j=1}^{n} \int_{s_{1}}^{s_{2}} m(t)\left\|d_{j}(t)\right\| e^{-\gamma_{j}(t) x_{j}(t-\tau)} d t \\
& \leq \int_{s_{1}}^{s_{2}} m(t) M(t) \mu d t+\sum_{j=1}^{n} \int_{s_{1}}^{s_{2}} m(t)\left\|d_{j}(t)\right\| e^{\gamma_{j}(t) \mu} d t \\
& \leq \int_{s_{1}}^{s_{2}} m(t) N_{\mu}(t) d t,
\end{aligned}
$$

where $N_{\mu}(t)=M(t) \mu+\sum_{j=1}^{n}\left\|d_{j}(t)\right\| e^{\gamma_{j}(t) \mu}$ for all $t \in \mathbb{R}$.

In order to verify condition (A5), let $\ell: \mathbb{R} \rightarrow \mathbb{R}_{+}$be a non-negative function and $\mu>0$. Also, let $s_{1}, s_{2} \in[0, T]$ with $s_{1} \leq s_{2}$ and take $x, y \in \mathscr{F}_{T}\left(\mathbb{R}, \mathbb{R}^{n}\right)$ such that $|x|_{T} \leq \mu$ and $|y|_{T} \leq \mu$. As the exponential function is locally Lipschitzian, there exists $K_{\mu}>0$ such that

$$
\left|\sum_{j=1}^{n} d_{j i}(t) e^{-\gamma_{j}(t) x_{j}(t-r)}-\sum_{j=1}^{n} d_{j i}(t) e^{-\gamma_{j}(t) y_{j}(t-r)}\right| \leq K_{\mu}|x-y|_{T},
$$

for all $i=1, \ldots, n$ and for all $t \in[0, T]$, where $d_{j}(t)=\left(d_{j 1}(t), \ldots, d_{j n}(t)\right)$.

There exists $\tilde{\eta}>0$ such that $\|\cdot\| \leq \tilde{\eta}\|\cdot\|_{1}$, where $\|M\|_{1}=\max _{1 \leq i \leq n} \sum_{j=1}^{n}\left|m_{i j}(t)\right|$, $M \in \mathbb{M}_{n \times m}(\mathbb{R})$. Then,

$$
\left\|\sum_{j=1}^{n} \int_{s_{1}}^{s_{2}} \ell(t) d_{j}(t)\left[e^{-\gamma_{j}(t) x_{j}(t-r)}-e^{-\gamma_{j}(t) y_{j}(t-r)}\right] d t\right\| \leq \int_{s_{1}}^{s_{2}} \tilde{\eta} K_{\mu} \ell(t)|x-y|_{T} d t .
$$


Thus, we conclude that

$$
\left\|\int_{s_{1}}^{s_{2}} \ell(t)\left[g\left(t, x_{t}\right)-g\left(t, y_{t}\right)\right] d t\right\| \leq \int_{s_{1}}^{s_{2}} \ell(t)\left[M(t)+\tilde{\eta} K_{\mu}\right]|x-y|_{T} d t,
$$

for all $x, y \in \mathscr{F}_{T}\left(\mathbb{R}, \mathbb{R}^{n}\right)$ such that $|x|_{T} \leq \mu$ and $|y|_{T} \leq \mu$, and for all $s_{1}, s_{2} \in[0, T]$ with $s_{1} \leq s_{2}$. Hence, condition (A5) holds by setting $H_{\mu}: \mathbb{R} \rightarrow \mathbb{R}_{+}$by $H_{\mu}(t)=M(t)+\tilde{\eta} K_{\mu}, t \in \mathbb{R}$.

Now, let us prove that condition (6.4) of Theorem 6.1 .8 holds. Take $\beta>0$ satisfying condition (e). Since $\frac{B(t, 0)}{\zeta(t)}=0$ for all $t \in \mathbb{R}, D=\frac{3}{2}$ and $\frac{1}{\zeta(t)} \leq 1$, for all $t \in[0, T]$, we get

$$
\begin{aligned}
& \sup _{t \in[0, T]} \frac{\|B(t, 0)\|}{|\zeta(t)|}+\sum_{0<t_{k}<T}\left(\frac{\left\|B\left(t_{k}, 0\right)\right\|}{\left|\zeta\left(t_{k}\right)\right|}+\frac{\left\|B\left(t_{k}^{+}, 0\right)\right\|}{\left|\zeta\left(t_{k}^{+}\right)\right|}\right)+\int_{0}^{T} \frac{N_{D \beta}(s)}{\zeta(s)} d s \\
& \leq \int_{0}^{T}\left(\frac{M(s) D \beta}{\zeta(s)}+\frac{1}{\zeta(s)} \sum_{j=1}^{n}\left\|d_{j}(s)\right\| e^{\gamma_{j}(s) D \beta}\right) d s \\
& \leq \frac{3 \beta}{2} \int_{0}^{T} M(s) d s+T \sup _{t \in[0, T]} \sum_{j=1}^{n}\left\|d_{j}(s)\right\| e^{\gamma_{j}(s) D \beta} \\
& \leq \frac{3 \beta}{2} \frac{1}{6}+T \frac{\beta}{4 T}=\frac{\beta}{4}+\frac{\beta}{4}=\frac{\beta}{2},
\end{aligned}
$$

as condition (d) holds. Therefore, by Theorem 6.1.8, there exists at least one $T$-periodic solution of equation (6.6) in $U_{\beta}=\left\{u \in \mathscr{F}_{T}\left(\mathbb{R}, \mathbb{R}^{n}\right):\|u\|_{T} \leq \beta\right\}$.

In Example 6.2.2, we study the existence of periodic solutions for a neural-type impulsive equation. These kind of equations are studied by several authors, among them we can mention (AOUITI; M'HAMDI; CHÉRIF, 2017) and (STAMOVA; STAMOV, 2016).

Example 6.2.2. Consider the impulsive neural networks differential equation with infinity delay model:

$$
\left\{\begin{array}{l}
\frac{d}{d t}(x(t)-P(t) x(t-r(t)))=-C(t) x(t-\sigma(t))+A(t) h(x(t-\tau(t))) \\
+\int_{-\infty}^{t} K(t-s) g(x(s)) d s+J(t), \quad t \neq t_{k}, k \in \mathbb{Z}, \\
x\left(t_{k}^{+}\right)-x\left(t_{k}\right)=b_{k} x\left(t_{k}\right), \quad k \in \mathbb{Z},
\end{array}\right.
$$

where $x(t)=\left(x_{1}(t), \ldots, x_{n}(t)\right)^{T}$ is the neuron state vector of the neural networks, $\dot{x}$ denotes the time derivative of the neuron state, $C(t)=\operatorname{diag}\left(c_{1}(t), \ldots, c_{n}(t)\right)$ is a diagonal matrix, $A(t)=$ $\left(a_{i j}(t)\right)_{n \times n}$ and $P(t)=\left(p_{i j}(t)\right)_{n \times n}$ are the interconnection matrices representing the weight coefficients of the neurons, $J(t)=\left(J_{1}(t), \ldots, J_{n}(t)\right)$ is an external input, $h(x(t))$ and $g(x(t))$ are the neuron activation functions, $K(t)=\operatorname{diag}\left(K_{1}(t), \ldots, K_{n}(t)\right)$ is the delay kernel and $b \in \mathbb{R}$. Moreover, for a given matrix $D=\left(d_{i j}(t)\right)_{n \times n}$, we consider the norm $\|D\|=\max _{1 \leq i \leq n} \sum_{j=1}^{n}\left|d_{i j}(t)\right|$.

We shall assume that the following conditions are satisfied:

(i) $b_{k} \in(-1, \infty)$, there are $q \in \mathbb{Z}_{+}$and $T>0$ such that $0<t_{0}<\ldots<t_{q-1}<T, t_{q+k}=t_{k}+T$ and $b_{q+k}=b_{k}$, for all $k \in \mathbb{Z}$. Further, $\prod_{j=0}^{q-1}\left(1+b_{j}\right)=1$. 
In this way, conditions (I1)-(I2) are satisfied.

Now, consider the function $\zeta: \mathbb{R} \rightarrow(0, \infty)$ given by

$$
\zeta(t)= \begin{cases}1, & \text { if } t \in\left(k T, t_{0}+k T\right], \\ \prod_{k T<t_{j}<t}\left(1+b_{j}\right), & \text { if } t \in\left(t_{0}+k T,(k+1) T\right], k \in \mathbb{Z} .\end{cases}
$$

Let $d=\min _{t \in[0, T]} \zeta(t)$ and $D=\max _{t \in[0, T]} \zeta(t)$. Furthermore, we shall consider system (6.7) under the following conditions:

(a) $r, \sigma, \tau \in \mathscr{F}_{T}(\mathbb{R}, \mathbb{R}), g, h \in C\left(\mathbb{R}^{n}, \mathbb{R}^{n}\right), p_{i j}, a_{i j}, c_{j}, J_{j}, K_{j} \in \mathscr{F}_{T}(\mathbb{R}, \mathbb{R})$ for all $i, j \in\{1, \ldots, n\}$;

(b) there is a constant $0<\theta<\frac{1}{4 q+2}$ such that $\sup _{t \in[0, T]}\|P(t)\| \leq \frac{\theta d}{D}$;

(c) the mapping $P: \mathbb{R} \rightarrow M_{n}(\mathbb{R})$ is differentiable almost everywhere on $\mathbb{R}$ and, for each $x \in \mathscr{F}_{T}\left(\mathbb{R}, \mathbb{R}^{n}\right)$, the Perron integral $\int_{-\infty}^{t} K(t-s) g(x(s)) d s$ exists for every $t \in \mathbb{R}$ and the mapping $t \mapsto \int_{-\infty}^{t} K(t-s) g(x(s)) d s$ is locally Perron integrable over $\mathbb{R}$;

(d) given a non-negative function $m: \mathbb{R} \rightarrow \mathbb{R}_{+}$, there exists a non-negative locally Perron integrable function $Q: \mathbb{R} \rightarrow \mathbb{R}_{+}$such that, for all $s_{1}, s_{2} \in[0, T], s_{1} \leq s_{2}$, we have

$$
\left\|\int_{s_{1}}^{s_{2}} m(t) J(t) d t\right\| \leq \int_{s_{1}}^{s_{2}} m(t) Q(t) d t
$$

(e) given a non-negative function $m: \mathbb{R} \rightarrow \mathbb{R}_{+}$, there are non-negative locally Perron integrable functions $L, V, E_{1}, E_{2}: \mathbb{R} \rightarrow \mathbb{R}_{+}$such that, for all $s_{1}, s_{2} \in[0, T], s_{1} \leq s_{2}$,

$$
\left\|\int_{s_{1}}^{s_{2}} m(t) A(t) h(x(t-\tau(t))) d t\right\| \leq \int_{s_{1}}^{s_{2}} m(t)\left[L(t)|x|_{T}+E_{1}(t)\right] d t
$$

and

$$
\left\|\int_{s_{1}}^{s_{2}} m(t)\left(\int_{-\infty}^{t} K(t-s) g(x(s)) d s\right) d t\right\| \leq \int_{s_{1}}^{s_{2}} m(t)\left[V(t)|x|_{T}+E_{2}(t)\right] d t,
$$

whenever $x \in \mathscr{F}_{T}\left(\mathbb{R}, \mathbb{R}^{n}\right)$;

(f) given a non-negative function $\ell: \mathbb{R} \rightarrow \mathbb{R}_{+}$, there are non-negative locally Perron integrable functions $M, N, O: \mathbb{R} \rightarrow \mathbb{R}_{+}$such that, for all $s_{1}, s_{2} \in[0, T], s_{1} \leq s_{2}$,

$$
\begin{gathered}
\left\|\int_{s_{1}}^{s_{2}} \ell(t) C(t)[x(t-\sigma(t))-y(t-\sigma(t))] d t\right\| \leq \int_{s_{1}}^{s_{2}} \ell(t) M(t)|x-y|_{T} d t \\
\left\|\int_{s_{1}}^{s_{2}} \ell(t) A(t)[h(x(t-\tau(t)))-h(y(t-\tau(t)))] d t\right\| \leq \int_{s_{1}}^{s_{2}} \ell(t) O(t)|x-y|_{T} d t
\end{gathered}
$$

and

$$
\left\|\int_{s_{1}}^{s_{2}} \ell(t)\left(\int_{-\infty}^{t} K(t-s)[g(x(s))-g(y(s))] d s\right) d t\right\| \leq \int_{s_{1}}^{s_{2}} \ell(t) N(t)|x-y|_{T} d t
$$

whenever $x, y \in \mathscr{F}_{T}\left(\mathbb{R}, \mathbb{R}^{n}\right)$; 
(g) $\int_{0}^{T} \frac{D}{d}[M(s)+L(s)+V(s)] d s \leq \frac{1}{4}$

Let $B, g: \mathbb{R} \times \mathscr{F}\left(\mathbb{R}, \mathbb{R}^{n}\right) \rightarrow \mathbb{R}^{n}$ be defined by

$$
\begin{gathered}
B(t, \phi)=P(t) \phi(-r(t)) \quad \text { and } \\
g(t, \phi)=-C(t) \phi(-\sigma(t))+A(t) h(\phi(-r(t)))+\int_{-\infty}^{t} K(t-s) g(\phi(s-t)) d s+J(t),
\end{gathered}
$$

for $t \in \mathbb{R}$. Let us prove that the conditions (A1)-(A5) hold.

Conditions (A1) and (A2) follow from assumptions (a) and (c). Condition (A3) holds, since given $\mu>0$ and $x, y \in \mathscr{F}_{T}\left(\mathbb{R}, \mathbb{R}^{n}\right)$ such that $|x|_{T} \leq \mu$ and $|y|_{T} \leq \mu$, we have

$$
\left\|B\left(t, x_{t}\right)-B\left(t, y_{t}\right)\right\|=\|P(t)\|\|x(t-r(t))-y(t-r(t))\| \leq\left|\eta_{\mu}(t)\right||x-y|_{T},
$$

for all $t \in[0, T]$. Setting $\eta_{\mu}(t)=\|P(t)\|$ for all $t \in \mathbb{R}$, we conclude the desired result as condition (b) holds.

To verify that condition (A4) is valid, let $\mu>0$ be arbitrary and let $x \in \mathscr{F}_{T}\left(\mathbb{R}, \mathbb{R}^{n}\right)$ be such that $|x|_{T} \leq \mu$. Given a non-negative function $m: \mathbb{R} \rightarrow \mathbb{R}_{+}$and $s_{1}, s_{2} \in[0, T], s_{1} \leq s_{2}$, it follows by (d), (e) and (f) (taking $\ell(t)=m(t), t \in \mathbb{R}$ ) that

$$
\begin{aligned}
& \left\|\int_{s_{1}}^{s_{2}} m(t) g\left(t, x_{t}\right) d t\right\| \leq\left\|\int_{s_{1}}^{s_{2}} m(t) A(t) h(x(t-\tau(t))) d t\right\| \\
& +\left\|\int_{s_{1}}^{s_{2}} m(t) C(t) x(t-\sigma(t)) d t\right\|+\left\|\int_{s_{1}}^{s_{2}} m(t)\left(\int_{-\infty}^{t} K(t-s) g(x(s)) d s\right) d t\right\| \\
& +\left\|\int_{s_{1}}^{s_{2}} m(t) J(t) d t\right\| \\
& \leq \int_{s_{1}}^{s_{2}} m(t)\left[L(t)|x|_{T}+E_{1}(t)\right] d t+\int_{s_{1}}^{s_{2}} m(t) M(t)|x|_{T} d t \\
& +\int_{s_{1}}^{s_{2}} m(t)\left[V(t)|x|_{T}+E_{2}(t)\right] d t+\int_{s_{1}}^{s_{2}} m(t) Q(t) d t \\
& \leq \int_{s_{1}}^{s_{2}} m(t)[L(t)+M(t)+V(t)] \mu+m(t)\left[E_{1}(t)+E_{2}(t)+Q(t)\right] d t \\
& =\int_{s_{1}}^{s_{2}} m(t) N_{\mu}(t) d t
\end{aligned}
$$

where $N_{\mu}(t)=[M(t)+L(t)+V(t)] \mu+E_{1}(t)+E_{2}(t)+Q(t)$, for all $t \in \mathbb{R}$.

Furthermore, to verify that condition (A5) holds, let $\ell: \mathbb{R} \rightarrow \mathbb{R}_{+}$be a non-negative function and $\mu>0$. Also, let $s_{1}, s_{2} \in[0, T]$ with $s_{1} \leq s_{2}$ and take $x, y \in \mathscr{F}_{T}\left(\mathbb{R}, \mathbb{R}^{n}\right)$ such that 
$|x|_{T} \leq \mu$ and $|y|_{T} \leq \mu$. By condition (f) we get

$$
\begin{aligned}
& \left\|\int_{s_{1}}^{s_{2}} \ell(t)\left[g\left(t, x_{t}\right)-g\left(t, y_{t}\right)\right] d t\right\| \leq\left\|\int_{s_{1}}^{s_{2}} \ell(t) A(t)[h(x(t-\tau(t)))-h(y(t-\tau(t)))] d t\right\| \\
& +\left\|\int_{s_{1}}^{s_{2}} \ell(t) C(t)[x(t-\sigma(t))-y(t-\sigma(t))] d t\right\| \\
& +\left\|\int_{s_{1}}^{s_{2}} \ell(t)\left(\int_{-\infty}^{t} K(t-s)[g(x(s))-g(y(s))] d s\right) d t\right\| \\
& \leq \int_{s_{1}}^{s_{2}} \ell(t)[O(t)+M(t)+N(t)]|x-y|_{T} d t,
\end{aligned}
$$

where $H_{\mu}(t)=M(t)+O(t)+N(t)$, for all $t \in \mathbb{R}$.

Note that $\frac{\|B(t, 0)\|}{|\zeta(t)|}=0$ for all $t \in \mathbb{R}$. Now, choose a $\beta>0$ such that

$$
\beta \geq \frac{4 T}{d} \sup _{t \in[0, T]}\left[E_{1}(t)+E_{2}(t)+Q(t)\right] .
$$

Then, taking into account the condition $(\mathrm{g})$, we obtain

$$
\begin{aligned}
& \sup _{t \in[0, T]} \frac{\|B(t, 0)\|}{|\zeta(t)|}+\sum_{0<t_{k}<T}\left(\frac{\left\|B\left(t_{k}, 0\right)\right\|}{\left|\zeta\left(t_{k}\right)\right|}+\frac{\left\|B\left(t_{k}^{+}, 0\right)\right\|}{\left|\zeta\left(t_{k}^{+}\right)\right|}\right)+\int_{0}^{T} \frac{N_{D \beta}(s)}{\zeta(s)} d s \\
& \leq \int_{0}^{T} \frac{[M(s)+L(s)+V(s)] D \beta}{d} d s+\int_{0}^{T} \frac{E_{1}(s)+E_{2}(s)+Q(s)}{d} d s \\
& \leq \frac{\beta}{4}+\frac{\beta}{4}=\frac{\beta}{2} .
\end{aligned}
$$

Then, by Theorem 6.1.8, there is a $T$-periodic solution of the impulsive neural networks differential equation (6.7) in $U_{\beta}=\left\{u \in \mathscr{F}_{T}\left(\mathbb{R}, \mathbb{R}^{n}\right):\|u\|_{T} \leq \beta\right\}$. In addition, if

$$
\frac{D T}{d} \sup _{t \in[0, T]}[M(t)+O(t)+N(t)]<\frac{1}{2}
$$

then, by Theorem 6.1.9, the equation (6.7) has a unique $T$-periodic solution in $U_{\beta}$.

In the last example, we present a particular case of Example 6.2.2.

Example 6.2.3. Consider a numerical example of the $2 D$-neural networks with mixed delays (6.7) presented in Example 6.2.2, with $r(t)=\sigma(t)=\tau(t)=\frac{1}{2}|\cos (t)|, T=2 \pi$,

$$
\begin{gathered}
h(t, s)=g(t, s)=\left(\begin{array}{c}
\frac{|t+1|}{2} \\
\frac{|s-1|}{2}
\end{array}\right), \\
K(t)=\left(\begin{array}{cc}
0.01 e^{-t} & 0 \\
0 & 0.01 e^{-t}
\end{array}\right), C(t)=\left(\begin{array}{cc}
0.002+0.001 \cos (t) & 0 \\
0 & 0.002+0.001 \cos (t)
\end{array}\right), \\
J(t)=\left(\begin{array}{c}
-0.2 \sin (t)+0.2 \\
0.4 \cos (t)+0.2
\end{array}\right), P(t)=\left(\begin{array}{cc}
0.02+0.01 \sin (t) & 0.01+0.01 \cos (t) \\
0.02 \cos (t) & 0.02 \cos (t)
\end{array}\right)
\end{gathered}
$$


and

$$
A(t)=\left(\begin{array}{cc}
0.002+0.001 \sin (t) & 0.001+0.001 \cos (t) \\
0.001+0.002 \sin (t) & 0.001 \cos (t)
\end{array}\right)
$$

for all $t, s \in \mathbb{R}$. Assume that $0<t_{0}<t_{1}<T$ with $t_{2+k}=t_{k}+T$, for all $k \in \mathbb{Z}$, and let $b_{0}=-\frac{1}{20}$ and $b_{1}=\frac{1}{19}$, where $b_{2+k}=b_{k}$, for all $k \in \mathbb{Z}$.

Note that $\left(1+b_{0}\right)\left(1+b_{1}\right)=\left(\frac{19}{20}\right)\left(\frac{20}{19}\right)=1$ and

$$
\zeta(t)=\left\{\begin{array}{ll}
1, & \text { if } 2 k \pi<t \leq t_{0}+2 k \pi, \\
\frac{19}{20}, & \text { if } t_{0}+2 k \pi<t \leq t_{1}+2 k \pi, \\
1, & \text { if } t_{1}+2 k \pi<t \leq 2(k+1) \pi,
\end{array} \quad k \in \mathbb{Z} .\right.
$$

Thus, $d=0.95$ and $D=1$.

We have $\|P(t)\| \leq 0.05$ for every $t \in \mathbb{R}$. Setting $\theta=0.09 \in\left(0, \frac{1}{10}\right)$, we get $\|P(t)\|<\theta \frac{d}{D}$ for every $t \in \mathbb{R}$. Thus, condition (b) from Example 6.2.2 holds.

Let us check that conditions (d)-(f) from Example 6.2.2 hold. To verify the validity of condition (d), let $m: \mathbb{R} \rightarrow \mathbb{R}_{+}$be a non-negative function and $s_{1}, s_{2} \in[0,2 \pi]$. Then,

$$
\begin{aligned}
& \left\|\int_{s_{1}}^{s_{2}} m(t) J(t) d t\right\| \leq \int_{s_{1}}^{s_{2}} m(t)\left\|\left(\begin{array}{c}
-0.2 \sin (t)+0.2 \\
0.4 \cos (t)+0.2
\end{array}\right)\right\| d t \\
& \leq \int_{s_{1}}^{s_{2}} m(t) \max \{|-0.2 \sin (t)+0.2|,|0.4 \cos (t)+0.2|\} d t \\
& \leq \int_{s_{1}}^{s_{2}} m(t) 0.6 d t .
\end{aligned}
$$

Therefore, if we define $Q(t)=0.6$, for all $t \in \mathbb{R}$, then condition (d) holds.

Now, let us verify that condition (e) holds. Let $m: \mathbb{R} \rightarrow \mathbb{R}_{+}$be a non-negative function and define the functions $L(t)=E_{1}(t)=0.005$ and $V(t)=E_{2}(t)=0.01$, for all $t \in \mathbb{R}$. Then, for all $s_{1}, s_{2} \in[0,2 \pi], s_{1} \leq s_{2}$, we have

$$
\begin{aligned}
& \left\|\int_{s_{1}}^{s_{2}} m(t) A(t) h(x(t-\tau(t))) d t\right\| \leq \\
& \leq \int_{s_{1}}^{s_{2}} m(t)\|A(t)\|\left\|\left(\frac{\left|x_{1}(t-\tau(t))+1\right|}{\frac{\left|x_{2}(t-\tau(t))-1\right|}{2}}\right)\right\| d t \\
& \leq \int_{s_{1}}^{s_{2}} m(t) 0.005 \max \left\{\frac{\left|x_{1}(t-\tau(t))+1\right|}{2}, \frac{\left|x_{2}(t-\tau(t))-1\right|}{2}\right\} d t \\
& \leq \int_{s_{1}}^{s_{2}} m(t) 0.005 \frac{1}{2}\left(\left|x_{1}(t-\tau(t))+1\right|+\left|x_{2}(t-\tau(t))-1\right|\right) d t
\end{aligned}
$$




$$
\begin{aligned}
& \leq \int_{s_{1}}^{s_{2}} m(t) 0.005 \frac{1}{2}\left(\left|x_{1}(t-\tau(t))\right|+\left|x_{2}(t-\tau(t))\right|+2\right) d t \\
& \leq \int_{s_{1}}^{s_{2}} m(t) 0.005 \frac{1}{2}\left(2|x|_{2 \pi}+2\right) d t \leq \int_{s_{1}}^{s_{2}} m(t) 0.005\left(|x|_{2 \pi}+1\right) d t \\
& \leq \int_{s_{1}}^{s_{2}} m(t)\left[L(t)|x|_{2 \pi}+E_{1}(t)\right] d t,
\end{aligned}
$$

and also,

$$
\begin{aligned}
& \left\|\int_{s_{1}}^{s_{2}} m(t)\left(\int_{-\infty}^{t} K(t-s) g(x(s)) d s\right) d t\right\|= \\
& =\left\|\int_{s_{1}}^{s_{2}} m(t)\left(\int_{-\infty}^{t}\left(\begin{array}{cc}
0.01 e^{-t+s} & 0 \\
0 & 0.01 e^{-t+s}
\end{array}\right)\left(\begin{array}{c}
\frac{\left|x_{1}(s)+1\right|}{2} \\
\frac{\left|x_{2}(s)-1\right|}{2}
\end{array}\right) d s\right) d t\right\| \\
& \leq \int_{s_{1}}^{s_{2}} m(t)\left(\int_{-\infty}^{t} 0.01 e^{-t+s}\left\|\left(\begin{array}{c}
\frac{\left|x_{1}(s)+1\right|}{2} \\
\frac{\left|x_{2}(s)-1\right|}{2}
\end{array}\right)\right\| d s\right) d t \\
& \leq \int_{s_{1}}^{s_{2}} m(t)\left(\int_{-\infty}^{t} 0.01 e^{-t+s}\left\|\left(\begin{array}{c}
\frac{\left|x_{1}(s)+1\right|}{2} \\
\frac{\left.\mid x_{2}(s)\right)-1 \mid}{2}
\end{array}\right)\right\| d s\right) d t \\
& \leq \int_{s_{1}}^{s_{2}} m(t)\left(\int_{-\infty}^{t} 0.01 e^{-t+s} \frac{1}{2}\left(\left|x_{1}(s)+1\right|+\left|x_{2}(s)-1\right|\right) d s\right) d t \\
& \leq \int_{s_{1}}^{s_{2}} m(t)\left(\int_{-\infty}^{t} 0.01 e^{-t+s}\left(|x|_{2 \pi}+1\right) d s\right) d t \\
& \leq \int_{s_{1}}^{s_{2}} m(t) 0.01\left(|x|_{2 \pi}+1\right) d t \\
& \leq \int_{s_{1}}^{s_{2}} m(t)\left[V(t)|x|_{2 \pi}+E_{2}(t)\right] d t .
\end{aligned}
$$

Let us verify the validity of condition (f). Let $\ell: \mathbb{R} \rightarrow \mathbb{R}_{+}$be a non-negative function and define the functions $M(t)=0.003, O(t)=0.0025$ and $N(t)=0.005$, for all $t \in \mathbb{R}$. Then, for all $s_{1}, s_{2} \in[0,2 \pi], s_{1} \leq s_{2}$,

$$
\begin{gathered}
\left\|\int_{s_{1}}^{s_{2}} \ell(t) C(t)[x(t-\sigma(t))-y(t-\sigma(t))] d t\right\| \leq \\
\leq \int_{s_{1}}^{s_{2}} \ell(t)\|C(t)\||x-y|_{2 \pi} d t \\
\quad \leq \int_{s_{1}}^{s_{2}} \ell(t) 0.003|x-y|_{2 \pi} d t \\
\quad \leq \int_{s_{1}}^{s_{2}} \ell(t) M(t)|x-y|_{2 \pi} d t \\
\left\|\int_{s_{1}}^{s_{2}} \ell(t) A(t)[h(x(t-\tau(t)))-h(y(t-\tau(t)))] d t\right\| \leq \\
\leq \int_{s_{1}}^{s_{2}} \ell(t)\|A(t)\|\left\|\left(\begin{array}{l}
\frac{\left|x_{1}(t-\tau(t))+1\right|}{2}-\frac{\left|y_{1}(t-\tau(t))+1\right|}{2} \\
2
\end{array}\right)\right\| d t
\end{gathered}
$$




$$
\begin{aligned}
& \leq \int_{s_{1}}^{s_{2}} \ell(t) 0.005 \max \left\{\frac{\left|x_{1}(t-\tau(t))-y_{1}(t-\tau(t))\right|}{2}, \frac{\left|x_{2}(t-\tau(t))-y_{2}(t-\tau(t))\right|}{2}\right\} d t \\
& \leq \int_{s_{1}}^{s_{2}} \ell(t) \frac{0.005}{2} \max \left\{\left|x_{1}(t-\tau(t))-y_{1}(t-\tau(t))\right|,\left|x_{2}(t-\tau(t))-y_{2}(t-\tau(t))\right|\right\} d t \\
& =\int_{s_{1}}^{s_{2}} \ell(t) 0.0025\|x(t-\tau(t))-y(t-\tau(t))\| d t \\
& \leq \int_{s_{1}}^{s_{2}} \ell(t) O(t)|x-y|_{2 \pi} d t,
\end{aligned}
$$

and, finally,

$$
\begin{aligned}
& \left\|\int_{s_{1}}^{s_{2}} \ell(t)\left(\int_{-\infty}^{t} K(t-s)[g(x(s))-g(y(s))] d s\right) d t\right\| \leq \\
& \leq \int_{s_{1}}^{s_{2}} \ell(t)\left(\int_{-\infty}^{t} 0.01 e^{-t+s}\left\|\left(\frac{\left|x_{1}(s)+1\right|}{2}-\frac{\left|y_{1}(s)+1\right|}{2}\right)\right\| d s\right) d t \\
& \leq \int_{s_{1}}^{s_{2}} \ell(t)\left(\int_{-\infty}^{t} \frac{0.01}{2} e^{-t+s} \max \left\{\left|x_{1}(s)-y_{1}(s)\right|,\left|x_{2}(s)-y_{2}(s)\right|\right\} d s\right) d t \\
& \leq \int_{s_{1}}^{s_{2}} \ell(t) 0.005|x-y|_{2 \pi} d t \\
& \leq \int_{s_{1}}^{s_{2}} \ell(t) N(t)|x-y|_{2 \pi} d t .
\end{aligned}
$$

Lastly, condition $(\mathrm{g})$ is true since

$$
\int_{0}^{2 \pi} \frac{D}{d}[M(s)+L(s)+V(s)] d s \leq 0.12 .
$$

Now, since $\frac{2 \pi D}{d} \sup _{t \in[0,2 \pi]}[M(t)+O(t)+N(t)]<\frac{1}{2}$, it follows by Example 6.2.2 that if

$$
\beta \geq \frac{4 T}{d} \sup _{t \in[0, T]}\left[E_{1}(t)+E_{2}(t)+Q(t)\right]=\frac{4(2 \pi)}{0,95}[0.005+0.001+0.6]=16.3,
$$

then there exists a unique $2 \pi$-periodic solution for the $2 D$-neural networks equation (6.7), under the conditions presented at the beginning of this example, in $U_{\beta}=\left\{x \in \mathscr{F}_{2 \pi}\left(\mathbb{R}, \mathbb{R}^{2}\right):|x|_{2 \pi} \leq \beta\right\}$. 


\section{BIBLIOGRAPHY}

AFONSO, S. M.; BONOTTO, E. M.; SILVA, M. R. Periodic solutions of measure functional differential equations. Citations on pages 11 and 13.

. Periodic solutions of neutral functional differential equations. Citations on pages 11 and 13.

AGARWAL, R. P.; MEEHAN, M.; O'REGAN, D. Fixed Point Theory and Applications. Cambridge: Cambridge University Press, 2001. (Cambridge Tracts in Mathematics). Citation on page 30 .

AOUITI, C.; M'HAMDI, M. S.; CHÉRIF, F. New results for impulsive recurrent neural networks with time-varying coefficients and mixed delays. Neural Process Lett, v. 46, n. 1, p. 487-506, 2017. Citation on page 104.

AP.SILVA, M. Teoria de oscilações para EDOs generalizadas e aplicações a outros tipos de equações. Phd Thesis (PhD Thesis) — Universidade de São Paulo-ICMC, São Carlos, Brazil, 2021. Citation on page 20.

ARINO, O.; HBID, M. L.; DADS, E. A. Delay Differential Equations and Applications. 1. ed. Marrakech, Morocco: Springer Netherlands, 2002. x+570 p. (NATO Science Series II: Mathematics, Physics and Chemistry, v. 205). ISBN 978-1-4020-3645-3. Citation on page 17.

BACAER, N.; ABDURAHMAN, X. Resonance of the epidemic threshold in a periodic environment. Journal of Mathematical Biology, v. 57, n. 5, p. 649-673, 2008. Citation on page 21.

BARTLE, R. A Modern Theory of Integration. Providence, Rhode Island: American Mathematical Society, 2001. (Graduate studies in mathematics). ISBN 9780821883853. Available: $<$ https://books.google.com.br/books?id=6-9royzoBL8C $>$. Citations on pages 33, 35, 36, 38, 39, 40 , and 41.

BONOTTO, E. D. M.; FEDERSON, M.; MESQUITA, J. Generalized Ordinary Differential Equations in Abstract Spaces and Applications. Hoboken, NJ: John Wiley \& Sons, Inc., 2021. ISBN 9781119654933. Available: <https://books.google.com.br/books?id=PNw9EAAAQBAJ>. Citations on pages 32, 33, 34, 35, 36, 38, and 41 .

BURTON, P. W. E. T. A.; ISLAM., M. N. Nonlinear integrodifferential equations and a priori bounds on periodic solutions. Annali di Matematica Pura ed Applicata, v. 161, p. 271-283, 1992. Available: <https://doi.org/10.1007/BF01759641>. Citation on page 20.

BURTON, T. Fixed points and differential equations with asymptotically constant or periodic solutions. Electronic Journal of Qualitative Theory of Differential Equations, v. 11, p. 1-31, 2004. ISSN 1417-3875. Available: <http://real.mtak.hu/id/eprint/22733>. Citation on page 18.

BURTON, T. A.; ZHANG., B. Periodic solutions of abstract differential equations with infinite delay. Journal of Differential Equations, Academic Press, Inc., v. 90, p. 357-396, 1991. Available: <https://doi.org/10.1016/0022-0396(91)90153-Z>. Citation on page 20. 
CHIU, K. S.; JIMÉNEZ, M. P. Periodic solutions of differential equations with a general piecewise constant argument and applications. Electronic Journal of Qualitative Theory of Differential Equations, v. 46, p. 1-19, 2010. Citation on page 21.

COOKE, K.; KAPLAN, J. A periodicity threshold theorem for epidemics and population growth. Bellman Prize in Mathematical Biosciences, v. 31, p. 87-104, 1976. Citation on page 18.

COOKE, K.; YORKE, J. Some equations modelling growth processes and gonorrhea epidemics. Bellman Prize in Mathematical Biosciences, v. 16, p. 75-101, 1973. Citation on page 18.

DAS, P. C.; SHARMA, R. R. On optimal controls for measure delay-differential equations. SIAM Journal on Control, v. 9, p. 43-61, 1971. Citation on page 19.

Existence and stability of measure differential equations. Czechoslovak Mathematical Journal, Institute of Mathematics, Academy of Sciences of the Czech Republic, v. 22, n. 1, p. 145-158, 1972. Available: <http://eudml.org/doc/12643>. Citation on page 19.

DIB, Y. M.; MAROUN, M. R.; RAFFOUL, Y. N. Periodicity and stability of neutral nonlinear system of differential equations with functional delay. Electronic Journal of Differential Equations, v. 2005, n. 142, p. 1-11, 2005. ISSN 1072-6691. Available: <http://ejde.math.txstate. edu0>. Citation on page 18 .

DONG, T.; HU, W.; ZHAO, H. Dynamic analysis for a kaldor-kalecki model of business cycle with time delay and diffusion effect. Complexity, 2018. Citation on page 21.

FARIA, T.; GADOTTI, M.; OLIVEIRA, J. Stability results for impulsive functional differential equations with infinite delay. Nonlinear Analysis: Theory, Methods \& Applications, v. 75, p. 6570-6587, 12 2012. Citation on page 21.

FEDERSON, M.; MESQUITA, J. G.; SLAVÍK, A. Measure functional differential equations and functional dynamic equations on time scales. Journal of Differential Equations, v. 252, n. 6, p. 3816-3847, 2012. ISSN 0022-0396. Available: <https://doi.org/10.1016/j.jde.2011.11.005>. Citation on page 19 .

FRANKOVÁ, D. Regulated functions. Mathematica Bohemica, Institute of Mathematics, Academy of Sciences of the Czech Republic, v. 116, n. 1, p. 20-59, 1991. Available: <http: //eudml.org/doc/29349>. Citation on page 32.

Regulated functions with values in Banach space. Mathematica Bohemica, Institute of Mathematics, Academy of Sciences of the Czech Republic, v. 144, n. 4, p. 437-456, 2019. Available: <http://eudml.org/doc/294311>. Citation on page 32.

FURTADO, A. Sobre soluções periódicas de equações diferenciais com retardos e impulsos. Phd Thesis (PhD Thesis) — Universidade de São Paulo-ICMC, São Carlos, Brazil, 2012. Citation on page 22 .

GOPALSAMY, K. Stability and Oscillations in Delay Differential Equations of Population Dynamics. Dordrecht: Springer Netherlands, 1992. (Mathematics and Its Applications). ISBN 9780792315940. Available: <https://books.google.com.br/books?id=BXbK_T_PSdwC>. Citation on page 18.

GOPALSAMY, K.; HE, X. Z.; WEN, L. Z. On a periodic neutral logistic equation. Glasgow Mathematical Journal, v. 33, n. 3, p. 281-286, 1991. ISSN 0017-0895. Available: <https: //doi.org/10.1017/S001708950000834X>. Citation on page 18. 
GRANAS, A.; DUGUNDJI, J. Fixed Point Theory. Springer New York, 2013. (Springer Monographs in Mathematics). ISBN 9780387215938. Available: <https://books.google.pn/ books?id=apLzBwAAQBAJ>. Citations on pages 25, 26, 28, and 30.

HALE, J. Theory of functional differential equations. Second. New York-Heidelberg: SpringerVerlag, 1977. x+365 p. Applied Mathematical Sciences, Vol. 3. Citation on page 18.

HENSTOCK, R. The general theory of integration. New York: Clarendon Press, 1991. (Oxford mathematical monographs). ISBN 9780198535669. Available: <https://books.google.com.br/ books?id=ETTvAAAAMAAJ>. Citation on page 33 .

HÖNIG, C. S. Volterra Stieltjes-integral equations. Amsterdam: North-Holland Publishing Co., 1975 . x+157 p. Functional analytic methods; linear constraints, Mathematics Studies, No. 16, Notas de Matemática, No. 56. [Notes on Mathematics, No. 56]. Citation on page 31.

ISLAM, M. N.; RAFFOUL, Y. N. Periodic solutions of neutral nonlinear system of differential equations with functional delay. Journal of Mathematical Analysis and Applications, v. 331, n. 2, p. 1175-1186, 2007. ISSN 0022-247X. Available: <https://doi.org/10.1016/j.jmaa.2006.09. 030>. Citations on pages 18 and 22.

ISRAELSSON, D.; JOHNSSON, A. A theory for circumnutations in helianthus annuus. Physiologia Plantarum, v. 20, p. 957-976, 1967. Citation on page 17.

KAUFMAN, C. G.; VENTURA, V.; KASS, R. E. Spline-based non-parametric regression for periodic functions and its application to directional tuning of neurons. Statistics in medicine, v. 24, n. 14, p. 2255-2265, 2005. Citation on page 21.

$\mathrm{KE}, \mathrm{W}$. On the existence and uniqueness of periodic solutions of volterra integral differential equations. Journal of Systems Science and Complexity, v. 21, n. sup1, p. 21-29, 1990. Citations on pages 18 and 22.

KRISZTIN, T.; WU, J. Asymptotic periodicity, monotonicity, and oscillation of solutions of scalar neutral functional differential equations. Journal of Mathematical Analysis and Applications, v. 199 , p. 502-525, 1996. Citation on page 18.

KURTZ, D.; SWARTZ, C. Theories of Integration: The Integrals of Riemann, Lebesgue, Henstock-Kurzweil, and McShane. River Edge, NJ: World Scientific Publishing Company, 2004. $x+283$ p. (Series in Real Analysis, v. 9). ISBN 981-238-843-5. Citation on page 41.

LI, M.; KOU, C.; DUAN, Y. The existence of periodic solution of impulsive functional differential equations with infinite delay. Journal of Applied Mathematics and Computing, v. 29, n. 1, p. 341 - 348, Jan 2009. ISSN 1865-2085. Available: <https://doi.org/10.1007/s12190-008-0134-4>. Citations on pages 22 and 97.

LIU, X.; TAKEUCHI, Y. Periodicity and global dynamics of an impulsive delay lasota-wazewska model. Journal of Mathematical Analysis and Applications, v. 327, n. 1, p. 326-341, 2007. ISSN 0022-247X. Available: <https://www.sciencedirect.com/science/article/ pii/S0022247X06003957>. Citation on page 18.

MESQUITA, C. Applications of topological degree theory to Generalized ODEs. Phd Thesis (PhD Thesis) - Universidade Federal de São Carlos, São Carlos, Brazil, 2020. Citation on page 20 . 
O'REGAN, D. Fixed-point theory for the sum of two operators. Applied Mathematics Letters, v. 9, n. 1, p. 1-8, 1996. ISSN 0893-9659. Available: <https://www.sciencedirect.com/science/ article/pii/0893965995000933>. Citations on pages 28, 30, and 75 .

PANDIT, S. G.; DEO, S. G. Differential systems involving impulses. Berlin: Springer-Verlag, 1982. vii+102 p. (Lecture Notes in Mathematics, v. 954). ISBN 3-540-11606-0. Citation on page 19.

SCHMAEDEKE, W. W. Optimal control theory for nonlinear vector differential equations containing measures. SIAM Journal on Control, v. 3, p. 231-280, 1965. Citation on page 19.

SCHWABIK, Š. Generalized ordinary differential equations. River Edge, NJ: World Scientific Publishing Co. Inc., 1992. x+382 p. (Series in Real Analysis, v. 5). ISBN 981-02-1225-9. Citations on pages 19, 33, 34, and 35 .

Abstract Perron-Stieltjes integral. Mathematica Bohemica, Institute of Mathematics, Academy of Sciences of the Czech Republic, v. 121, n. 4, p. 425-447, 1996. Available: <http: //eudml.org/doc/247962>. Citation on page 33.

SHARMA, R. R. An abstract measure differential equation. Proceedings of the American Mathematical Society, v. 32, p. 503-510, 1972. ISSN 0002-9939. Citation on page 19.

SOMOLINOS, A. S. Periodic solutions of the sunflower equation: $x^{\prime \prime}+(a / r) x^{\prime}+(b / r) \operatorname{sen}(x(t-$ $r))=0$. Quarterly of Applied Mathematics, p. 465-478, 1978. Citation on page 17.

STAMOVA, I.; STAMOV, G. Applied Impulsive Mathematical Models. Cham: Springer International Publishing, 2016. (1613-5237). ISBN 978-3-319-28061-5. Citations on pages 18, 101 , and 104.

STAMOVA, I. M.; STAMOV, G. T. Functional and Impulsive Differential Equations of Fractional Order. Boca Raton, FL: CRC Press, 2021. ISBN 99780367782726. Citation on page 21 .

WU, J.; XIA, H.; ZHANG, B. Topological transversality and periodic solutions of neutral functional differential equations. Proceedings of the Royal Society of Edinburgh: Section A Mathematics, Royal Society of Edinburgh Scotland Foundation, v. 129, n. 1, p. 199-220, 1999. Citations on pages 20, 22, and 28.

YAN, J.; ZHAO, A. Oscillation and stability of linear impulsive delay differential equations. Journal of Mathematical Analysis and Applications, v. 227, n. 1, p. 187-194, 1998. ISSN 0022247X. Available: <http://www.sciencedirect.com/science/article/pii/S0022247X98960930>. Citations on pages 22 and 97.

YEE, L.; VỲBORNỲ, R.; VYBORNY, R.; SOCIETY, A. M.; BROADBRIDGE, P.; HEYDE, C.; MURRAY, M. Integral: An Easy Approach After Kurzweil and Henstock. Cambridge, UK: Cambridge University Press, 2000. (Australian Mathematical Society Lecture Series). ISBN 9780521779685. Available: <https://books.google.com.br/books?id=yJEtjnykjYEC>. Citations on pages $33,34,35,36$, and 41 .

ZEIDLER, E. Nonlinear Functional Analysis and its Applications. River Edge, NJ: SpringerVerlag New York., 1986. x+909 p. (Springer-Verlag New York, Inc., v. 1). ISBN 978-0-38790914-1. Citation on page 29. 
ZHAO, Y.; FENG, Z.; DING, W. Existence and stability of periodic solution of impulsive neural systems with complex deviating arguments. Journal of Biological Dynamics, Taylor and Francis, v. 9, n. sup1, p. 291-306, 2015. PMID: 25397685. Citations on pages 22 and 97. 


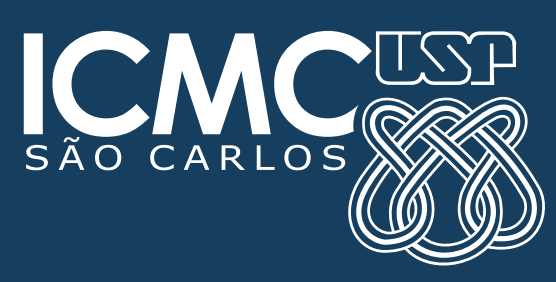

